\title{
Central values of $L$-functions of cubic twists
}

\author{
Eugenia Rosu ${ }^{1,2}$
}

Received: 23 April 2019 / Revised: 3 February 2020 / Published online: 25 August 2020

(c) The Author(s) 2020

\section{Abstract}

We are interested in finding for which positive integers $D$ we have rational solutions for the equation $x^{3}+y^{3}=D$. The aim of this paper is to compute the value of the $L$-function $L\left(E_{D}, 1\right)$ for the elliptic curves $E_{D}: x^{3}+y^{3}=D$. For the case of $p$ prime $p \equiv 1 \bmod 9$, two formulas have been computed by Rodriguez-Villegas and Zagier. We have computed formulas that relate $L\left(E_{D}, 1\right)$ to the square of a trace of a modular function at a CM point. This offers a criterion for when the integer $D$ is the sum of two rational cubes. Furthermore, when $L\left(E_{D}, 1\right)$ is nonzero we get a formula for the number of elements in the Tate-Shafarevich group and we show that this number is a square when $D$ is a norm in $\mathbb{Q}[\sqrt{-3}]$.

Mathematics Subject Classification Primary 11G40 - 11F67; Secondary 14H52

\section{Introduction}

In the current paper we are interested in finding which positive integers $D$ can be written as the sum of two rational cubes:

$$
x^{3}+y^{3}=D, \quad x, y \in \mathbb{Q} .
$$

Despite the simplicity of the problem, an elementary approach to solving the Diophantine equation fails. However, we can restate the problem in the language of elliptic curves. After making the equation homogeneous, we get the equation $x^{3}+y^{3}=D z^{3}$ that has a rational point at $\infty=[1:-1: 0]$. Moreover, after a change of coordinates

\section{Communicated by Wei Zhang.}

$凶 \quad$ Eugenia Rosu

rosu@math.arizona.edu

1 Max Planck Institute for Mathematics, Bonn, Germany

2 Department of Mathematics, University of Arizona, Tucson, AZ 85721, USA 
$X=12 D \frac{z}{x+y}, Y=36 D \frac{x-y}{x+y}$ the equation becomes:

$$
E_{D}: Y^{2}=X^{3}-432 D^{2}
$$

which defines an elliptic curve over $\mathbb{Q}$ written in its Weierstrass affine form.

Thus the problem reduces to finding if the group of rational points $E_{D}(\mathbb{Q})$ of the elliptic curve $E_{D}$ is non-trivial. We assume $D$ cube free and $D \neq 1,2$ throughout the paper. In this case $E_{D}(\mathbb{Q})$ has trivial torsion (see [27]), thus (1) has a solution iff $E_{D}(\mathbb{Q})$ has positive rank. From the Birch and Swinnerton-Dyer $(B S D)$ conjecture, this is conjecturally equivalent to the vanishing of $L\left(E_{D}, 1\right)$.

Without assuming BSD, from the work of Coates-Wiles [3] (or more generally Gross-Zagier [9] and Kolyvagin [17]), when $L\left(E_{D}, 1\right) \neq 0$ the rank of $E_{D}(\mathbb{Q})$ is 0 , thus we have no rational solutions in (1).

In the case of prime numbers, Sylvester conjectured that we have solutions in (1) in the case of $D \equiv 4,7,8 \bmod 9$. In the cases of $D$ prime with $D \equiv 2,5 \bmod 9$, $D$ is not the sum of two cubes. This follows from a 3-descent argument (given in the 19 th century by Sylvester, Lucas and Pepin).

We define the invariant

$$
S_{D}=\frac{L\left(E_{D}, 1\right)}{c_{3 D} \Omega_{D}},
$$

where $\Omega_{D}=\frac{\sqrt{3}}{6 \pi \sqrt[3]{D}} \Gamma\left(\frac{1}{3}\right)^{3}$ is the real period and $c_{3 D}=\prod_{p \mid 3 D} c_{p}$ is the product of the Tamagawa numbers $c_{p}$ corresponding to the elliptic curve $E_{D}$ at the unramified places $p \mid 3 D$. The definition is made such that in the case of $L\left(E_{D}, 1\right) \neq 0$ we expect to get from the full BSD conjecture:

$$
S_{D}=\# \amalg\left(E_{D}\right),
$$

where \#Ш is the order of the Tate-Shafarevich group.

From the work of Rubin [25], $L\left(E_{D}, 1\right) \neq 0$ implies the order of $\amalg\left(E_{D}\right)$ is finite. Furthermore, using the Cassels-Tate pairing, Cassels proved in [2] that when $\amalg$ is finite the order \#W is a square. We actually show that, when $D$ is a norm in $\mathbb{Q}[\sqrt{-3}], S_{D}$ is an integer square up to an even power of 3 . Current work in Iwasawa theory shows that for semistable elliptic curves at the good primes $p$ we have $\operatorname{ord}_{p}\left(\# \amalg\left[p^{\infty}\right]\right)=\operatorname{ord}_{p}\left(S_{D}\right)$, where $\amalg\left[p^{\infty}\right]$ is the $p^{\infty}$-torsion part of $\amalg$ (see [14]). However, this cannot be applied at the place 3 in our case, as this is a prime of bad reduction for $E_{D}$.

By computing the value of $S_{D}$, one can determine when we have solutions in (1) and, assuming the full BSD conjecture, one can find in certain cases the order of $\amalg$ :

(i) $S_{D} \neq 0 \Longrightarrow$ no solutions in (1)

(ii) $S_{D} \neq 0 \stackrel{B S D}{\Longrightarrow} S_{D}=\# \amalg$ integer square

(iii) $S_{D}=0 \stackrel{B S D}{\Longrightarrow}$ have solutions in (1). 
The goal of the current paper is to compute several formulas for $S_{D}$. In [24], Rodriguez-Villegas and Zagier computed formulas for $L\left(E_{p}, 1\right)$ in the case of primes $p \equiv 1 \bmod 9$. In the current paper we are extending on their results and compute similar formulas for all integers $D$.

Our main theorem is the following:

Theorem 1 For $D=\prod_{p_{i} \equiv 1(3)} p_{i}^{e_{i}}, S_{D}$ is an integer square up to an even power of 3.

Theorem 1 above follows from the formula for $S_{D}$ presented below. Let $K=$ $\mathbb{Q}[\sqrt{-3}]$. For $D$ a norm in $K$, we write $D=D_{1} D_{2}^{2}$ such that $D_{0}=D_{1} D_{2}$ is the radical of $D$. Let $\pi_{1}, \pi_{2} \equiv 1 \bmod 3$ be elements of norm $D_{1}$ and $D_{2}$ respectively. Let $\sigma(D)$ the number of distinct primes dividing $D$ and $\varphi$ Euler's totient function.

Theorem 2 Using the above notation, let $D=\prod_{p_{i} \equiv 1(3)} p_{i}^{e_{i}}$ be a positive integer that is a product of split primes in $K$ and $D_{0}=\prod_{p \mid D} p$ be its radical. Then $S_{D}$ is an integer square up to an even power of 3 and we have:

$$
S_{D}=T_{D}^{2} \frac{1}{(-3)^{2+\sigma(D)}},
$$

where the term $T_{D} / 3$ is an integer if $\sigma(D)$ is even and $T_{D} / \sqrt{-3}$ is an integer if $\sigma(D)$ is odd. Moreover, we have the formula:

$$
T_{D}=\frac{1}{\varphi\left(D_{0}\right)} \operatorname{Tr}_{H_{\mathscr{O}} / K}\left(\frac{\theta_{1}(\tau)}{\theta_{0}(\tau)} \omega^{k_{0}} \bar{\pi}_{1}^{-2 / 3} \pi_{2}^{1 / 3}\right),
$$

where:

$-\theta_{r}(z)=\sum_{n \in \mathbb{Z}}(-1)^{n} e^{\pi i\left(n+\frac{r}{D}-\frac{1}{6}\right)^{2} z}$, for $r=0,1$ are theta functions of weight $1 / 2$,

$-\tau=\frac{-b+\sqrt{-3}}{2}$ is a CM point such that $b^{2} \equiv-3 \bmod 12 D^{2}$ and $\left(\pi_{1} \pi_{2}\right)^{2}$ divides $(\tau)$

- $H_{\mathscr{O}}$ is the ray class field of modulus $3 D_{0}$,

- $\omega^{k_{0}}$ is the unique cube root of unity that makes $T_{D} / 3$ or $T_{D} / \sqrt{-3}$ an integer.

This theorem follows from a more general result for all integers $D$ prime to 6 that is proved using automorphic methods:

Theorem 3 For all integers $D$ prime to $6,3 c_{3 D} S_{D}$ is an integer and we have the formula:

$$
S_{D}=\frac{1}{3 c_{3 D}} \operatorname{Tr}_{H_{3 D} / K}\left(D^{1 / 3} \frac{\Theta_{K}(D \omega)}{\Theta_{K}(\omega)}\right),
$$

where $\Theta_{K}(z)=\sum_{a, b \in \mathbb{Z}} e^{2 \pi i z\left(a^{2}+b^{2}-a b\right)}$ is the theta function of weight one associated to the number field $K, \omega=\frac{-1+\sqrt{-3}}{2}$ is a third root of unity, and $H_{3 D}$ is the ring class field associated to the order $\mathscr{O}_{3 D}=\mathbb{Z}+3 D \mathscr{O}_{K}$. 
Note that each of the elliptic curves $E_{D}$ is a cubic twist of $E_{1}$. In the case of quadratic twists of elliptic curves, an important tool in computing the values of the $L$-functions is the work of Waldspurger [32]. For example, this is used to obtain Tunnell's theorem for congruent numbers in [31]. However, the cubic twist case proves to be significantly more difficult. We instead take advantage of the fact that $E_{D}$ is an elliptic curve with complex multiplication by $\mathscr{O}_{K}=\mathbb{Z}[\omega]$ the ring of integers of the number field $K$. Then from CM theory there is a Hecke character $\chi_{E_{D}}: \mathbb{A}_{K}^{\times} / K^{\times} \rightarrow \mathbb{C}^{\times}$such that $L\left(E_{D}, s\right)=L\left(s, \chi_{E_{D}}\right)$ and we compute the value of $L\left(s, \chi_{E_{D}}\right)$ using automorphic methods.

We present now an outline of the proof of Theorem 3. To compute the value of $L\left(s, \chi_{E_{D}}\right)$ we look at the Hecke character adelically and using Tate's thesis we compute Tate's zeta function $Z\left(s, \chi_{E_{D}}, \Phi_{K}\right)$ for $\Phi_{K}$ a Schwartz-Bruhat function in $\mathscr{S}\left(\mathbb{A}_{K}\right)$. After integrating we get a linear combination of Eisenstein series. By evaluation at $s=1$, we write $L\left(E_{D}, 1\right)$ as a linear combination of theta functions at CM-points. We further show using Shimura's reciprocity law that the terms are all Galois conjugates over $K$.

The idea of the proof of Theorem 2 is based on factoring each weight one theta function $\Theta_{K}(z)$ into a product of theta functions of weight $1 / 2$. The method we are using is a factorization lemma of Rodriguez-Villegas and Zagier from [23] applied to the formula in Theorem 3. This gives us the square of a linear combination of theta functions evaluated at CM points. Finally, using Shimura reciprocity law, we show that all the factors are Galois conjugates to each other and recover an integer square.

Note that using the formula (4) we can show that an integer $D$ cannot be written as the sum of two cubes by computationally checking whether $L\left(E_{D}, 1\right) \neq 0$. Furthermore, assuming BSD, $S_{D}=\# \amalg$ and thus we can compute the expected order of $\amalg$ explicitly. We provide below some examples when $S_{D} \neq 0$ that we computed using PARI:

\begin{tabular}{llllll}
\hline $\mathrm{D}$ & \#Ш & $\mathrm{D}$ & \#Ш & $\mathrm{D}$ & \#Ш \\
\hline $19 \cdot 37$ & 1 & $37 \cdot 73$ & 1 & $13 \cdot 19 \cdot 79$ & 1 \\
$31 \cdot 43$ & 1 & $43 \cdot 67$ & 1 & $13 \cdot 19 \cdot 61$ & 1 \\
$31 \cdot 79$ & 1 & $43 \cdot 193$ & 4 & $13 \cdot 37 \cdot 61$ & 1 \\
$31 \cdot 97$ & 1 & $61 \cdot 67$ & 1 & $13 \cdot 43 \cdot 73$ & 1 \\
$61 \cdot 103$ & 1 & $61 \cdot 139$ & 1 & $31 \cdot 37 \cdot 43$ & 1 \\
\hline
\end{tabular}

\section{Background}

Let $K=\mathbb{Q}[\sqrt{-3}]$ and denote $\mathscr{O}_{K}=\mathbb{Z}[\omega]$ its ring of integers, where $\omega=\frac{-1+\sqrt{-3}}{2}$ is a fixed cube root of unity. We will denote by $K_{v}$ the completion of $K$ at the place $v, \mathscr{O}_{K_{v}}$ the ring of integers of $K_{v}$, and for a prime $p$ in $\mathbb{Z}$ we define $K_{p}=K \otimes_{\mathbb{Q}} \mathbb{Q}_{p}$ and $\mathscr{O}_{K_{p}}=\mathscr{O}_{K} \otimes_{\mathbb{Z}} \mathbb{Z}_{p}$ the semilocal ring of integers. Note that $K_{p}=\prod_{v \mid p} K_{v} \cong$ $\mathbb{Q}_{p}[x] /\left(x^{2}-3\right)$ and $\mathscr{O}_{K_{p}} \cong \prod_{v \mid p} \mathscr{O}_{K_{v}}$. 


\subsection{The $L$-function}

Our goal is to compute several formulas for the central value of the $L$-function $L\left(E_{D}, 1\right)$ of the elliptic curve $E_{D}: x^{3}+y^{3}=D z^{3}$. The elliptic curve $E_{D}$ has complex multiplication $(\mathrm{CM})$ by $\mathscr{O}_{K}$. Then from CM theory (see [27, Chapter II]) we can find a Hecke character $\chi: \mathbb{A}_{K}^{\times} / K^{\times} \rightarrow \mathbb{C}$ corresponding to the elliptic curve $E_{D}$ such that $L\left(E_{D}, s\right)=L(s, \chi)$. We can compute explicitly $\chi=\chi_{D} \varphi$ (see Ireland and Rosen [13, Chapter 18], for more details), where $\varphi$ is the Hecke character of infinity type $(1,0)$ corresponding to $E_{1}$ and $\chi_{D}$ is the Hecke character corresponding to the cubic twist. More precisely, writing the characters classically, we have:

$-\varphi: I(3) \rightarrow K^{\times}$is defined on the set of ideals prime to 3 by $\varphi(\mathscr{A})=\alpha$, where $\alpha$ is the unique generator of the ideal $\mathscr{A}$ such that $\alpha \equiv 1 \bmod 3$.

$-\chi_{D}: \mathrm{Cl}\left(\mathscr{O}_{3 D}\right) \rightarrow\left\{1, \omega, \omega^{2}\right\}$ is the cubic character defined below in Sect. 2.2; it is defined over $\mathrm{Cl}\left(\mathscr{O}_{3 D}\right)$ the ring class group corresponding to the order $\mathscr{O}_{3 D}=$ $\mathbb{Z}+3 D \mathscr{O}_{K}$.

Note that, for $\operatorname{Re}(s)>1$, the $L$-function can be expanded as

$$
L\left(E_{D}, s\right)=\sum_{\substack{\alpha \in \mathscr{O}_{K} \\ \alpha \equiv 1(\bmod 3)}} \frac{\chi_{D}(\alpha) \alpha}{(\operatorname{Nm} \alpha)^{s}}
$$

\subsection{The cubic character}

We define the cubic character $\chi_{D}$ and recall some of its properties following Ireland and Rosen [13]. For $\alpha \in \mathscr{O}_{K}$ prime to 3 , we define the cubic residue character $\left(\frac{\alpha}{.}\right)_{3}$ : $I(3 \alpha) \rightarrow\left\{1, \omega, \omega^{2}\right\}$, where $I(3 \alpha)$ is the set of fractional ideals of $K$ prime to $3 \alpha$. For a prime ideal $\mathfrak{p}$ of $K$, we define $\left(\frac{\alpha}{\mathfrak{p}}\right)_{3}=\omega^{j}$, for $0 \leq j \leq 2$ such that

$$
\alpha^{(\mathrm{Nm} \mathfrak{p}-1) / 3} \equiv \omega^{j} \quad \bmod \mathfrak{p} .
$$

It is extended multiplicatively on the fractional ideals of $I(3 \alpha)$.

The character $\chi_{D}$ is defined following [13] to be:

$$
\chi_{D}(\mathscr{A})=\overline{\left(\frac{D}{\mathscr{A}}\right)_{3}} .
$$

We also define $\chi_{\pi}(\mathscr{A})=\overline{\left(\frac{\pi}{\mathscr{A}}\right)_{3}}$ where $\pi$ is a generator of an ideal of norm $D$. Note that $\overline{\chi_{\pi}(\mathscr{A})}=\chi_{\bar{\pi}}(\overline{\mathscr{A}})$.

An important result is the cubic reciprocity law (see [13] for more details):

Theorem 4 (Cubic reciprocity law) For $\pi_{1}, \pi_{2} \equiv 2 \bmod 3$ generators of the prime ideals $\mathfrak{p}_{1}, \mathfrak{p}_{2}$ prime to 3 , we have $\left(\frac{\pi_{1}}{\pi_{2}}\right)_{3}=\left(\frac{\pi_{2}}{\pi_{1}}\right)_{3}$. 
It follows immediately from the cubic reciprocity law that for $\alpha \equiv \pm 1 \bmod 3$, we have $\chi_{D}(\alpha)=\chi_{\alpha}(D)$. Also from the cubic reciprocity it follows that $\chi_{D}((\alpha))=1$ for $\alpha \equiv a \bmod 3 D$, where $a$ is an integer prime to $3 D$. Thus $\chi_{D}$ is trivial on the ideals of $P_{\mathbb{Z}, 3 D}=\{(\alpha): \alpha \in K$ such that $\alpha \equiv a \bmod 3 D$ for some integer $a$ such that $(a, 3 D)=1\}$. The ring class group of the order $\mathscr{O}_{3 D}=\mathbb{Z}+3 D \mathscr{O}_{K}$ is $\mathrm{Cl}\left(\mathscr{O}_{3 D}\right)=I(3 D) / P_{\mathbb{Z}, 3 D}$, where $I(3 D)$ is the set of fractional ideals prime to $3 D$, and thus $\chi_{D}$ is invariant on $\mathrm{Cl}\left(\mathscr{O}_{3 D}\right)$.

\subsection{Hecke characters}

A classical Hecke character $\tilde{\chi}: I(f) \rightarrow \mathbb{C}^{\times}$of conductor $f$ can be expressed on the set of principal ideals $P(f)$ prime to $f$ in the form $\tilde{\chi}((\alpha))=\tilde{\epsilon}(\alpha) \tilde{\chi}_{0}^{-1}(\alpha)$, where $\widetilde{\varepsilon}:\left(\mathscr{O}_{K} / f \mathscr{O}_{K}\right)^{\times} \rightarrow \mathbb{T}$ is a character taking values in a finite group $\mathbb{T}$ and $\tilde{\chi}_{0}$ is an infinity type continuous character, meaning that $\tilde{\chi}_{0}: \mathbb{C}^{\times} \rightarrow \mathbb{C}^{\times}$is a continuous character.

The idelic Hecke character is a continuous character $\chi: \mathbb{A}^{\times} / K^{\times} \rightarrow \mathbb{C}^{\times}$. There is a unique correspondence between the idelic and the classical Hecke characters defined as follows: at $\infty$ for $z \in \mathbb{C}$ we define $\chi_{\infty}(z)=\tilde{\chi}_{0}^{-1}(z)$ for $z \in \mathbb{C}^{\times}$and at the places $v \nmid f$ we define $\chi\left(\mathscr{O}_{v}^{\times} \varpi_{v}\right)=\tilde{\chi}\left(\mathfrak{p}_{v}\right)$, for $\varpi_{v}$ a uniformizer of $\mathscr{O}_{K_{v}}$ and $\mathfrak{p}_{v}$ the prime corresponding to the place $v$. At the places $v \mid f$, the value of $\chi_{v}$ can be determined using the Weak Approximation Theorem.

We are interested in the character $\chi=\chi_{D} \varphi$ defined before. By abuse of notation, we will use $\varphi, \chi_{D}$ both for the classical and the adelic Hecke characters.

Recall $\varphi: I(3) \rightarrow \mathbb{C}^{\times}$is the Hecke character defined by $\chi((\alpha))=\alpha$ for $\alpha \equiv 1$ $\bmod 3$. For the place $v \nmid 3$, denote by $\varpi_{v}$ a uniformizer of $\mathscr{O}_{K_{v}}$ such that $\varpi_{v} \equiv 1$ $\bmod 3$. Then for $\varphi: \mathbb{A}_{K}^{\times} \rightarrow \mathbb{C}^{\times}$, we have:

$-\varphi_{v}(p)=-p, \varphi_{v}\left(\mathscr{O}_{K_{v}}^{\times}\right)=1$, for $v=p, p \equiv 2 \bmod 3$,

$-\varphi_{v}\left(\varpi_{v}\right)=\varpi_{v}, \varphi_{v}\left(\mathscr{O}_{K_{v}}^{\times}\right)=1$, for $v \mid p, p \equiv 1 \bmod 3$,

$-\varphi_{\infty}\left(x_{\infty}\right)=x_{\infty}^{-1}$, at $v=\infty$.

Recall $\chi_{D}: I(3 D) \rightarrow\left\{1, \omega, \omega^{2}\right\}$ is the cubic character and we showed that it is well-defined on $\mathrm{Cl}\left(\mathscr{O}_{3 D}\right)$, the ring class group for the order $\mathscr{O}_{3 D}=\mathbb{Z}+3 D \mathscr{O}_{K}$. We define the character $\chi_{D}$ adelically over $K^{\times} \backslash \mathbb{A}_{K, f}^{\times} / U(3 D) \simeq \operatorname{Cl}\left(\mathscr{O}_{3 D}\right)$, where $U(3 D)=\left(1+3 \mathbb{Z}_{3}[\omega]\right) \prod_{p \mid D}\left(\mathbb{Z}+D \mathscr{O}_{K_{p}}\right)^{\times} \prod_{p \nmid 3 D}\left(\mathscr{O}_{K_{p}}\right)^{\times}$.

Note that we can rewrite $l_{f} \in \mathbb{A}_{K, f}^{\times}$in the form $l_{f}=k l_{1}$ with $k \in K^{\times}$and $l_{1} \in \prod_{v \nmid \infty} \mathscr{O}_{K_{v}}^{\times}$. We can find $k_{1} \in \mathscr{O}_{K}$ such that $k_{1} \equiv l_{1} \bmod 3 D \mathscr{O}_{K_{v}}$ and we define $\chi_{D, f}(l)=\chi_{D, f}\left(l_{1}\right)=\chi_{D}\left(\left(k_{1}\right)\right)$. More precisely, we get:

$-\chi_{D, v}\left(\varpi_{v}\right)=\chi_{D}\left(\mathfrak{p}_{v}\right)$ and $\chi_{D, v}\left(\mathscr{O}_{K_{v}}^{\times}\right)=1$, if $v \nmid 3 D$,

$-\chi_{D, \infty}\left(x_{\infty}\right)=1$, at $v=\infty$.

The values of $\chi_{D}$ and $\varphi$ at the ramified places can be computed using the Weak approximation theorem. 


\section{$3 L\left(E_{D}, 1\right)$ and Tate's zeta function}

In this section we will compute the value of $L\left(E_{D}, 1\right)=L\left(1, \chi_{D} \varphi\right)$, working with $\chi_{D}, \varphi$ as automorphic Hecke characters. Let $K=\mathbb{Q}[\sqrt{-3}]$ and $\omega=\frac{-1+\sqrt{-3}}{2}$ a fixed cube root of unity as before. We will show the following result:

Theorem 5 For $S_{D}=\frac{2 \sqrt{3} \pi D^{-1 / 3}}{c_{3 D} \Gamma\left(\frac{1}{3}\right)^{3}} L\left(E_{D}, 1\right)$, we have $3 c_{3 D} S_{D} \in \mathbb{Z}$ and

$$
S_{D}=\frac{1}{3 c_{3 D}} \operatorname{Tr}_{H_{3 D} / K}\left(D^{1 / 3} \frac{\Theta_{K}(D \omega)}{\Theta_{K}(\omega)}\right)
$$

where $\Theta_{K}(z)=\sum_{m, n \in \mathbb{Z}} e^{2 \pi i\left(m^{2}+n^{2}-m n\right) z}, H_{3 D}$ is the ring class field for the order $\mathscr{O}_{3 D}=\mathbb{Z}+3 D \mathscr{O}_{K}$ and $c_{3 D}=\prod_{p \mid 3 D} c_{p}$ is the product of the Tamagawa numbers $c_{p}$ of $E_{D}$.

We will compute the formula (5) using Tate's zeta function. We start by recalling some background and notation.

\subsection{Haar measure}

We take $V=K$ as a quadratic vector space over $\mathbb{Q}$ with the norm as its quadratic form. We take $d x_{v}$ to be the self-dual additive Haar measure and $d^{\times} \alpha_{v}$ the multiplicative Haar measure $d_{v}^{\times} x_{v}=\frac{d x_{v}}{\left|x_{v}\right|_{v}}$ normalized such that $\operatorname{vol}\left(\mathscr{O}_{K_{v}}^{\times}\right)=1$, if $v \nmid \infty$, and $d^{\times} z=\frac{d z}{|z|_{\infty}}$ where $d z$ is the usual Lebesgue measure, and $|z|_{\infty}=|z|_{\mathbb{C}}^{2}$ is the square of the usual absolute value over $\mathbb{C}$. We use the same normalization for the Haar measure as in Tate's thesis (see [1, Section 3.1]).

\subsection{Schwartz-Bruhat functions}

We choose the Schwartz-Bruhat function $\Phi_{f} \in S\left(\mathbb{A}_{K, f}\right)$ such that Tate's zeta function $Z\left(s, \Phi, \chi_{D} \varphi\right)$ defined below to be nonzero. More precisely, $\Phi_{f}=\prod_{v \nmid \infty} \Phi_{v}$, where:

$-\Phi_{v}=\operatorname{char}_{\mathscr{O}_{K v}}$ for $v \nmid 3 D$,

- $\Phi_{p}=\sum_{(a, D)=1} \operatorname{char}_{\left(a+D \mathscr{O}_{K p}\right)}$ for $p \mid D$,

$-\Phi_{v}=\operatorname{char}_{\left(1+3 \mathscr{O}_{K v}\right)}$ for $v=\sqrt{-3}$.

\subsection{Tate's zeta function}

We recall Tate's zeta function. For a Hecke character $\chi: \mathbb{A}_{K}^{\times} / K^{\times} \rightarrow \mathbb{C}^{\times}$and a Schwartz-Bruhat function $\Phi \in \mathscr{S}\left(\mathbb{A}_{K}\right)$, Tate's zeta function is defined locally as $Z_{v}\left(s, \chi_{v}, \Phi_{v}\right)=\int_{K_{v}^{\times}} \chi_{v}\left(\alpha_{v}\right)\left|\alpha_{v}\right|_{v}^{s} \Phi_{v}\left(\alpha_{v}\right) d^{\times} \alpha_{v}$, and globally as $Z(s, \chi, \Phi)=$ 
$\prod_{v} Z_{v}\left(s, \chi_{v}, \Phi_{v}\right)$. As a global integral this is

$$
Z(s, \chi, \Phi)=\int_{\mathbb{A}_{K}^{\times}} \chi(\alpha)|\alpha|^{s} \Phi(\alpha) d^{\times} \alpha
$$

It has meromorphic continuation to all $s \in \mathbb{C}$ and in our case it is entire. We will compute $Z_{f}\left(s, \chi_{f}, \Phi_{f}\right)$ for $\chi=\chi_{D} \varphi$ and the Schwartz-Bruhat function $\Phi_{f}$ chosen above.

From Tate's thesis (see [1, Proposition 3.1.4]), we have the equality of local factors $L_{v}\left(s, \chi_{D} \varphi\right)=Z_{v}\left(s, \chi_{D} \varphi\right)$ at all the unramified places, and thus $L\left(s, \chi_{D} \varphi\right)=$ $Z_{f}\left(s, \chi_{D} \varphi\right) \prod_{p \mid 3 D} \frac{L_{p}\left(s, \chi_{D, p} \varphi_{p}\right)}{Z_{p}\left(s, \chi_{D, p} \varphi_{p}, \Phi_{p}\right)}$. As $\varphi, \chi_{D}$ and $|\cdot|$ are trivial when $\Phi_{p}$ is nonzero for $p \mid 3 D$, we can compute easily

$$
\prod_{p \mid 3 D} Z_{p}\left(s, \chi_{D, p} \varphi_{p}, \Phi_{p}\right)=\prod_{p \mid D} \operatorname{vol}\left(\left(\mathbb{Z}+3 D \mathscr{O}_{K_{p}}\right)^{\times}\right) \operatorname{vol}\left(1+3 \mathbb{Z}_{3}[\omega]\right),
$$

and this equals $\frac{1}{6} \prod_{p \mid D}\left(p-\left(\frac{p}{3}\right)\right)^{-1}$. The terms $L_{p}\left(s, \chi_{D} \varphi\right)=1$ for $p \mid 3 D$ by definition. Thus for all $s$ and for $\Phi$ the Schwartz-Bruhat function chosen above, we have:

$$
L\left(s, \chi_{D} \varphi\right)=Z_{f}\left(s, \chi_{D} \varphi, \Phi\right) V_{3 D}
$$

where $V_{3 D}=\frac{1}{6} \prod_{p \mid D}\left(p-\left(\frac{p}{3}\right)\right)^{-1}$.

Next we compute the value of $Z_{f}\left(s, \chi_{D, f} \varphi_{f}, \Phi_{f}\right)$ as a linear combination of Hecke characters and use (6) to get the value of $L\left(s, \chi_{D, f} \varphi_{f}\right)$ :

Lemma 6 For all $s \in \mathbb{C}$ and the Schwartz-Bruhat function $\Phi_{f} \in \mathscr{S}\left(\mathbb{A}_{K, f}\right)$ chosen above, we have:

$$
L\left(s, \chi_{D} \varphi\right)=\sum_{\alpha_{f} \in U(3 D) \backslash \mathbb{A}_{K, f}^{\times} / K^{\times}} I\left(s, \alpha_{f}, \Phi_{f}\right) \chi_{D}(\alpha) \varphi(\alpha),
$$

where $I\left(s, \alpha_{f}, \Phi_{f}\right)=\sum_{k \in K^{\times}} \frac{k}{|k|_{\mathbb{C}}^{2 s}} \Phi_{f}\left(k \alpha_{f}\right)$ and $U(3 D)=\left(1+3 \mathbb{Z}_{3}[\omega]\right) \prod_{v \mid D}$ $\left(\mathbb{Z}+D \mathscr{O}_{K_{p}}\right)^{\times} \prod_{v \nmid 3 D} \mathscr{O}_{K_{v}}^{\times}$.

Proof We first take the quotient by $K^{\times}$in the integral defining $Z_{f}\left(s, \chi_{D} \varphi, \Phi_{f}\right)$ and get:

$$
Z_{f}\left(s, \chi_{D} \varphi, \Phi_{f}\right)=\int_{\mathbb{A}_{K, f}^{\times} / K^{\times}} \sum_{k \in K^{\times}} \chi_{D, f}\left(k \alpha_{f}^{\prime}\right) \varphi_{f}\left(k \alpha_{f}^{\prime}\right)\left|k \alpha_{f}\right|_{f}^{s} \Phi_{f}\left(k \alpha_{f}^{\prime}\right) d^{\times} \alpha_{f}^{\prime}
$$

We have $\chi_{D, f}\left(k \alpha_{f}^{\prime}\right)=\chi_{D, \infty}^{-1}(k) \chi_{D, f}\left(\alpha_{f}^{\prime}\right)=\chi_{D, f}\left(\alpha_{f}^{\prime}\right), \varphi_{f}\left(k \alpha_{f}^{\prime}\right)=\varphi_{\infty}^{-1}(k) \varphi_{f}\left(\alpha_{f}^{\prime}\right)=$ $k \varphi_{f}\left(\alpha_{f}^{\prime}\right)$ and $\left|k \alpha_{f}^{\prime}\right|_{f}^{s}=|k|_{\infty}^{-s}\left|\alpha_{f}\right|_{f}^{s}=|k|_{\mathbb{C}}^{-2 s}\left|\alpha_{f}^{\prime}\right|_{f}^{s}$, where $|\cdot| \mathbb{C}$ is the usual absolute 
value over $\mathbb{C}$. Then the integral reduces to:

$Z_{f}\left(s, \chi_{D} \varphi, \Phi_{f}\right)=\int_{\mathbb{A}_{K, f}^{\times} / K^{\times}}\left(\sum_{k \in K^{\times}} \frac{k}{|k|_{\mathbb{C}}^{2 s}} \chi_{D, f}\left(\alpha_{f}^{\prime}\right) \Phi_{f}\left(k \alpha_{f}^{\prime}\right)\right) \varphi_{f}\left(\alpha_{f}^{\prime}\right)\left|\alpha_{f}^{\prime}\right|_{f}^{s} d^{\times} \alpha_{f}^{\prime}$

Furthermore, our Schwartz-Bruhat functions $\Phi_{f}\left(k \alpha_{f}^{\prime}\right)$ are invariant on $U(3 D)$. Similarly, $|\cdot|_{f}$ is trivial on units, thus on $U(3 D)$, while $\chi_{D}$ is invariant on $U(3 D)$ by definition. Moreover, $\varphi$ is trivial on all the units at all the unramified places, while, at $3, \varphi$ is invariant under $1+3 \mathbb{Z}_{3}[\omega]$, thus it is trivial on all of $U(3 D)$. Thus we can take the quotient by $U(3 D)$ as well. Note that the integral is now a finite sum:

$$
\begin{aligned}
& Z_{f}\left(s, \chi_{D} \varphi, \Phi_{f}\right) \\
& =\operatorname{vol}(U(3 D)) \sum_{\alpha_{f}^{\prime \prime} \in U(3 D) \backslash \mathbb{A}_{K, f}^{\times} / K^{\times}}\left(\sum_{k \in K^{\times}} \frac{k}{|k|_{\mathbb{C}}^{2 s}} \Phi_{f}\left(k \alpha_{f}^{\prime \prime}\right)\right) \chi_{D, f}\left(\alpha_{f}^{\prime \prime}\right) \varphi_{f}\left(\alpha_{f}^{\prime \prime}\right)\left|\alpha_{f}^{\prime \prime}\right|_{f}^{s} .
\end{aligned}
$$

We compute $\operatorname{vol}(U(3 D))=\operatorname{vol}\left(1+3 \mathbb{Z}_{3}[\omega]\right) \prod_{p \mid D} \operatorname{vol}\left(\mathbb{Z}+D \mathscr{O}_{K_{p}}\right)=V_{3 D}$ and, changing notation, we get:

$$
Z_{f}\left(s, \chi_{D, f} \varphi_{f}, \Phi_{f}\right)=V_{3 D} \sum_{\alpha_{f} \in U(3 D) \backslash \mathbb{A}_{K, f}^{\times} / K^{\times}} I\left(s, \alpha_{f}, \Phi_{f}\right) \chi_{D, f}(\alpha) \varphi_{f}(\alpha) .
$$

Finally together with (6) we get the result of the lemma.

\subsection{Representative classes of $\mathrm{Cl}\left(\mathscr{O}_{3 D}\right)$}

We will use the following lemma (see [22]) that is easy to show:

Lemma 7 Any primitive ideal of $\mathscr{O}_{K}$ can be written in the form $\mathscr{A}=\left[a, \frac{-b+\sqrt{-3}}{2}\right]_{\mathbb{Z}}$ as $a \mathbb{Z}$-module, where $b$ is an integer (determined only modulo $2 a$ ) such that $b^{2} \equiv-3$ $\bmod 4 a$ and $\mathrm{Nm} \mathscr{A}=a$.

Conversely, given an integer satisfying the above congruence and $\mathscr{A}$ defined as above, we get that $\mathscr{A}$ is an ideal in $\mathscr{O}_{K}$ of norm a.

We will use the notation $k_{\mathscr{A}}$ for the generator $k_{\mathscr{A}} \equiv 1 \bmod 3$ of a primitive ideal $\mathscr{A}$ in $\mathscr{O}_{K}$. If we choose a lattice such that $\mathscr{A}=\left[a, \frac{-b+\sqrt{-3}}{2}\right]_{\mathbb{Z}}$, we denote the corresponding CM point $\tau_{\mathscr{A}}=\frac{-b+\sqrt{-3}}{2 a}$.

We can write adelically $\mathrm{Cl}\left(\mathscr{O}_{3 D}\right) \simeq U(3 D) \backslash \mathbb{A}_{K, f}^{\times} / K^{\times}$. This follows from the Strong approximation theorem, as $K$ is a PID and thus we have $U(3 D) \backslash \mathbb{A}_{K, f}^{\times} / K^{\times} \cong$ $\left(\prod_{p \mid 3 D}\left(\mathscr{O}_{K_{p}}\right)^{\times} /\left(\mathbb{Z}+3 D \mathscr{O}_{K_{p}}\right)^{\times}\right) /\langle-\omega\rangle$. Then we can define the map

$$
\prod_{p \mid 3 D}\left(\mathscr{O}_{K_{p}}\right)^{\times} /\left(\mathbb{Z}+3 D \mathscr{O}_{K_{p}}\right)^{\times} /\langle-\omega\rangle \rightarrow I(3 D) / P_{\mathbb{Z}, 3 D}
$$


given by

$$
\left(\alpha_{v}\right)_{v \mid 3 D} \rightarrow\left(k_{\alpha}\right)
$$

where we choose the representative $\pm \alpha \omega^{k}$, such that $\alpha_{3} \equiv 1 \bmod 3$, and $k_{\alpha}$ is an element of $\mathscr{O}_{K}$ such that $k_{\alpha} \equiv \alpha_{v} \bmod 3 D$. Note that this is well defined as $\left(k_{\alpha}\right)$ gives us a unique class in $\mathrm{Cl}\left(\mathscr{O}_{3 D}\right)$, and two elements $\alpha_{1}, \alpha_{2}$ get sent to the same class in $\mathrm{Cl}\left(\mathscr{O}_{3 D}\right)$ only if $\alpha_{1} \equiv \alpha_{2} \bmod 3 D$.

Thus for $\alpha_{f} \in \widehat{\mathscr{O}}_{K}$ we can choose a class $\left[\mathscr{A}_{\alpha}\right]$ in $\mathrm{Cl}\left(\mathscr{O}_{3 D}\right)$ by taking a representative $\mathscr{A}_{\alpha_{f}}=\left(k_{\alpha}\right)$, for $k_{\alpha} \in \mathscr{O}_{K}$ such that $k_{\alpha} \equiv \alpha_{p} \bmod 3 D \mathscr{O}_{K_{p}}$ for $p \mid 3 D$. Note that this choice is not unique. However, we can pick the representatives $\mathscr{A}_{\alpha}$ to be primitive ideals.

Thus we can further write $\mathscr{A}_{\alpha}$ as a $\mathbb{Z}$-lattice $\mathscr{A}_{\alpha}=\left[a, \frac{-b+\sqrt{-3}}{2}\right]_{\mathbb{Z}}$, where $a=$ $\mathrm{Nm} \mathscr{A}_{\alpha}$ and $b$ is chosen (not uniquely) such that $b^{2} \equiv-3 \bmod 4 a$. We define the corresponding CM point $\tau_{\mathscr{A}_{\alpha}}=\frac{-b+\sqrt{-3}}{2 a}$.

\subsection{Eisenstein series of weight 1}

We will now connect $I\left(s, \alpha_{f}, \Phi_{f}\right)=\sum_{k \in K^{\times}} \frac{k}{|k|_{\mathbb{C}}^{2 s}} \Phi_{f}\left(k \alpha_{f}\right)$ to an Eisenstein series. We define the following classical Eisenstein series of weight 1 :

$$
E_{\varepsilon}(s, z)=\sum_{m, n}^{\prime} \frac{\varepsilon(n)}{(3 m z+n)|3 m z+n|^{s}} .
$$

Here the sum is taken over all $m, n \in \mathbb{Z}$ except for the pair $(0,0)$, and $\varepsilon=(\dot{\overline{3}})$ is the quadratic character associated to the field extension $K / \mathbb{Q}$. Note also that from quadratic reciprocity we have $\left(\frac{-3}{\cdot}\right)=\left(\frac{\dot{3}}{3}\right)$.

The Eisenstein series $E_{\varepsilon}(s, z)$ does not converge absolutely for $s=0$, but we can still compute its value using the Hecke trick (see [11]). We compute its Fourier expansion at $s=0$ in the following section.

Using this notation, we have the following equality:

Lemma 8 For $\alpha_{f} \in \prod_{v \nmid \infty} \mathscr{O}_{K_{v}}^{\times}$, let $\mathscr{A}_{\alpha_{f}}=\left(k_{\alpha}\right)$ be a choice of an ideal in the corresponding class of $\mathrm{Cl}\left(\mathscr{O}_{3 D}\right)$. We write $\mathscr{A}_{\alpha}=\left[a_{\alpha}, \frac{-b+\sqrt{-3}}{2}\right]_{\mathbb{Z}}$ and take $\tau_{\mathscr{A}_{\alpha}}=\frac{-b+\sqrt{-3}}{2 a_{\alpha}}$ the corresponding CM point. Then we have:

$$
I\left(s, \alpha_{f}, \Phi_{f}\right)=\frac{1}{2} \frac{\left(\mathrm{Nm} \mathscr{A}_{\alpha}\right)^{1-s}}{k_{\alpha}} E_{\varepsilon}\left(s, D \tau \overline{\mathscr{A}_{\alpha}}\right) .
$$

Remark 9 Note that the variable $\tau_{\overline{\mathscr{A}}_{\alpha}}$ on the left hand side is not uniquely defined. However, the right-hand side depends only on the class $\left[\mathscr{A}_{\alpha}\right]$ in $\mathrm{Cl}\left(\mathscr{O}_{3 D}\right)$.

Proof Recall that $I\left(s, \alpha_{f}, \Phi_{f}\right)=\sum_{k \in K^{\times}} \frac{k}{|k|_{\mathbb{C}}^{2 s}} \Phi_{f}\left(k \alpha_{f}\right)$. We need to compute $\Phi_{f}\left(k \alpha_{f}\right)$. Note that $\Phi_{v}\left(k \alpha_{v}\right) \neq 0$ only for $k \alpha_{v} \in \mathscr{O}_{K_{v}}$ at all places $v$, and since 
$\alpha_{v} \in \mathscr{O}_{K_{v}}^{\times}$, we must have $k \in \mathscr{O}_{K_{v}}$ as well for all $v$. This implies $k \in \mathscr{O}_{K}$ and for all places $v \nmid 3 D$ we get $\Phi_{v}\left(k \alpha_{v}\right)=1$ for $k \in \mathscr{O}_{K}$. Thus we can rewrite:

$$
I\left(s, \alpha_{f}, \Phi_{f}\right)=\sum_{k \in \mathscr{O}_{K}} \frac{k}{|k|_{\mathbb{C}}^{2 s}} \Phi_{3 D}\left(k \alpha_{3 D}\right)
$$

where $\Phi_{3 D}=\prod_{v \mid 3 D} \Phi_{v}$ and $\alpha_{3 D}=\left(\alpha_{v}\right)_{v \mid 3 D}$.

We can further compute $\Phi_{v}\left(k \alpha_{v}\right)$ for $v \mid 3 D$. Recall that we defined the SchwartzBruhat functions $\Phi_{p}=\operatorname{char}_{\left(\mathbb{Z}+3 D \mathscr{O}_{K p}\right)} \times$ for $p \mid D$ and $\Phi_{3}=\operatorname{char}_{\left(1+3 \mathbb{Z}_{3}[\omega]\right)}$. Then we have $\Phi_{3 D}\left(k \alpha_{3 D}\right) \neq 0$ iff $k \alpha_{p} \in a+3 D \mathscr{O}_{K_{p}}$ for some integer $a,(a, p)=1$ and, for $p=3, k \alpha_{3} \in 1+3 \mathscr{O}_{K_{3}}$.

Recall that we defined $k_{\alpha}$ such that $k_{\alpha} \equiv \alpha_{p} \bmod 3 D \mathscr{O}_{K_{p}}$ for all $p \mid 3 D$. Then $k k_{\alpha} \in a+3 D \mathscr{O}_{K_{p}}$ for $(a, p)=1$ and $k k_{\alpha} \in 1+3 \mathbb{Z}_{3}[\omega]$. Furthermore, for $k \in \mathscr{O}_{K}$ we actually have $\Phi_{3 D}\left(k \alpha_{3 D}\right)=\Phi_{3 D}\left(k k_{\alpha}\right)$. Then we can rewrite $I\left(s, \alpha_{f}, \Phi_{f}\right)$ using $k_{\alpha}$ as $I\left(s, \alpha_{f}, \Phi_{f}\right)=\sum_{k \in \mathscr{O}_{K}} \frac{k}{|k|_{\mathbb{C}}^{2 s}} \Phi_{3 D}\left(k k_{\alpha}\right)$. We can rewrite this further:

$$
I\left(s, \alpha_{f}, \Phi_{f}\right)=\frac{\left|k_{\alpha}\right|_{\mathbb{C}}^{2 s}}{k_{\alpha}} \sum_{k \in \mathscr{O}_{K}} \frac{k k_{\alpha}}{\left|k k_{\alpha}\right|_{\mathbb{C}}^{2 s}} \Phi_{3 D}\left(k k_{\alpha}\right),
$$

Finally, we will make this explicit. Note that we must have $k k_{\alpha} \in \mathscr{A}_{\alpha}$, where $\mathscr{A}_{\alpha}=$ $\left(k_{\alpha}\right)$, as well as $k k_{\alpha} \in a_{p}+D \mathscr{O}_{K_{p}}$ for some integer $a_{p},\left(a_{p}, p\right)=1$, and $k k_{\alpha} \in 1+$ $3 \mathbb{Z}_{3}[\omega]$. By the Chinese remainder theorem, we can find an integer $a$ such that $a \equiv a_{p}$ $\bmod D$ and $a \equiv 1 \bmod 3$. Then $k k_{\alpha} \in\left(a+D \prod_{p \mid 3 D} \mathscr{O}_{K_{p}}\right) \cap \mathscr{O}_{K}$, thus $k k_{\alpha} \in P_{\mathbb{Z}, 3 D} \cap$ $P_{1,3}$. Here $P_{\mathbb{Z}, 3 D}=\left\{k \in K: k \equiv a \bmod 3 D \mathscr{O}_{K}\right.$ for some integer $\left.a,(a, 3 D)=1\right\}$ and $P_{1,3}=\{k \in K: k \equiv 1 \bmod 3\}$. We rewrite:

$$
I\left(s, \alpha_{f}, \Phi_{f}\right)=\frac{\left|k_{\alpha}\right|_{\mathbb{C}}^{2 s}}{k_{\alpha}} \sum_{k \in \mathscr{A}_{\alpha} \cap P_{\mathbb{Z}, D} \cap P_{1,3}} \frac{k}{|k|_{\mathbb{C}}^{2 s}}
$$

Finally, we want to write the elements of $\mathscr{A}_{\alpha} \cap P_{\mathbb{Z}, D} \cap P_{1,3}$ explicitly. Recall that we can write $\mathscr{A}_{\alpha}$ as a $\mathbb{Z}$-lattice $\mathscr{A}_{\alpha}=\left[a, \frac{-b+\sqrt{-3}}{2}\right]_{\mathbb{Z}}$. Then all of the elements of $\mathscr{A}$ are of the form $m a+n \frac{-b+\sqrt{-3}}{2}$ for some integers $m, n \in \mathbb{Z}$. Moreover, note that the intersection of $\mathscr{A}$ and $P_{\mathbb{Z}, 3 D}=\left\{k \in \mathscr{O}_{K}: k \equiv n \bmod 3 D\right.$, for some integer $\left.n,(n, 3 D)=1\right\}$ is $\left\{m a+3 D n \frac{-b+\sqrt{-3}}{2}: m, n \in \mathbb{Z}\right\}$. Further taking the intersection with $P_{1,3}$, we must have $m a \equiv 1$, thus, as $a$ is norm in $\mathscr{O}_{K}, m \equiv 1 \bmod 3$, and we can rewrite $I\left(s, \alpha_{f}, \Phi_{f}\right)$ in the form:

$$
I\left(s, \alpha_{f}, \Phi_{f}\right)=\frac{a^{s}}{k_{\alpha}} \sum_{m, n \in \mathbb{Z}, m \equiv 1(\bmod 3)} \frac{m a+n \frac{-b+\sqrt{-3}}{2}}{\left|m a+3 n D \frac{-b+\sqrt{-3}}{2}\right|_{\mathbb{C}}^{2 s}} .
$$


By changing $n \rightarrow-n$ and taking out a factor of $a^{1-2 s}$, we have:

$$
I\left(s, \alpha_{f}, \Phi_{f}\right)=\frac{a^{1-s}}{k_{\alpha}} \sum_{\substack{m, n \in \mathbb{Z}, m \equiv 1(\bmod 3)}} \frac{1}{\left(m+n \frac{b+\sqrt{-3}}{2 a}\right)\left|m+3 n D \frac{b+\sqrt{-3}}{2 a}\right|_{\mathbb{C}}^{2 s-2}} .
$$

Note that for $\operatorname{Re}(s)>1$ the integral converges absolutely, and we can rewrite the sum as:

$$
I\left(s, \alpha_{f}, \Phi_{f}\right)=\frac{1}{2} \frac{a^{1-s}}{k_{\alpha}} \sum_{m, n \in \mathbb{Z}} \frac{\varepsilon(m)}{\left(m+3 n D \frac{b+\sqrt{-3}}{2 a}\right)\left|m+3 n D \frac{b+\sqrt{-3}}{2 a}\right|_{\mathbb{C}}^{2 s-2}},
$$

where $\varepsilon(m)=\left(\frac{m}{3}\right)$ is the usual quadratic character. On the right hand side we recognize the Eisenstein series $E_{\varepsilon}\left(2 s-2, \tau \overline{\mathscr{A}_{\alpha}}\right)$ and we get $I\left(s, \alpha_{f}, \Phi_{f}\right)=\frac{1}{2} \frac{a^{1-s}}{k_{\alpha}} E_{\varepsilon}(2 s-$ $2, D \tau_{\mathscr{A}_{\alpha}}$ ). By analytic continuation, we can extend the equality to all $s \in \mathbb{C}$.

Now we can rewrite the linear combination in Lemma 6 by taking representatives $\mathscr{A}$ for the classes of $\operatorname{Cl}\left(\mathscr{O}_{3 D}\right)$. Note that for $\alpha \in \widehat{\mathscr{O}}_{K}^{\times}$with $\alpha \equiv 1 \bmod 3$ we have $\varphi_{f}\left(\alpha_{f}\right)=1$ and

$$
\chi_{D}\left(\alpha_{f}\right)=\chi_{D}\left((\alpha)_{p \mid 3 D}\right)=\chi_{D}\left(\left(k_{\alpha}\right)_{p \mid 3 D}\right)=\chi_{D}^{-1}\left(\left(k_{\alpha}\right)_{p \nmid 3 D}\right)=\overline{\chi_{D}\left(\mathscr{A}_{\alpha}\right)} .
$$

Using the lemma above and after inverting each class $\mathscr{A} \rightarrow \overline{\mathscr{A}}$ in $\mathrm{Cl}\left(\mathscr{O}_{3 D}\right)$, we get:

Corollary 10 For all s, taking representative ideals $\mathscr{A}=\left[a, \frac{-b+\sqrt{-3}}{2}\right]_{\mathbb{Z}}$ for the classes in the ring class group $\mathrm{Cl}\left(\mathscr{O}_{3 D}\right)$, we have:

$$
L\left(s, \chi_{D} \varphi\right)=\frac{1}{2} \sum_{[\mathscr{A}] \in \operatorname{Cl}\left(\mathscr{O}_{3 D}\right)} E_{\varepsilon}\left(2 s-2, D \tau_{\mathscr{A}}\right) \chi_{D}(\mathscr{A}) \frac{(\mathrm{Nm} \mathscr{A})^{1-s}}{k_{\mathscr{A}}},
$$

where $\mathscr{A}=\left(k_{\mathscr{A}}\right)$ with $k_{\mathscr{A}} \equiv 1 \bmod 3$ and $\tau_{\mathscr{A}}=\frac{-b+\sqrt{-3}}{2 a}$ the associated CMpoints.

\subsection{Fourier expansion of $E_{\varepsilon}(s, z)$ at $s=0$}

We want to connect the Eisenstein series $E_{\varepsilon}(s, z)=\sum_{c, d}{ }^{\prime} \frac{\varepsilon(d)}{(3 c z+d)|3 c z+d|^{2 s}}$, where $\sum_{c, d}{ }^{\prime}$ denotes the sum over all $c, d \in \mathbb{Z}$ with $(c, d) \neq(0,0)$, to the theta function

$$
\Theta_{K}(z)=\sum_{m, n \in \mathbb{Z}} e^{2 \pi i\left(m^{2}+n^{2}-m n\right) z}
$$

associated to the number field $K$. It is a modular form of weight 1 for the congruence group $\Gamma_{1}(3)$. Note that this differs from the theta function $\Theta_{K}$ chosen by RodriguezVillegas and Zagier in [24] by a factor of $1 / 2$. 
More precisely, we are going to show the following version of the Siegel-Weil theorem:

Theorem $11 E_{\varepsilon}(0, z)=2 L(1, \varepsilon) \Theta_{K}(z)$.

Proof We will show this by computing the Fourier expansion of $E_{\varepsilon}(s, z)$ at $s=0$ using the Hecke trick and comparing it to the Fourier expansion of $\Theta_{K}(z)$. We will follow closely the exposition of Pacetti [20]. This is also done by Hecke in [11]. We first rewrite $E_{\varepsilon}(s, z)$ in the form:

$$
E_{\varepsilon}(z, s)=\sum_{d} \frac{\varepsilon(d)}{d^{1+2 s}}+2 \sum_{c=1}^{\infty} \sum_{r=0}^{2} \frac{\varepsilon(r)}{3^{2 s+1}} \sum_{d \in \mathbb{Z}} \frac{\varepsilon(r)}{\left(\frac{3 c z+r}{3}+d\right)\left|\frac{3 c z+r}{3}+d\right|^{2 s}}
$$

where $\sum_{d}{ }^{\prime}$ denotes the sum over all $d \in \mathbb{Z}, d \neq 0$. We define for $z$ in the upper-half plane $H(z, s)=\sum_{m \in \mathbb{Z}} \frac{1}{(z+m)|z+m|^{2 s}}$ and then we can rewrite the form above as:

$$
E_{\varepsilon}(s, z)=2 L(1+2 s, \varepsilon)+2 \sum_{c=1}^{\infty} \sum_{r=0}^{2} \frac{\varepsilon(r)}{3^{2 s+1}} H\left(\frac{3 d z+r}{3}, s\right) .
$$

Pacetti [20], following Shimura [29, Lemma 1, p. 84], computed the Fourier expansion of $H(z, s)$ when $s \rightarrow 0$ to be $\lim _{s \rightarrow 0} H(s, z)=-\pi i-2 \pi i \sum_{n=1}^{\infty} q^{n}$. It gives us in the relation above:

$$
E_{\varepsilon}(0, z)=2 L(1, \varepsilon)+2 \sum_{c=1}^{\infty} \sum_{r=0}^{2} \frac{\varepsilon(r)}{3}\left(-\pi i-2 \pi i \sum_{n=1}^{\infty} e^{2 \pi i n z c} \omega^{n r}\right) .
$$

We compute separately the inner sum and get:

$$
\sum_{r=0}^{2} \frac{\varepsilon(r)}{3}\left(-\pi i+\sum_{n=1}^{\infty} e^{2 \pi i n z c} \omega^{n r}\right)=-\frac{2 \pi i}{3} G(\varepsilon) \sum_{n=1}^{\infty} e^{2 \pi i n z c} \varepsilon(n),
$$

where $G(\varepsilon)=\sum_{r=0}^{2} \varepsilon(r) \omega^{r}=\sqrt{-3}$ is the quadratic Gauss sum corresponding to $\varepsilon$. Then we can rewrite:

$$
E_{\varepsilon}(0, z)=2 L(1, \varepsilon)+\frac{4 \pi \sqrt{3}}{3} \sum_{N=1}^{\infty}\left(\sum_{m \mid N} \varepsilon(m)\right) e^{2 \pi i N z}
$$

Since $\varepsilon$ is a quadratic character, we compute $L(1, \varepsilon)=\frac{\pi \sqrt{3}}{9}$ (see Kowalski [18]) and this gives us the Fourier expansion:

$$
E_{\varepsilon}(0, z)=\frac{2 \pi \sqrt{3}}{9}\left(1+6 \sum_{N=1}^{\infty}\left(\sum_{m \mid N} \varepsilon(m)\right) e^{2 \pi i N z}\right) .
$$


It is easy to show that $\sum_{m \mid n} \varepsilon(m)$ represents the number of ideals of norm $n$ in $\mathscr{O}_{K}$, thus we got $\frac{2 \pi \sqrt{3}}{9}\left(1+6 \sum_{\mathscr{A}} e^{2 \pi i(\mathrm{Nm} \mathscr{A}) z}\right)$. Moreover $\left|\mathscr{O}_{K}^{\times}\right|=6$ and $\mathrm{Nm}_{K / \mathbb{Q}}(m+$ $n \omega)=m^{2}+n^{2}-m n$ and thus we can recognize the sum in the bracket on the RHS of (7) to equal the theta function $\Theta_{K}(z)$, which finishes the proof.

\subsection{Formula for $L\left(1, \chi_{D} \varphi\right)$}

Applying Corollary 10 for $s=1$ we get

$$
L\left(1, \chi_{D} \varphi\right)=\frac{1}{2} \sum_{[\mathscr{A}] \in \operatorname{Cl}\left(\mathscr{O}_{3 D}\right)} \frac{1}{\bar{k}_{\mathscr{A}}} E_{\varepsilon}\left(0, D \tau_{\mathscr{A}}\right) \chi_{D}(\mathscr{A}) .
$$

Furthermore, from Theorem 11, this is the same as:

$$
L\left(1, \chi_{D} \varphi\right)=\frac{\pi \sqrt{3}}{9} \sum_{[\mathscr{A}] \in \operatorname{Cl}\left(\mathscr{O}_{3 D}\right)} \frac{1}{\bar{k}_{\mathscr{A}}} \Theta_{K}\left(D \tau_{\mathscr{A}}\right) \chi_{D}(\mathscr{A}) .
$$

We need one more step before rewriting the formula as a trace. We will use the following lemma:

Lemma 12 For $\mathscr{A}=\left[a, \frac{-b+\sqrt{-3}}{2}\right]_{\mathbb{Z}}$ a primitive ideal of norm $\mathrm{Nm} \mathscr{A}=a$, with generator $\mathscr{A}=\left(k_{\mathscr{A}}\right)$, where $k_{\mathscr{A}} \equiv 1 \bmod 3$ and $\tau_{\mathscr{A}}=\frac{-b+\sqrt{-3}}{2}$, we have:

$$
\Theta_{K}\left(\tau_{\mathscr{A}}\right)=\overline{k_{\mathscr{A}}} \Theta_{K}(\omega)
$$

Proof Since $\mathscr{A}=\left[a, \frac{-b+\sqrt{-3}}{2}\right]_{\mathbb{Z}}$ as a $\mathbb{Z}$-lattice, we can write its generator $k_{\mathscr{A}}$ in the form $k_{\mathscr{A}}=m a+3 n \frac{-b+\sqrt{-3}}{2}$ for some integers $m, n$ such that $m \equiv 1(3)$ and $\operatorname{gcd}(m, 3 n)=1$. Then we can find integers $A, B$ such that $m A+3 n B=1$, and thus $\left(\begin{array}{cc}A & B \\ 3 n & m\end{array}\right)$ is a matrix in $\Gamma_{1}(3)$. Since $\Theta(z)$ is a modular form of weight 1 for $\Gamma_{1}(3)$, we have $\Theta_{K}\left(\left(\begin{array}{cc}A & B \\ 3 n & m\end{array}\right) \tau_{\mathscr{A}}\right)=\left(m+3 n \tau_{\mathscr{A}}\right) \Theta_{K}\left(\tau_{\mathscr{A}}\right)$.

Noting that $3 n \tau_{\mathscr{A}}+m=k_{\mathscr{A}} / a=1 / \overline{k_{\mathscr{A}}}$, we can compute the term on the LHS to be $\Theta_{K}\left(\left(A \tau_{\mathscr{A}}+B\right) \overline{k_{\mathscr{A}}}\right)$ and, after expanding, we are evaluating $\Theta_{K}$ at $-3 n A \frac{b^{2}+3}{4 a}+$ $a b B+\frac{b(-m A+3 n B)}{2}+\frac{\sqrt{-3}}{2}$. Note that $m A-3 n B=1$ implies that $m A$ and $3 n B$ have different parities. Also, $b$ is odd and $b^{2}+3 \equiv 0 \bmod 4 a$. Then $-3 n A \frac{b^{2}+3}{4 a}+a b B+$ $\frac{b(-m A+3 n B)+1}{2} \in \mathbb{Z}$ and thus using the period 1 of $\Theta_{K}$ we get $\Theta_{K}\left(\left(\begin{array}{cc}A & B \\ 3 n & m\end{array}\right) \tau_{\mathscr{A}}\right)=$ $\Theta_{K}(\omega)$. This finishes the proof.

Using the Lemma above we can rewrite (8) as:

$$
L\left(1, \varphi \chi_{D}\right)=\frac{\pi \sqrt{3}}{9} \Theta_{K}(\omega) \sum_{[\mathscr{A}] \in \operatorname{Cl}\left(\mathscr{O}_{3 D}\right)} \frac{\Theta_{K}\left(D \tau_{\mathscr{A}}\right)}{\Theta_{K}\left(\tau_{\mathscr{A}}\right)} \chi_{D}(\mathscr{A})
$$


Now we will rewrite the formula (9) as a trace. We can define $f(z)=\frac{\Theta_{K}(D z)}{\Theta_{K}(z)}$ and this is a modular function for $\Gamma_{0}(3 D)$. We will prove in Sect. 5 in Proposition 39 that $f(\omega) \in H_{3 D}$, the ring class field of corresponding to the order $\mathscr{O}_{3 D}=\mathbb{Z}+3 D \mathscr{O}_{K}$. Moreover, we show in the same proposition that, for $\mathscr{A}=\left[a, \frac{-b+\sqrt{-3}}{2}\right]_{\mathbb{Z}}$ a primitive ideal in $\mathscr{O}_{K}$, we have the Galois conjugate:

$$
f(\omega)^{\sigma_{\mathscr{A}}^{-1}}=f\left(\tau_{\mathscr{A}}\right)
$$

where $\sigma_{\mathscr{A}}$ is the Galois action corresponding to the ideal $\mathscr{A}$ via the Artin map.

Furthermore, from we have $\left(D^{1 / 3}\right)^{\sigma_{\mathscr{A}}^{-1}}=D^{1 / 3} \chi_{D}(\mathscr{A})$ and then formula (9) becomes:

$$
L\left(E_{D}, 1\right)=\frac{\pi \sqrt{3}}{9} D^{-1 / 3} \Theta_{K}(\omega) \sum_{[\mathscr{A}] \in \operatorname{Cl}\left(\mathscr{O}_{3 D}\right)}\left(D^{1 / 3} \frac{\Theta_{K}(D \omega)}{\Theta_{K}(\omega)}\right)^{\sigma_{\mathscr{A}}-1}
$$

Moreover, $D^{1 / 3} \in H_{3 D}$ (see for example Cohn [4]). Thus we can rewrite the sum on the left hand side as $\operatorname{Tr}_{H_{3 D} / K}\left(D^{1 / 3} \frac{\Theta_{K}(D \omega)}{\Theta_{K}(\omega)}\right)$. We can compute the extra terms as well. Rodriguez-Villegas and Zagier in [24] cite $\Theta_{K}\left(\frac{-9+\sqrt{-3}}{18}\right)=-6 \Gamma\left(\frac{1}{3}\right)^{3} /(2 \pi)^{2}$. Using several of the properties of $\Theta_{K}$ proved in the Appendix ((27) and Lemma 41), we can compute $\Theta(\omega)=\Gamma\left(\frac{1}{3}\right)^{3} /\left(2 \pi^{2}\right)$.

As the real period $\Omega_{D}$ of the elliptic curve $E_{D}$ is $\Omega_{D}=D^{-1 / 3} \frac{\sqrt{3} \Gamma\left(\frac{1}{3}\right)^{3}}{6 \pi}$, we get the formula of Theorem 5:

$$
L\left(E_{D}, 1\right)=\frac{\Omega_{D}}{3} \operatorname{Tr}_{H_{3 D} / K}\left(D^{1 / 3} \frac{\Theta_{K}(D \omega)}{\Theta_{K}(\omega)}\right) .
$$

Note that this implies $S_{D} \in K$. Moreover, as $D^{1 / 3} \Theta(D \omega) / \Theta(\omega)$ is invariant under complex conjugation, we get $S_{D} \in \mathbb{R}$. Since $K \cap \mathbb{R}=\mathbb{Q}$, this furthermore implies $S_{D} \in \mathbb{Q}$. We will show in Sect. 4.5 that actually $3 c_{3 D} S_{D} \in \mathbb{Z}$.

Remark 13 If we take $D=D_{1} D_{2}^{2}$ such that $D_{0}=D_{1} D_{2}$ is square-free, note that the character $\chi_{D}=\chi_{D_{1}} \overline{\chi_{D_{2}}}$ is well defined on the class group $\mathrm{Cl}\left(\mathscr{O}_{3 D_{0}}\right)$. Then the above computations work for $D_{0}$ and the character $\chi_{D}=\chi_{D_{1}} \overline{\chi_{D_{2}}}$ instead of $\chi_{D_{0}}=\chi_{D_{1}} \chi_{D_{2}}$ and we get:

$$
L\left(1, \chi_{D} \varphi\right)=\frac{\pi \sqrt{3}}{9} \Theta_{K}(\omega) \sum_{[\mathscr{A}] \in \operatorname{Cl}\left(\mathscr{O}_{3 D_{0}}\right)} \frac{\Theta_{K}\left(D_{0} \tau_{\mathscr{A}}\right)}{\Theta_{K}\left(\tau_{\mathscr{A}}\right)} \chi_{D}(\mathscr{A}) .
$$

Note that, for $D=D_{1} D_{2}^{2}, L\left(E_{D}, s\right)=L\left(s, \chi_{D_{1}} \overline{\chi_{D_{2}}} \varphi\right)$ and thus we have:

$$
S_{D}=\frac{1}{3} \sum_{[\mathscr{A}] \in \operatorname{Cl}\left(\mathscr{O}_{3 D_{0}}\right)} \frac{\Theta_{K}\left(D_{0} \tau_{\mathscr{A}}\right)}{\Theta_{K}\left(\tau_{\mathscr{A}}\right)} \chi_{D}(\mathscr{A}) D^{1 / 3}
$$


As before we have $\left(D_{1}^{1 / 3} D_{2}^{2 / 3}\right)^{\sigma_{\mathscr{A}}^{-1}}=D_{1}^{1 / 3} \chi_{D_{1}}(\mathscr{A}) D_{2}^{2 / 3} \overline{\chi_{D_{2}}(\mathscr{A})}$ and finally we can write the expression above as:

$$
L\left(E_{D}, 1\right)=\frac{\Omega_{D}}{3} \operatorname{Tr}_{H_{3 D_{0}} / K}\left(D^{1 / 3} \frac{\Theta_{K}\left(D_{0} \omega\right)}{\Theta_{K}(\omega)}\right) .
$$

\section{Second formula for $S_{D}$}

For $r \in \mathbb{Z}, \mu \in\{1 / 2,1 / 6\}$, we define the theta functions of weight $1 / 2$ :

$$
\theta_{r, \mu}(z)=\sum_{n \in \mathbb{Z}} e^{\pi i(n+r / D-\mu)^{2} z}(-1)^{n} .
$$

Throughout the paper we will use the notation $r \in \mathbb{Z} / D \mathbb{Z}$ to mean any family of representatives for the residues $r \bmod D$. We denote $\theta_{0}=\theta_{0,1 / 6}$. Note that $\theta_{0}(z)=$ $\eta(z / 3)$, where $\eta$ is the Dedekind eta function, while $\sum_{\substack{r \in \mathbb{Z} / D \mathbb{Z} \\ r \equiv 1(6)}} \theta_{r, 1 / 6}(z)=\eta\left(\frac{z}{3 D^{2}}\right)$.

In this section we will use a Factorization formula of Rodriguez-Villegas and Zagier from [23] to show the following theorem, that is a restatement of Theorem 2 from the Introduction:

Theorem 14 In the case of $D=\prod_{p_{i} \equiv 1(3)} p_{i}^{e_{i}}$, let $D_{0}=\prod_{p_{i} \mid D} p_{i}$ be the radical of $D$ and $\sigma(D)$ the number of distinct prime divisors of $D$. Then $S_{D}$ is an integer square up to an even power of 3 and we have:

$$
S_{D}=\frac{(-1)^{\sigma(D)}}{3^{\sigma(D)+2}} T_{D}^{2}
$$

where $T_{D} / 3 \in \mathbb{Z}$ if $\sigma(D)$ is even and $T_{D} / \sqrt{-3} \in \mathbb{Z}$ if $\sigma(D)$ is odd. We have the exact formula:

$$
T_{D}=\frac{\omega^{k_{0}}}{\varphi\left(D_{0}\right)} \operatorname{Tr}_{H_{\mathscr{O}} / K}\left(\frac{\theta_{1,1 / 6}(\tau)}{\theta_{0}(\tau)} \bar{\pi}_{1}^{-2 / 3} \pi_{2}^{1 / 3}\right) .
$$

Here $\tau=\frac{-b+\sqrt{-3}}{2}$ is a CM-point, with $b^{2} \equiv-3 \bmod 12 D^{2}, \pi_{1}, \pi_{2}$ are elements in $\mathscr{O}_{K}$ such that $\pi_{1}, \pi_{2} \equiv 1 \bmod 3, \pi_{1} \pi_{2}$ has norm $D_{0}$ and $\pi_{1} \pi_{2}^{2}$ has norm $D$, and such that $\left(\pi_{1} \pi_{2}\right)^{2}$ divides the ideal $\left(\frac{-b+\sqrt{-3}}{2}\right), H_{\mathscr{O}}$ is the ray class field of modulus $3 D_{0}$ and $\omega^{k_{0}}$ is the unique cube root of unity that makes $T_{D}$ real or purely imaginary.

Below we discuss the details of $D$ square-free. All definitions and proofs can be easily extended to all $D=D_{1} D_{2}^{2}$ by using the formula (13) with $D_{0}=D_{1} D_{2}$ squarefree. We do this in Sect. 4.6.

Take $\tau=\frac{-b+\sqrt{-3}}{2}$ a CM point such that $b^{2} \equiv-3 \bmod 12 D^{2}$ and an element $\pi \equiv 1 \bmod 3$ of norm $D$ in $\mathscr{O}_{K}$ such that $\pi^{2}$ divides the ideal $\left(\frac{-b+\sqrt{-3}}{2}\right)$.

We will use the notation: 
$-\Theta_{\mu}(\tau)= \begin{cases}\frac{3}{2} \Theta_{K}(\tau)-\frac{1}{2} \Theta_{K}(\tau / 3), & \text { for } \mu=1 / 6 \\ \Theta_{K}(\tau / 3), & \text { for } \mu=1 / 2 .\end{cases}$

$-S_{\mu}=\frac{2}{3} \sum_{[\mathscr{A}] \in \operatorname{Cl}\left(\mathscr{O}_{3 D}\right)} \frac{\Theta_{\mu}\left(D \tau_{\mathscr{A}}\right)}{\Theta_{K}\left(\tau_{\mathscr{A}}\right)} \chi_{D}(\mathscr{A}) D^{1 / 3}$.

In Theorem 3 we have proved that $3 c_{3 D} S_{D}=S_{1 / 6}+1 / 2 S_{1 / 2}$. We are actually going to show in Corollary 23 that $S_{1 / 2}=0$, thus it is enough to compute the formula (14) for $S_{1 / 6}$.

Using a Factorization formula of Rodriguez-Villegas and Zagier from [23] we will write the theta functions $\Theta_{\mu}$ of weight 1 as linear combinations of products of theta functions of weight $1 / 2$ in Proposition 17. We define:

$$
R_{D, \mu}(z)=\sum_{\substack{r \in(\mathbb{Z} / D \mathbb{Z})^{\times} \\ r \equiv 1(6)}} \frac{\theta_{r, \mu}(3 z)}{\theta_{0}(3 z)} \chi_{\pi}(r)
$$

We show in Lemma 26 that $S_{1 / 6}=\left|R_{D, 1 / 6}(\tau) D^{-1 / 3}\right|^{2}$. Moreover, if we denote

$$
T_{D}=R_{D, 1 / 6}(\tau / 3) \bar{\pi}^{-2 / 3} \omega^{k_{0}},
$$

for a cubic root of unity $\omega^{k_{0}}$, then $S_{1 / 6}=\left|T_{D, 1 / 6}(\tau)\right|^{2}$.

We show in Lemma 30 that $T_{D}=\frac{1}{\varphi(D)} \operatorname{Tr}_{H_{\mathscr{O}} / K} \frac{\theta_{1,1 / 6}(\tau)}{\theta_{0}(\tau)} \bar{\pi}^{-2 / 3} \omega^{k_{0}}$ and that $T_{D} \in K$. Furthermore, we show in proposition 25 that $T_{D}=(-1)^{\sigma(D)} \overline{T_{D}}$ and thus $T_{D} \in \mathbb{Q}$ or $T_{D} / \sqrt{-3} \in \mathbb{Q}$ and thus

$$
S_{D}=\frac{(-1)^{\sigma(D)}}{3 c_{3 D}} T_{D}^{2}
$$

Moreover, in Sect. 4.5 we show that $3 c_{3 D} S_{D}$ is an integer, hence $T_{D} / 3 \in \mathbb{Z}$ for $\sigma(D)$ even and $T_{D} / \sqrt{-3} \in \mathbb{Z}$ for $\sigma(D)$ odd.

Finally, for $D$ a product of split primes, we have $S_{D} \neq 0$ only for $D \equiv 1 \bmod 9$. In this case we can compute the Tamagawa numbers to be $c_{3 D}=3^{1+\sigma(D)}$ using Tate's algorithm (see [28, Chapter IV.9]). Thus we have:

$$
S_{D}= \begin{cases}\left(T_{D} / 3^{\sigma(D) / 2+1}\right)^{2}, & \text { for } \sigma(D) \text { even } \\ \left(\left(T_{D} / \sqrt{-3}\right) / 3^{(\sigma(D)+1) / 2}\right)^{2}, & \text { for } \sigma(D) \text { odd }\end{cases}
$$

Hence $S_{D}$ is an integer square up to an even power of 3 and this finishes the proof of Theorem 14.

For the reader's convenience, we summarize the notations used in the whole chapter in the table below: 


\begin{tabular}{|c|c|}
\hline Sums & Theta functions and ratios \\
\hline$S_{D}=\frac{1}{3} \sum_{[\mathscr{A}] \in \operatorname{Cl}\left(\mathscr{O}_{3 D_{0}}\right)} \frac{\Theta_{K}\left(D_{0} \tau_{\mathscr{A}}\right)}{\Theta_{K}\left(\tau_{\mathscr{A}}\right)} \chi_{D}(\mathscr{A}) D^{1 / 3}$ & $\Theta_{K}(z)=\sum_{a, b \in \mathbb{Z}} e^{2 \pi i z\left(a^{2}+b^{2}-a b\right)}$ \\
\hline$S_{\mu}=\frac{2}{3} \sum_{[\mathscr{A}] \in \operatorname{Cl}\left(\mathscr{O}_{3 D}\right)} \frac{\Theta_{\mu}\left(D \tau_{\mathscr{A}}\right)}{\Theta_{K}\left(\tau_{\mathscr{A}}\right)} \chi_{D}(\mathscr{A}) D^{1 / 3} t$ & $\left\{\begin{array}{l}\Theta_{1 / 2}(\tau)=\Theta_{K}(\tau / 3) \\
\Theta_{1 / 6}(\tau)=\frac{3}{2} \Theta_{K}(\tau)-\frac{1}{2} \Theta_{K}(\tau / 3)\end{array}\right.$ \\
\hline$R_{D, \mu}(z)=\sum_{r \in(\mathbb{Z} / D \mathbb{Z})} \times \frac{\theta_{r, \mu}(3 z)}{\theta_{0}(3 z)} \chi_{\pi}(r)$ & $\theta_{r, \mu}(z)=\sum_{n \in \mathbb{Z}}(-1)^{n} e^{\pi i\left(n+\frac{r}{D}-\frac{1}{2}\right)^{2} z}$ \\
\hline$=\sum_{r \in(\mathbb{Z} / D \mathbb{Z})^{r \equiv 1(6)}} \times F_{r, \mu}(z) \chi_{\pi}(r)$ & $\begin{array}{l}\theta_{0}(z)=\theta_{0,1 / 6}(z)=\eta(z / 3) \\
f_{r, \mu}(z)=\frac{\theta_{r, \mu}(z)}{\theta_{0}(z)}, F_{r, \mu}(z)=f_{r, \mu}(3 z)\end{array}$ \\
\hline$R^{(D), \mu}(z)=\sum_{\substack{r \in(\mathbb{Z} / D \mathbb{Z}) \\
r \equiv 1(6)}} \times F^{(r), \mu}(z) \chi_{\bar{\pi}}(r)$ & $\begin{array}{l}\theta^{(r), \mu}(z)=\sum_{n \in \mathbb{Z}} e^{\pi i(n-\mu)^{2} z}(-1)^{n} e^{2 \pi i n r / D} \\
F^{(r), \mu}(z)=\frac{\theta^{(r), \mu}(3 z)}{\theta_{0}(3 z)}\end{array}$ \\
\hline $\begin{array}{l}T_{D}=R_{D, 1 / 6}(\tau / 3) \bar{\pi}^{-2 / 3} \omega^{k_{0}} \\
M_{D}=R_{D, 1 / 6}(\tau / 3) \bar{\pi}^{-2 / 3}\end{array}$ & \\
\hline
\end{tabular}

\subsection{Factorization lemma}

As in the previous section, we write a primitive ideal $\mathscr{A}$ as a lattice $\mathscr{A}=\left[a, \frac{-b+\sqrt{-3}}{2}\right]_{\mathbb{Z}}$ for $a=\operatorname{Nm}(\mathscr{A})$ and $b^{2} \equiv-3 \bmod 4 a$. We also define the CM point $\tau_{\mathscr{A}}=\frac{-b+\sqrt{-3}}{2 a}$ corresponding to the $\mathbb{Z}$-lattice. We also denote by $k_{\mathscr{A}}$ the generator of $\mathscr{A}$ such that $k_{\mathscr{A}} \equiv 1$ (3) and we write the generator in the form $k_{\mathscr{A}}=n_{a} a+m_{a} \tau_{\mathscr{A}}$.

We start by recalling the Factorization Formula of Rodriguez-Villegas and Zagier from [23]. It is used to decompose theta functions of weight 1 evaluated at CM points as sums of products of theta functions of weight $1 / 2$ also evaluated at CM points.

Below we recall from [23, Theorem, page 7] for the simplified case of $\alpha=p=0$ :

Theorem 15 (Factorization formula) For $a \in \mathbb{Z}_{>0}, \mu, v \in \mathbb{Q}, z=x+y i \in \mathbb{C}$, we have:

$$
\sum_{m, n \in \mathbb{Z}} e^{2 \pi i(m v+n \mu)} e^{\pi\left(i m n-\frac{|m z-n|^{2}}{2 y}\right) / a}=\sqrt{2 a y} \theta\left[\begin{array}{c}
a \mu \\
v
\end{array}\right]\left(a^{-1} z\right) \cdot \theta\left[\begin{array}{c}
\mu \\
-a v
\end{array}\right](-a \bar{z}),
$$

where $\theta\left[\begin{array}{c}\mu \\ v\end{array}\right](z)=\sum_{n \in \mathbb{Z}+\mu} e^{\pi i n^{2} z+2 \pi i v n}$ is a theta function of weight $1 / 2$.

A direct application of this is the following:

Lemma 16 With notation as above, we have:

$$
\sum_{r \in \mathbb{Z} / D \mathbb{Z}} \frac{\sqrt{2 a y}}{\sqrt{D}} \theta\left[\begin{array}{c}
a \mu+\frac{a r}{D} \\
v
\end{array}\right]\left(D \frac{z}{a}\right) \theta\left[\begin{array}{c}
\mu+\frac{r}{D} \\
-a v
\end{array}\right](-a D \bar{z})=\sum_{m, n \in \mathbb{Z}} e^{2 \pi i(m v+n D \mu)} e^{\pi\left(m n i-\frac{|n-m z|^{2}}{2 y}\right) \frac{D}{a}}
$$


Proof Plugging in $\mu+\frac{r}{D}$ for $\mu$ and $D z$ for $z$ in (15), and summing up for $r$ in $\mathbb{Z} / D \mathbb{Z}$, we get:

$$
\begin{aligned}
\sum_{r \in \mathbb{Z} / D \mathbb{Z}} & \sqrt{2 a D y} \theta\left[\begin{array}{c}
a \mu+\frac{a r}{D} \\
v
\end{array}\right]\left(\frac{D z}{a}\right) \theta\left[\begin{array}{c}
\mu+\frac{r}{D} \\
-a v
\end{array}\right](-a \overline{D z})= \\
= & \sum_{r \in \mathbb{Z} / D \mathbb{Z}} \sum_{m, n \in \mathbb{Z}} e^{2 \pi i(m v+n \mu+n r / D)} e^{\pi\left(m n i-\frac{|n-m D z|^{2}}{2 D y}\right) \frac{1}{a}} .
\end{aligned}
$$

Exchanging the two sums on the RHS we get

$$
\sum_{m, n \in \mathbb{Z}} e^{2 \pi i(m v+n \mu)} e^{\pi\left(m n i-\frac{|n-m D z|^{2}}{2 D y}\right) \frac{1}{a}} \sum_{r \in \mathbb{Z} / D \mathbb{Z}} e^{2 \pi i n r / D} .
$$

The inner sum $\sum_{r \in \mathbb{Z} / D \mathbb{Z}} e^{2 \pi i n r / D}$ equals $D$ when $D \mid n$, and 0 otherwise, thus we are only summing over the integers $n$ that are multiples of $D$. Rewriting $n=D n^{\prime}$, after simplifying we get the result of the lemma.

Using the lemma above and the notation $\theta_{r, \mu}(z)=\sum_{n \in \mathbb{Z}}(-1)^{n} e^{\pi i\left(n+\frac{r}{D}-\mu\right)^{2} z}$, we show:

Proposition 17 For ideals $\mathscr{A}=\left[a, \frac{-b+\sqrt{-3}}{2}\right]_{\mathbb{Z}}, \mathscr{A}_{1}=\left[a_{1}, \frac{-b+\sqrt{-3}}{2}\right]_{\mathbb{Z}}$ and $b$ such that $b^{2} \equiv-3 \bmod 4 D^{2} a^{2} a_{1}$, we have:

$$
\Theta_{\mu}\left(D \tau_{\mathscr{A}}\right)=\frac{\sqrt[4]{3} e^{\pi i(a-1) / 6}}{D \sqrt{a_{1}}} \sum_{r \in \mathbb{Z} / D \mathbb{Z}} \theta_{a r, \mu}\left(\tau_{\mathscr{A}^{2} \mathscr{A}_{1}}\right) \overline{\theta_{r, \mu}\left(\tau_{\mathscr{A}_{1}}\right)}
$$

Proof We apply Lemma 16 for $\mu=-1 / 6$ and $v=1 / 2, D$ odd, $z=\frac{-b+\sqrt{-3}}{2 D a a_{1}}$. It is easy to see on the LHS of the equation that we have $\theta\left[\begin{array}{c}-1 / 6+\frac{r}{D} \\ -a / 2\end{array}\right](z)=$ $e^{-a \pi i r / D} e^{a \pi i / 6} \theta_{r, \mu}(z)$, and, as $a \equiv 1(\bmod 6)$, also $\theta\left[\begin{array}{c}-a / 6+\frac{a r}{D} \\ 1 / 2\end{array}\right](z)=e^{\pi i a r / D}$ $e^{-\pi i / 6} \theta_{a r, \mu}(z)$. Moreover, since $D \equiv 1(\bmod 6)$ we simplify the term $e^{-2 \pi i n D / 6}=$ $e^{-2 \pi i n / 6}$ and we can also compute $\frac{\sqrt{2 a y}}{\sqrt{D}}=\frac{\sqrt[4]{3}}{D \sqrt{a_{1}}}$. We get:

$\frac{\sqrt[4]{3}}{D \sqrt{a_{1}} e^{\pi i \frac{a-1}{6}}} \sum_{r \in \mathbb{Z} / D \mathbb{Z}} \theta_{a r, \mu}\left(\tau_{\mathscr{A}^{2} \mathscr{A}_{1}}\right) \overline{\theta_{r, \mu}\left(\tau_{\mathscr{A}_{1}}\right)}=\sum_{m, n \in \mathbb{Z}} e^{2 \pi i\left(\frac{m}{2}-\frac{n}{6}\right)} e^{\pi\left(m n i-\frac{\left|n D a a_{1}-m \frac{-b+\sqrt{-3}}{2}\right|^{2}}{D a a_{1} \sqrt{3}}\right) \frac{D}{a}}$.

Now we only have to show that the RHS equals $\Theta_{\mu}\left(D \tau_{\mathscr{A}}\right)$. We claim:

$$
e^{2 \pi i(m / 2+n / 2)} e^{\pi\left(m n i-\frac{\left|n a a_{1} D-m \frac{-b+\sqrt{-3}}{2}\right|^{2}}{a a_{1} D \sqrt{3}}\right) \frac{D}{a}}=e^{2 \pi i \frac{\left|n a a_{1} D-m \frac{-b+\sqrt{-3}}{2}\right|^{2}}{a a_{1} D} D \frac{-b+\sqrt{-3}}{6 a}} .
$$


Since the absolute values of the two sides already agree, we only need to show that the arguments agree as well, meaning $2 \pi i\left(\frac{m}{2}+\frac{n}{2}+\frac{D m n}{2 a}\right) \equiv-2 \pi i \frac{\left|n a a_{1} D-m \frac{-b+\sqrt{-3}}{2}\right|^{2}}{a a_{1} D}$ $D \frac{b}{6 a} \bmod 2 \pi i \mathbb{Z}$. This is equivalent to showing that

$$
\left(\frac{m^{2}}{2}+\frac{n^{2}}{2}\right)-\left(\frac{m^{2}}{2} D b \frac{\left(b^{2}+3\right)}{12 a^{2} a_{1}}-D m n \frac{\left(b^{2}+3\right)}{6 a}+D \frac{n^{2}}{2} \frac{b}{3}\right) \in \mathbb{Z},
$$

and this follows easily from the conditions on $a, a_{1}, b$ and $D$.

Finally, we claim that $\Theta_{\mu}(z)=\sum_{m, n \in \mathbb{Z}} e^{2 \pi i n(\mu+1 / 2)} e^{2 \pi i \frac{\left|m \cdot \frac{b+\sqrt{-3}}{2}+n a a_{1}\right|^{2}}{a a_{1}}} z$, which would finish the proof. This is immediate for $\mu=1 / 2$. For $\mu=1 / 6$ denote

$$
E_{*, k}(z)=\sum_{\substack{m, n \in \mathbb{Z} \\ n \equiv k(3)}} e^{2 \pi i n / 3} e^{2 \pi i \frac{\left|m \cdot \frac{b+\sqrt{-3}}{2}+n a a_{1}\right|^{2}}{a a_{1}}} z
$$

Then we can write

$$
\sum_{m, n \in \mathbb{Z}} e^{2 \pi i n / 3} e^{2 \pi i \frac{\left|m \cdot \frac{b+\sqrt{-3}}{2}+n a a_{1}\right|^{2}}{a a_{1}} z}=E_{*, 0}(z)+\omega E_{*, 1}(z)+\omega^{2} E_{*, 2}(z) .
$$

Note that $E_{*, 0}(z)=\Theta_{K}(3 z)$ and $E_{*, 1}=E_{*, 2}$, as we can change $n \rightarrow-n, m \rightarrow-m$ in the Fourier expansion. Thus we get on the RHS the term $\Theta(3 z)+\left(\omega+\omega^{2}\right) E_{*, 1}(z)=$ $\Theta(3 z)-E_{*, 1}(z)$. Furthermore $\Theta(z)=E_{*, 1}(z)+E_{*, 2}(z)+E_{*, 0}(z)$, thus we get $E_{*, 1}(z)=\frac{1}{2}(\Theta(z)-\Theta(3 z))$. Plugging in $E_{*, 1}(z)$ above we get the result of the proposition.

A particular case of Lemma 17 is for $D=1$. As $\Theta\left(\tau_{A} / 3\right)=0$ from Lemma 43 from the Appendix, we get:

Corollary 18 For $b^{2} \equiv-3 \bmod 12 a^{2} a_{1}$ and $\mathscr{A}, \mathscr{A}_{1}$ as above, we have

$$
\Theta_{K}\left(\tau_{\mathscr{A}}\right)=\frac{2}{3} \frac{\sqrt[4]{3}}{\sqrt{a_{1}}} e^{\pi i(a-1) \frac{1}{6}} \theta_{0}\left(\tau_{\mathscr{A}^{2} \mathscr{A}_{1}}\right) \overline{\theta_{0}\left(\tau_{\mathscr{A}_{1}}\right)}
$$

Let:

$$
f_{r, \mu}(z)=\frac{\theta_{r, \mu}(z)}{\theta_{0}(z)}
$$

Taking the ratios of the theta functions in Proposition 17 and Corollary 18 we get:

Corollary 19 Under the same conditions as above, we have:

$$
\frac{\Theta_{\mu}\left(D \tau_{\mathscr{A}}\right)}{\Theta\left(\tau_{\mathscr{A}}\right)}=\frac{3 / 2}{D} \sum_{r \in \mathbb{Z} / D \mathbb{Z}} f_{a r, \mu}\left(\tau_{\mathscr{A}^{2} \mathscr{A}_{1}}\right) \overline{f_{r, \mu}\left(\tau_{\mathscr{A}_{1}}\right)}
$$


We are interested in the Galois conjugates of $f_{r, \mu}(\tau)$ for $\tau=\frac{-b+\sqrt{-3}}{2}$ such that $b^{2} \equiv-3 \bmod 12 D^{2}$. For $\mathscr{A}=\left[a, \frac{-b+\sqrt{-3}}{2}\right]_{\mathbb{Z}}$ a primitive ideal and $k_{\mathscr{A}} \equiv 1 \bmod 3$ its generator, we write $k_{\mathscr{A}}$ in the form $k_{\mathscr{A}}=n_{a} a+m_{a} \frac{-b+\sqrt{-3}}{2}$ with $3 \mid m_{a}$ and $n_{a} \equiv 1(3)$.

In Sect. 5.2, we compute the Galois conjugates of $f_{r, \mu}(\tau)$ using Shimura reciprocity's law in two ways. We first show in Lemma 38 that

$$
\left(f_{r, \mu}(\tau)\right)^{\sigma_{\mathscr{A}}^{-1}}=f_{r, \mu}\left(\tau_{\mathscr{A}}\right)
$$

where $\sigma_{\mathscr{A}}$ is the Galois action corresponding to the ideal $\mathscr{A}$ via the Artin map. With the same notation, we show in Proposition 40 that

$$
f_{r, \mu}(\tau)^{\sigma_{\mathscr{A}}^{-1}}=f_{n_{a}^{\prime} r, \mu}(\tau)
$$

where $n_{a}^{\prime} \equiv n_{a}(3 D)$ with $n_{a}^{\prime}$ odd. Thus we get the following lemma:

Lemma 20 For an ideal $\mathscr{A}=\left[a, \frac{-b+\sqrt{-3}}{2}\right]$ generated by $n_{a} a+m_{a} \frac{-b+\sqrt{-3}}{2}$ such that $m_{a} \equiv 0 \bmod 3, n_{a} \equiv 1 \bmod 3$ and $b^{2} \equiv-3 \bmod 12 a D^{2}$, we have:

$$
f_{r, \mu}(\tau)^{\sigma_{\mathscr{A}}^{-1}}=f_{r, \mu}\left(\tau_{\mathscr{A}}\right)=f_{n_{a}^{\prime} r, \mu}(\tau)
$$

for $n_{a}^{\prime} \equiv n_{a}(3 D)$ with $n_{a}^{\prime}$ odd.

Using the lemma above, we can rewrite Corollary 19:

Corollary 21 Under the same conditions as above, for $\mathscr{A}=\left(n_{a} a+m_{a} \frac{-b+\sqrt{-3}}{2}\right)$, $\mathscr{A}_{1}=\left(n_{a_{1}} a_{1}+m_{a_{1}} \frac{-b+\sqrt{-3}}{2}\right)$ with $b^{2} \equiv-3 \bmod 12 a^{2} a_{1} D^{2}$, we have:

$$
\frac{\Theta_{\mu}\left(D \tau_{\mathscr{A}}\right)}{\Theta\left(\tau_{\mathscr{A}}\right)}=\frac{3 / 2}{D} \sum_{r \in \mathbb{Z} / D \mathbb{Z}} f_{n_{a}^{\prime 2} n_{a_{1}}^{\prime} a r, \mu} \overline{f_{n_{a_{1}^{\prime}} r, \mu}(\tau)},
$$

where $n_{a}^{\prime} \equiv n_{a}(3 D), n_{a_{1}}^{\prime} \equiv n_{a_{1}}(3 D)$ and $n_{a}^{\prime}, n_{a_{1}}^{\prime}$ odd.

\section{2 $S_{D}$ as an absolute value}

In the following we will use Corollary 21 for a choice of representative ideals for the classes of the ring class group $\mathrm{Cl}\left(\mathscr{O}_{3 D}\right)$. We show first:

Proposition 22 For $\tau=\frac{-b+\sqrt{-3}}{2}$ such that $b^{2} \equiv-3 \bmod 12 D^{2}$ and $\pi \equiv 1 \bmod 3$ an element of norm of $D$ such that $(\pi)^{2}$ divides $\left(\frac{-b+\sqrt{-3}}{2}\right)$, we have:

$$
S_{\mu}=D^{-2 / 3}\left|\sum_{\substack{s \in(\mathbb{Z} / D \mathbb{Z})^{\times} \\ s \equiv 1(6)}} f_{s, \mu}(\tau) \chi_{\pi}(s)\right|^{2} .
$$


Proof The structure of the ring class group of conductor $3 D$ for $D=\prod_{p_{i} \equiv 1 \bmod 3} p_{i}$ is given by $\mathrm{Cl}\left(\mathscr{O}_{3 D}\right) \cong(\mathbb{Z} / D \mathbb{Z})^{\times}$(see for example Cox [5]). We will choose as representatives for the classes of $\operatorname{Cl}\left(\mathscr{O}_{3 D}\right)$ ideals $\mathscr{A}_{s}$ such that $\mathrm{Nm} \mathscr{A}_{S} \equiv s \bmod D$. For $b$ fixed, $b^{2} \equiv-3 \bmod 12 D$, we take:

$$
\mathscr{A}_{s}=\left(n_{s} a_{s}+m_{s} \frac{-b+\sqrt{-3}}{2}\right)
$$

where $a_{s}=\operatorname{Nm}\left(\mathscr{A}_{s}\right) \equiv s \bmod D, n_{s} \equiv 1 \bmod 3 D, m_{s} \equiv 0 \bmod 3$. Note that this gives us $m \equiv b^{-1}(s-1) \bmod 3 D$. Moreover, it is easy to check that the ideals $\mathscr{A}_{s}$ for $s \in(\mathbb{Z} / D \mathbb{Z})^{\times}$are in different classes in $\mathrm{Cl}\left(\mathscr{O}_{3 D}\right)$.

We take as before $\pi$ the element of norm $D$ such that $(\pi)^{2}$ divides the ideal $(\tau)=$ $\left(\frac{-b+\sqrt{-3}}{2}\right)$. Then note that $\chi_{D}(\alpha)=\chi_{\pi}\left(\frac{\alpha}{\bar{\alpha}}\right)=\chi_{\pi}\left(\frac{\alpha^{2}}{|\alpha|^{2}}\right)$. As $b \equiv \sqrt{-3} \bmod \pi$, we get $\alpha_{s}=n_{s} a_{s}+m_{s} \frac{-b+\sqrt{-3}}{2} \equiv s \bmod \pi$ and thus $\chi_{\pi}\left(\left(\alpha_{s}\right)\right)=\chi_{\pi}\left(s^{2} / s\right)=\chi_{\pi}(s)$.

Taking representatives $s \in \mathbb{Z} / D \mathbb{Z}, s \equiv 1 \bmod 6$, we get $m_{s} \equiv 0 \bmod 6$ and $n_{s} \equiv 1 \bmod 6$. Summing up over $r \in \mathbb{Z} / D \mathbb{Z}$ with $r \equiv 1 \bmod 6$ and taking $\mathscr{A}_{1}=(1)$ in Corollary 21, we get:

$$
\frac{\Theta_{\mu}\left(D \tau_{\mathscr{A}_{s}}\right)}{\Theta\left(\tau_{\mathscr{A}_{s}}\right)}=\frac{3 / 2}{D} \sum_{\substack{r \in \mathbb{Z} / D \mathbb{Z} \\ r \equiv 1(6)}} f_{s r, \mu}(\tau) \overline{f_{r, \mu}(\tau)},
$$

Summing up for all $\left\{s \in(\mathbb{Z} / D \mathbb{Z})^{\times}, s \equiv 1(6)\right\}$ and rearranging the terms, we get:

$$
S_{\mu}=D^{-2 / 3} \sum_{\substack{s \in(\mathbb{Z} / D \mathbb{Z})^{\times} \\ s \equiv 1(6)}} \sum_{\substack{r \in \mathbb{Z} / D \mathbb{Z}, r \equiv 1(6)}} f_{s r, \mu}(\tau) \chi_{\pi}(r s) \cdot \overline{f_{r, \mu}(\tau) \chi_{\pi}(r)} .
$$

Finally, we will further modify the sums on the RHS in order to sum up over $r \in(\mathbb{Z} / D \mathbb{Z})^{\times}$as well. In order to emphasize the dependence of $\theta_{r, \mu}$ on $D$ we will use only for the current proof the notation

$$
f_{r / D}(z)=\frac{\theta_{r, \mu}(z)}{\theta_{0}(z)}=\frac{\sum_{n \in \mathbb{Z}} e^{\pi i\left(n+\frac{r}{D}-\mu\right)^{2} z}(-1)^{n}}{\theta_{0}(z)} .
$$

Moreover, for $p_{i_{1}} \ldots p_{i_{k}} \mid D$, denote:

$$
S_{p_{i_{1}} \ldots p_{i_{k}}}=\sum_{\substack{s \in(\mathbb{Z} / D \mathbb{Z})^{\times} \\ s \equiv 1(6)}} \chi_{D}\left(\mathscr{A}_{S}\right) D^{-2 / 3} \sum_{\substack{r \in \mathbb{Z} / D \mathbb{Z} \\ r \equiv 1(6) \\ p_{i_{1}} \ldots p_{i_{k}} \mid r}} f_{s r / D}(\tau) \overline{f_{r / D}(\tau)}
$$


We claim that for $k \geq 1$ we have $S_{p_{i_{1}} \ldots p_{i_{k}}}=0$. Note that we can rewrite

$$
\begin{aligned}
S_{\mu}= & \sum_{p_{i} \mid D} S_{p_{i}}-\sum_{p_{i} p_{j} \mid D} S_{p_{i} p_{j}}+\cdots+(-1)^{n-1} S_{p_{1}, \ldots, p_{n}} \\
& +\sum_{\substack{s, r \in(\mathbb{Z} / D \mathbb{Z})^{\times} \\
s \equiv r \equiv 1(\bmod 6)}} f_{s r / D}(\tau) \overline{f_{r / D}(\tau)} \chi_{D}\left(\mathscr{A}_{S}\right) D^{-2 / 3},
\end{aligned}
$$

thus showing $S_{p_{i_{1}} \ldots p_{i_{k}}}=0$ for $k \geq 1$ proves our result.

To see that $S_{p_{i_{1}} \ldots p_{i_{k}}}=0$, let $D^{\prime}=D /\left(p_{i_{1}} \ldots p_{i_{k}}\right)$. We recognize each of the inner sums $\sum_{\substack{r^{\prime} \in \mathbb{Z} / D^{\prime} \mathbb{Z} \\ r \equiv 1(\bmod 6)}} f_{s r^{\prime} / D^{\prime}}(\tau) \overline{f_{r^{\prime} / D^{\prime}}(\tau)}$ of $S_{p_{i_{1}} \ldots p_{i_{k}}}$ to be equal to $\frac{D^{\prime}}{3 / 2} \frac{\Theta_{\mu}\left(D^{\prime} \tau_{\mathscr{A} s}\right)}{\Theta\left(\tau_{\mathscr{A}}\right)}$ from (18) for $D=D^{\prime}$.

Denote $m=D / D^{\prime}$. From the properties of the cubic character, we have $\chi_{D}=$ $\chi_{m} \chi_{D^{\prime}}$. Moreover, from our choice of ideals, we have $\frac{\Theta_{\mu}\left(D^{\prime} \tau_{\mathscr{A} S}\right)}{\Theta\left(\tau_{\mathscr{A} s}\right)}=\frac{\Theta_{\mu}\left(D^{\prime} \tau_{\mathscr{A}}{ }_{s^{\prime}}\right)}{\Theta\left(\tau_{\mathscr{A}} s_{s^{\prime}}\right)}$ for $s \equiv s^{\prime} \bmod 3 D^{\prime}$, as $\mathscr{A}_{s}$ and $\mathscr{A}_{s^{\prime}}$ are in the same class in $\operatorname{Cl}\left(\mathscr{O}_{3 D^{\prime}}\right)$. Then we can rewrite the sum as:

$$
S_{p_{i_{1}} \ldots p_{i_{k}}}=\sum_{\substack{s^{\prime} \in\left(\mathbb{Z} / D^{\prime} \mathbb{Z}\right)^{\times}, s^{\prime} \equiv 1(\bmod 6)}} \frac{D^{\prime}}{3 / 2} \frac{\Theta_{\mu}\left(D^{\prime} \tau_{\mathscr{A}_{s}}\right)}{\Theta\left(\tau_{\mathscr{A}_{s}}\right)} \chi_{D^{\prime}}\left(\mathscr{A}_{s}\right) \sum_{\substack{s \in(\mathbb{Z} / D \mathbb{Z})^{\times}, s, s^{\prime} \equiv 1(\bmod 6) \\ s \equiv s^{\prime}\left(\bmod D^{\prime}\right)}} \chi_{m}\left(\mathscr{A}_{s}\right)
$$

In the inner sum we are summing over $s$ modulo $m$ for all $s$ in $(\mathbb{Z} / m \mathbb{Z})^{\times}$. Moreover, $\chi_{m}\left(\mathscr{A}_{S}\right)$ is a nontrivial character as a function of $s$, as $m^{1 / 3} \chi_{m}\left(\mathscr{A}_{s}\right)=\left(m^{1 / 3}\right)^{\sigma_{\mathscr{A}} s}=$ $m^{1 / 3}$ for all $\mathscr{A}_{s}$ iff $m^{1 / 3} \in K$. As we are summing a non-trivial character over a group, the sum is 0 . This finishes the proof.

Using the above proposition now it is easy to see:

Corollary $23 S_{D}=\frac{1}{3 c_{3 D}} S_{1 / 6}$ and $S_{1 / 2}=0$.

Proof As $3 c_{3 D} S_{D}=S_{1 / 6}+1 / 2 S_{1 / 2}$, if $S_{1 / 2}=0$ we have $3 S_{D} c_{3 D}=S_{1 / 6}$. Thus it is enough to show $S_{1 / 2}=0$. More precisely we will show that

$$
U(z)=\sum_{\substack{r \in(\mathbb{Z} / D \mathbb{Z})^{\times} \\ r \equiv 1(6)}} \theta_{r, 1 / 2}(z) \chi_{\pi}(r)
$$

equals 0 for any $z$, in particular for $z=\tau$. Since we showed that $S_{1 / 2}=D^{-2 / 3}|R(\tau)|^{2}$ in Proposition 22, we get $S_{1 / 2}=0$.

To show $U(z)=0$, note that $\theta_{r, 1 / 2}(z)=-\theta_{2 D-r, 1 / 2}(z)$, while $\chi_{\pi}(2 D-r)=$ $\chi_{\pi}(r)$. As both $r, 2 D-r \equiv 1 \bmod 6$ and $D$ odd, the terms cancel each other out in the sum and we get $U(z)=0$. 
Finally, from Proposition 22 and Corollary 23 we get:

Proposition 24 For $\tau=\frac{-b+\sqrt{-3}}{2}$ such that $b^{2} \equiv-3 \bmod 12 D^{2}$, we have $S_{D}=$ $\frac{1}{3 c_{3 D}} S_{1 / 6}$ and

$$
S_{D}=\frac{D^{-2 / 3}}{3 c_{3 D}}\left|\sum_{\substack{s \in(\mathbb{Z} / D \mathbb{Z})^{\times} \\ s \equiv 1(6)}} f_{s, 1 / 6}(\tau) \chi_{\pi}(s)\right|^{2} .
$$

\section{3 $S_{D}$ as a square}

In the following we will rewrite Proposition 24 so that we get a square. Define $F_{r, \mu}(z)=f_{r, \mu}(3 z)$ and take:

$$
R_{D, \mu}(z)=\sum_{\substack{r \in(\mathbb{Z} / D \mathbb{Z})^{\times} \\ r \equiv 1(6)}} F_{r, \mu}(z) \chi_{\pi}(r)
$$

With this notation, we have showed in Proposition 22 that $S_{1 / 6}=\left|R_{D, 1 / 6}(\tau / 3) D^{-1 / 3}\right|^{2}$. One can show that $R_{D, 1 / 6}^{3}(\tau / 3) \in K$ and actually $R_{D, 1 / 6}(\tau / 3)$ is really close to being an integer. We will show in this section the following:

Proposition 25 For $\sigma(D)$ the number of prime divisors of $D$, we have:

$$
S_{D}=\frac{(-1)^{\sigma(D)}}{3 c_{3 D}} T_{D}^{2},
$$

where $T_{D}=R_{D, 1 / 6}(\tau / 3) \bar{\pi}^{-2 / 3} \omega^{k_{0}}$ and $T_{D}=(-1)^{\sigma(D)} \overline{T_{D}}$ and thus $T_{D}$ is real or purely imaginary. Here $\omega^{k_{0}}$ is the unique cube root that makes $T_{D}$ real or purely imaginary.

We are going to show first in Lemma 26 that $R_{D, 1 / 6}(\tau)$ and $R_{D, 1 / 6}(\tau / 3)$ differ only by a cubic root of unity $\omega^{k}$, and thus $S_{1 / 6}=\left|R_{D, 1 / 6}(\tau) D^{-1 / 3}\right|^{2}$. In Proposition 27 we show that $R_{D, 1 / 6}(\tau)=(-1)^{\sigma(D)} \frac{\pi^{2 / 3}}{\pi^{2 / 3}} \overline{R_{D, 1 / 6}(\tau)} \omega^{k^{\prime}}$.

Defining $T_{D}=R_{D, 1 / 6}(\tau / 3) \bar{\pi}^{-2 / 3} \omega^{k_{0}}$ for $k_{0}=k+k^{\prime}$, this is equivalent to $T_{D}=$ $(-1)^{\sigma(D)} \overline{T_{D}}$ and thus

$$
S_{1 / 6}=(-1)^{\sigma(D)} T_{D}^{2}
$$

which is the result of Proposition 25 above.

\subsubsection{Relating $S_{D}$ to $R_{D, 1 / 6}(\tau)$}

We will first show that $S_{1 / 6}=\left|R_{D, 1 / 6}(\tau) D^{-1 / 3}\right|^{2}$ in Lemma 26. Define the theta function $\theta^{(r), \mu}(z)=\sum_{n \in \mathbb{Z}} e^{\pi i(n-\mu)^{2} z}(-1)^{n} e^{2 \pi i n r / D}$ and the ratio $F^{(r), \mu}(z)=\frac{\theta^{(r), \mu}(3 z)}{\theta_{0}(3 z)}$. 
We introduce this notation, as we will use the transformation mentioned in Lemma 48 in the Appendix:

$$
\theta_{r, 1 / 6}(3 z)=(-1)^{r} \frac{(-1)^{\frac{D-1}{6}} \omega}{\sqrt{-3} \sqrt{-i z}}\left(\theta^{(3 r), 1 / 6}\left(-\frac{3}{z}\right)-\omega \theta^{(-3 r), 1 / 6}\left(-\frac{3}{z}\right)-\omega^{2} \theta^{(-3 r), 1 / 2}\left(-\frac{3}{z}\right)\right) .
$$

Using also $\theta_{0}(3 z)=\frac{1}{\sqrt{-i z}} \theta_{0}(-3 / z)$ and taking the ratio with (19) we get:

$$
F_{r, 1 / 6}(z)=(-1)^{r} \frac{(-1)^{\frac{D-1}{6}} \omega}{\sqrt{-3}}\left(F^{(3 r), 1 / 6}\left(-\frac{1}{z}\right)-\omega F^{(-3 r), 1 / 6}\left(-\frac{1}{z}\right)-\omega^{2} F^{(-3 r), 1 / 2}\left(-\frac{1}{z}\right)\right) .
$$

Then, using (20), we are ready to show:

Lemma 26 For $\tau=\frac{-b+\sqrt{-3}}{2}$ such that $b^{2} \equiv-3 \bmod 12 D^{2}$, we have:

$$
R_{D, 1 / 6}(\tau)=\omega^{k} R_{D, 1 / 6}(\tau / 3)
$$

where $\omega^{k}$ is a cubic root of unity. Furthermore, this implies $S_{1 / 6}=\left|R_{D, 1 / 6}(\tau) D^{-1 / 3}\right|^{2}$.

Proof Let $b^{\prime} \equiv b \bmod 4 D^{2}$, and $b^{\prime} \neq \equiv 0 \bmod 3$. Without loss of generality we can actually pick $b, b^{\prime}$ such that $\left(b^{2}+3\right) / 12 D^{2}$ and $\left(b^{\prime 2}+3\right) / 4 D^{2}$ are prime to $3 D$. Let $\pi \equiv 1(3)$ be an element of norm $D$ such that $(\pi)^{2}$ divides $(\tau)$. Then we can find ideals $\mathscr{A}, \mathscr{A}^{\prime}$ prime to $3 D$ such that:

$$
(\sqrt{-3})(\pi)^{2} \mathscr{A}=\left(\frac{-b+\sqrt{-3}}{2}\right),(\pi)^{2} \mathscr{A}^{\prime}=\left(\frac{-b^{\prime}+\sqrt{-3}}{2}\right) .
$$

We can write the generators $k_{\mathscr{A}}, k_{\mathscr{A}} \equiv 1 \bmod 3$ of $\mathscr{A}$ and $\mathscr{A}^{\prime}$, respectively, in the form $k_{a}=a n_{a}+m_{a} \frac{-b+\sqrt{-3}}{2}, k_{a^{\prime}}=a^{\prime} n_{a^{\prime}}+m_{a^{\prime}} \frac{-b+\sqrt{-3}}{2}$, where $m_{a}, m_{a}^{\prime} \equiv 0 \bmod 3$, and $n_{a}, n_{a}^{\prime} \equiv 1 \bmod 3$. Let $\tau_{\mathscr{A}}=\frac{-b+\sqrt{-3}}{2 a}, \tau_{\mathscr{A}^{\prime}}=\frac{-b^{\prime}+\sqrt{-3}}{2 a^{\prime}}$.

We are going to show that:

(i) $R_{D, 1 / 6}\left(\tau_{\mathscr{A}}\right)=R_{D, 1 / 6}\left(\tau_{\mathscr{A}} / 3\right)$

(ii) $R_{D, 1 / 6}\left(\tau_{\mathscr{A}} / 3\right)=\chi_{\bar{\pi}}\left(n_{a}\right) R_{D, 1 / 6}(\tau / 3)$

These two relations will imply:

(iii) $R_{D, 1 / 6}(\tau)=\chi_{\bar{\pi}}\left(n_{a}\right) \chi_{\pi}\left(n_{a^{\prime}}\right) R_{D, 1 / 6}(\tau / 3)$.

In order to show (i), note that it is enough to show that $F_{r, 1 / 6}\left(\tau_{\mathscr{A}^{\prime}}\right)=F_{r, 1 / 6}\left(\tau_{\mathscr{A}} / 3\right)$. We have $-1 /\left(\tau_{A} / 3\right)=-\bar{\tau} / D^{2}$ and $-1 / \tau_{\mathscr{A}}=-\bar{\tau} / D^{2}$ as well and we will use (20) for both $\tau_{\mathscr{A}} / 3$ and $\tau_{\mathscr{A}^{\prime}} / 3$. First for $z=\tau_{\mathscr{A}} / 3$ we get:

$$
F_{r, 1 / 6}\left(\frac{\tau_{\mathscr{A}}}{3}\right)=\frac{(-1)^{r+\frac{D-1}{6}} \omega}{\sqrt{-3}}\left(F^{(3 r), 1 / 6}\left(\frac{-\bar{\tau}}{D^{2}}\right)-\omega F^{(-3 r), 1 / 6}\left(\frac{-\bar{\tau}}{D^{2}}\right)-\omega^{2} F^{(3 r), 1 / 2}\left(\frac{-\bar{\tau}}{D^{2}}\right)\right) .
$$


Applying (20) also for $\tau_{\mathscr{A}}$, we get similarly

$F_{r, 1 / 6}\left(\tau_{\mathscr{A}^{\prime}}\right)=\frac{(-1)^{r+\frac{D-1}{6}} \omega}{\sqrt{-3}}\left(F^{(3 r), 1 / 6}\left(\frac{-\overline{\tau^{\prime}}}{D^{2}}\right)-\omega F^{(-3 r), 1 / 6}\left(\frac{-\overline{\tau^{\prime}}}{D^{2}}\right)-\omega^{2} F^{(3 r), 1 / 2}\left(\frac{-\overline{\tau^{\prime}}}{D^{2}}\right)\right)$,

where $\tau^{\prime}=\frac{-b^{\prime}+\sqrt{-3}}{2}$.

Finally, note that $F^{(s), 1 / 6}\left(z+8 D^{2}\right)=F^{(s), 1 / 6}(z)$, thus since $b \equiv b^{\prime} \bmod 8 D^{2}$ we also have $F^{(s), 1 / 6}\left(-\overline{\tau^{\prime}} / D^{2}\right)=F^{(s), 1 / 6}\left(-\bar{\tau} / D^{2}\right)$ for $s= \pm 3 r$. Similarly we have $F^{(-3 r), 1 / 2}\left(-\overline{\tau^{\prime}} / D^{2}\right)=F^{(-3 r), 1 / 2}\left(-\bar{\tau} / D^{2}\right)$, thus $F_{r, 1 / 6}\left(\tau_{\mathscr{A}} / 3\right)=F_{r, 1 / 6}\left(\tau_{\mathscr{A}^{\prime}}\right)$ as claimed.

To show (ii), as $f_{r, 1 / 6}(z)=F_{r, 1 / 6}(z / 3)$, note that from Lemma 20 we have $F_{r, 1 / 6}(\tau / 3)_{\mathscr{A}}^{-1}=F_{r, 1 / 6}\left(\tau_{\mathscr{A}} / 3\right)=F_{n_{a}^{\prime} r, 1 / 6}(\tau / 3)$ where $n_{a} \equiv n_{a}^{\prime} \bmod 3 D$ and $n_{a}^{\prime}$ odd. This further implies that $R_{D, 1 / 6}\left(\tau_{\mathscr{A}} / 3\right)=\chi_{\bar{\pi}}\left(n_{a}\right) R_{D, 1 / 6}(\tau / 3)$.

To show (iii), note that we are in the conditions of Lemma 38 from Sect. 5, as $F_{r, 1 / 6}$ is a modular function of level $18 D^{2}$. Then $F_{r, 1 / 6}(\tau)^{\sigma_{\mathscr{A}^{\prime}}^{-1}}=F_{r, 1 / 6}\left(\tau_{\mathscr{A}^{\prime}}\right)$ and thus we get $R_{D, 1 / 6}\left(\tau_{\mathscr{A}^{\prime}}\right)=\left(R_{D, 1 / 6}(\tau)\right)_{\mathscr{A}^{\prime}}^{-1}$.

We can rewrite this as $\left(R_{D, 1 / 6}\left(\tau_{\mathscr{A}^{\prime}}\right)\right)_{\mathscr{A}^{\prime}}=R_{D, 1 / 6}(\tau)$ and using $(i)$, we get

$R_{D, 1 / 6}(\tau)=R_{D, 1 / 6}\left(\tau_{\mathscr{A}} / 3\right)^{\sigma_{\mathscr{A}}}$. From (ii), this is $R_{D, 1 / 6}(\tau)=\chi_{\bar{\pi}}\left(n_{a}\right) R_{D, 1 / 6}(\tau / 3)^{\sigma_{\mathscr{A}^{\prime}}}$. Using Lemma 20, we have $F_{r, 1 / 6}(\tau / 3)^{\sigma_{\mathscr{A}^{\prime}}^{-1}}=F_{n_{a^{\prime}} r, 1 / 6}(\tau / 3)$, thus $R_{D, 1 / 6}(\tau / 3)^{\sigma_{\mathscr{A}}^{\prime}}=$ $\chi_{\pi}\left(n_{a^{\prime}}\right) R_{D, 1 / 6}(\tau / 3)$. Finally this implies

$R_{D, 1 / 6}(\tau)=\chi_{\bar{\pi}}\left(n_{a}\right) \chi_{\pi}\left(n_{a^{\prime}}\right) R_{D, 1 / 6}(\tau / 3)$ and we take $\omega^{k}=\chi_{\bar{\pi}}\left(n_{a}\right) \chi_{\pi}\left(n_{a^{\prime}}\right)$ to get the result.

\subsubsection{Relating $R_{D, 1 / 6}(\tau)$ to its complex conjugate}

Now we want to show that $R_{D, 1 / 6}(\tau)$ equals $\overline{R_{D, 1 / 6}(\tau)}$ up to a nice factor. As before we let $\tau=\frac{-b+\sqrt{-3}}{2}$ and $\pi$ such that $(\pi)^{2}$ divides $(\tau)$. We will show:

Proposition 27 For some cubic root of unity $\omega^{k^{\prime}}$, we have:

$$
R_{D, 1 / 6}(\tau)=(-1)^{\sigma(D)} \omega^{k^{\prime}} \frac{\bar{\pi}^{2 / 3}}{\pi^{2 / 3}} \overline{R_{D, 1 / 6}(\tau)}
$$

Using the notation $T_{D}=R_{D, 1 / 6}(\tau) \bar{\pi}^{-2 / 3} \omega^{k^{\prime}}$ this is equivalent to $T_{D}=(-1)^{\sigma(D)} \overline{T_{D}}$.

Note that we can think of $\omega^{k^{\prime}}$ as the unique root of unity which makes $R_{D, 1 / 6}(\tau) \omega^{k^{\prime}} \bar{\pi}^{-2 / 3}$ either real or purely imaginary. We actually give a formula for $\omega^{k^{\prime}}$ in the proof of Proposition 27.

We first define the linear combination:

$$
R^{(D), \mu}(z)=\sum_{\substack{r \in(\mathbb{Z} / D \mathbb{Z})^{\times} \\ r \equiv 1(6)}} F^{(r), \mu}(z) \chi_{\bar{\pi}}(r)
$$


Note that we use $\chi_{\pi}$, unlike in $R_{D, \mu}$.

We choose $b \equiv b^{\prime} \bmod 4 D^{2}$ such that $3 \nmid b^{\prime}$ and we can find $\mathscr{A}^{\prime}$ as in the proof of Lemma 26 such that $\mathscr{A}^{\prime}(\pi)^{2}=\left(\frac{-b^{\prime}+\sqrt{-3}}{2}\right)$. Then from the transformation (20) we have $F_{r, 1 / 6}\left(\tau_{\mathscr{A}^{\prime}}\right)=(-1)^{r} \frac{(-1)^{\frac{D-1}{6}} \omega}{\sqrt{-3}}\left(F^{(3 r), 1 / 6}\left(-\bar{\tau} / D^{2}\right)-\omega F^{(-3 r), 1 / 6}\left(-\bar{\tau} / D^{2}\right)-\right.$ $\left.\omega^{2} F^{(-3 r), 1 / 2}\left(-\bar{\tau} / D^{2}\right)\right)$. Writing the full linear combination for $r \in(\mathbb{Z} / D \mathbb{Z})^{\times}, r \equiv$ $1(6)$ and multiplying by $\chi_{\pi}(3)$, we get:

$$
(-1)^{\frac{D+1}{2}} \chi_{\pi}(3) R_{D, 1 / 6}\left(\tau_{\mathscr{A}^{\prime}}\right)=\overline{R^{(D), 1 / 6}\left(\tau / D^{2}\right)}-\omega^{2} \overline{R^{(D), 1 / 2}\left(\tau / D^{2}\right)} / \sqrt{-3} .
$$

Note that above we related $R_{D, 1 / 6}(\tau)$ to $\overline{R^{(D), \mu}}$ for $\mu \in\{1 / 2,1 / 6\}$. In order to show Proposition 27 we also want to relate $R^{(D), \mu}$ back to $R_{D, \mu}$, and we do that in the lemma below:

Lemma 28 We have the equality

$$
R^{(D), \mu}\left(\tau / D^{2}\right)=(-1)^{(D+1) / 2} \frac{G(\chi \bar{\pi})}{\bar{\pi}} R_{D, \mu}(\tau),
$$

where $G\left(\chi_{\bar{\pi}}\right)=\sum_{r \in(\mathbb{Z} / D \mathbb{Z})^{\times}} \chi_{\bar{\pi}}(r) e^{2 \pi i n r / D}$ is the Gauss sum corresponding to the character $\chi_{\bar{\pi}}$.

Proof Recall $R^{(D), \mu}\left(z / D^{2}\right)=\sum_{r \in(\mathbb{Z} / D \mathbb{Z})^{\times}} F^{(r), \mu}\left(z / D^{2}\right) \chi_{\bar{\pi}}(r)$, where $F^{(r), \mu}\left(z / D^{2}\right)=\frac{\theta^{(r), \mu}\left(3 z / D^{2}\right)}{\theta_{0}\left(3 z / D^{2}\right)}$. We show first that, for $r$ odd, we can rewrite the terms $\theta^{(r), \mu}\left(3 z / D^{2}\right)$ as:

$$
\theta^{(r), \mu}\left(3 z / D^{2}\right)=-\sum_{\substack{s \in(\mathbb{Z} / D \mathbb{Z}) \\ s \equiv 1(6)}} \theta_{s, \mu}(3 z) e^{2 \pi i r s / D}
$$

From the definition, we have $\theta^{(r), \mu}(3 z)=\sum_{n \in \mathbb{Z}} e^{\pi i(n-D \mu)^{2} 3 z}(-1)^{n} e^{2 \pi i n r / D}$. Choosing as before $s \in \mathbb{Z} / D \mathbb{Z}$ such that $s \equiv 1 \bmod 6$, we sum over all $n$ modulo $D$ :

$$
\theta^{(r), \mu}(3 z)=\sum_{\substack{s \in \mathbb{Z} / D \mathbb{Z} \\ s \equiv 1(6)}} \sum_{n \in \mathbb{Z}} e^{\pi i(D n+s-D \mu)^{2} 3 z}(-1)^{D n+s} e^{2 \pi i(D n+s) r / D} .
$$

We can rewrite this as $\theta^{(r), \mu}(3 z)=-\sum_{\substack{s \in \mathbb{Z} / D \mathbb{Z} \\ s \equiv 1(6)}} \theta_{s, \mu}\left(D^{2} 3 z\right) e^{2 \pi i s r / D}$ and changing $z \rightarrow z / 3 D^{2}$ we get (22).

Plugging in $z=\tau / D^{2}$ in (22) and dividing by $\theta_{0}(3 \tau)$ we have further

$$
\frac{\theta^{(r), \mu}\left(3 \tau / D^{2}\right)}{\theta_{0}(3 \tau)}=-\sum_{\substack{s \in \mathbb{Z} / D \mathbb{Z} \\ s \equiv 1(6)}} F_{s, \mu}(\tau) e^{2 \pi i r s / D} .
$$


Moreover from Lemma 44 in the Appendix:

$$
\frac{\theta_{0}\left(3 \tau / D^{2}\right)}{\theta_{0}(3 \tau)}=\frac{\eta\left(\tau / D^{2}\right)}{\eta(\tau)}=(-1)^{(D-1) / 6} \bar{\pi},
$$

thus we get:

$$
F^{(r), \mu}\left(\tau / D^{2}\right)=\frac{(-1)^{(D+1) / 2}}{\bar{\pi}} \sum_{\substack{s \in \mathbb{Z} / D \mathbb{Z} \\ s \equiv 1(6)}} F_{s, \mu}(\tau) e^{2 \pi i r s / D}
$$

Going back to the linear combination, we get

$$
R^{(D), \mu}\left(\tau / D^{2}\right)=-\frac{1}{\bar{\pi}} \sum_{\substack{r \in(\mathbb{Z} / D \mathbb{Z})^{\times} \\ r \equiv 1(6)}} \sum_{\substack{s \in \mathbb{Z} / D \mathbb{Z} \\ s \equiv 1(6)}} F_{s, \mu}(\tau) e^{2 \pi i s r / D} \chi_{\bar{\pi}}(r) .
$$

We switch the two sums and get:

$$
R^{(D), \mu}\left(z / D^{2}\right)=\frac{(-1)^{(D+1) / 2}}{\bar{\pi}} \sum_{\substack{(s \in \mathbb{Z} / D \mathbb{Z})^{\times} \\ s \equiv 1(6)}} F_{s, \mu}(\tau) \sum_{r \in(\mathbb{Z} / D \mathbb{Z})^{\times}} e^{2 \pi i s r / D} \chi_{\bar{\pi}}(r) .
$$

Note that if $\operatorname{gcd}(s, D)=D_{1}>1$, then the inner sum equals 0 . This is easily seen by writing $s=D_{1} s_{1}, D_{2}=D / D_{1}$, and rewriting

$$
\sum_{r \in(\mathbb{Z} / D \mathbb{Z})^{\times}} e^{2 \pi i s r / D} \chi_{\bar{\pi}}(r)=\chi_{\bar{\pi}}\left(s_{1}\right) \sum_{r \in(\mathbb{Z} / D \mathbb{Z})^{\times}} e^{2 \pi i r / D_{1}} \chi_{\bar{\pi}}(r)=\sum_{r \in\left(\mathbb{Z} / D_{2} \mathbb{Z}\right)^{\times}} \chi_{\bar{\pi}_{2}}(r) G\left(\chi_{\bar{\pi}_{1}}\right)=0,
$$

where $\pi=\pi_{1} \pi_{2}$ and $\operatorname{Nm}\left(\pi_{i}\right)=D_{i}$, for $i=1,2$. Thus we are left in our sum only with $s$ prime to $D$, and we can rewrite:

$$
R^{(D), \mu}\left(z / D^{2}\right)=\frac{(-1)^{(D+1) / 2}}{\bar{\pi}} \sum_{(s \in \mathbb{Z} / D \mathbb{Z})^{\times}} F_{s, \mu}(\tau) \chi_{\pi}(s) \sum_{r \in(\mathbb{Z} / D \mathbb{Z})^{\times}} e^{2 \pi i s r / D} \chi_{\bar{\pi}}(r s) .
$$

This is exactly $R^{(D), \mu}\left(\tau / D^{2}\right)=\frac{(-1)^{(D+1) / 2}}{\bar{\pi}} \sum_{\substack{(s \in \mathbb{Z} / D \mathbb{Z})^{\times} \\ s \equiv 1(6)}} F_{S, \mu}(\tau) \chi_{\pi}(s) G\left(\chi_{\bar{\pi}}\right)$, which equals $(-1)^{(D+1) / 2} \frac{G\left(\chi_{\bar{\pi}}\right)}{\bar{\pi}} R_{D, \mu}(\tau)$, and thus we get the result of our lemma.

Proof of Proposition 27 Recall from Eq. (21), we have

$$
(-1)^{\frac{D-1}{2}} \chi_{\pi}(3) R_{D, 1 / 6}\left(\tau_{\mathscr{A}^{\prime}}\right)=\overline{R^{(D), 1 / 6}\left(\tau / D^{2}\right)}-\omega^{2} \overline{R^{(D), 1 / 2}\left(\tau / D^{2}\right)} / \sqrt{-3} .
$$


Rewriting the RHS using Lemma 28, then

$$
\chi_{\pi}(3) R_{D, 1 / 6}\left(\tau_{\mathscr{A}^{\prime}}\right)=\frac{G\left(\chi_{\pi}\right)}{\pi}\left(\overline{R_{D, 1 / 6}(\tau)}-\omega^{2} \overline{R_{D, 1 / 2}(\tau)} / \sqrt{-3}\right) .
$$

Finally, since we noted that $R_{D, 1 / 2}(z)=0$ in the proof of Lemma 23, for any $z$, then we get $R_{D, 1 / 6}\left(\tau_{\mathscr{A}^{\prime}}\right)=\chi_{\bar{\pi}}(3) \frac{G\left(\chi_{\pi}\right)}{\pi} \overline{R_{D, 1 / 6}(\tau)}$.

Now using the details of the proof of Lemma 26, recall that

$$
R_{D, 1 / 6}\left(\tau_{\mathscr{A}^{\prime}}\right)=R_{D, 1 / 6}\left(\tau_{\mathscr{A}} / 3\right)=\chi_{\bar{\pi}}\left(n_{a}\right) R_{D, 1 / 6}(\tau / 3)=\chi_{\bar{\pi}}\left(n_{a^{\prime}}\right) R_{D, 1 / 6}(\tau)
$$

thus

$$
R_{D, 1 / 6}(\tau)=\chi_{\pi}\left(n_{a^{\prime}}\right) \chi_{\bar{\pi}}(3) \frac{G\left(\chi_{\pi}\right)}{\pi} \overline{R_{D, 1 / 6}(\tau)}
$$

To actually compute the term on the RHS, we recall a few facts about cubic Gauss sums (see [13, Chapter 9]). We can write $\pi=\prod_{p_{i} \mid D} \pi_{i}$, where $\pi_{i}$ is a generator of norm $p_{i}$ with $\pi_{i} \equiv 1(3)$. Then:

$$
G\left(\chi_{\pi}\right)=\prod_{p_{i} \mid D} \chi_{\pi / \pi_{i}}\left(\pi_{i}\right) G\left(\chi_{\pi_{i}}\right) .
$$

Moreover, we can actually compute each $G\left(\chi_{\pi_{i}}\right)$ up to a cubic root of unity. From [13, Chapter 9.4], we have $G\left(\chi_{\pi_{i}}\right)^{3}=-p \bar{\pi}_{i}$ for $\pi_{i} \equiv 1 \bmod 3$. Thus we get $G\left(\chi_{\pi_{i}}\right)=-\bar{\pi}_{i}^{2 / 3} \pi_{i}^{1 / 3} \omega^{k_{i}}$ for some $k_{i} \in\{0,1,2\}$,

Then $\frac{G\left(\chi_{\pi}\right)}{\pi}=(-1)^{\sigma(D)} \omega^{k_{D}} \frac{\bar{\pi}^{2 / 3}}{\pi^{2 / 3}}$, where $k_{D}=\sum k_{i}$, which together with (24) gives us Proposition 27 for $\omega^{k^{\prime}}=\chi_{\pi}\left(n_{a^{\prime}}\right) \chi_{\bar{\pi}}(3) \omega^{k_{D}}$.

From Lemma 26 and Proposition 27, we get $T_{D}=R_{D, 1 / 6}(\tau / 3) \bar{\pi}^{-2 / 3} \omega^{k_{0}}$ for $k_{0}=k+k^{\prime}$, and thus:

Corollary $29 S_{D}=(-1)^{\sigma(D)} T_{D}^{2}$.

\subsection{Invariance under the Galois action}

Define

$$
M_{D}=R_{D, 1 / 6}(\tau / 3) \bar{\pi}^{-2 / 3}
$$

We will write below $M_{D}$ as a trace.

Lemma $30 M_{D} \in K$ and we can write it as a trace:

$$
M_{D}=\frac{1}{\varphi(D)} \operatorname{Tr}_{H_{\mathscr{O}} / K}\left(f_{1,1 / 6}(\tau) \bar{\pi}^{-2 / 3}\right)
$$


where $H_{\mathscr{O}}$ is the ray class field of modulus $3 D, \varphi$ is Euler's totient function and $f_{1,1 / 6}(\tau)=\theta_{1,1 / 6}(\tau) / \theta_{0}(\tau)$

Recall we defined $T_{D}=R_{D, 1 / 6}(\tau) \bar{\pi}^{-2 / 3} \omega^{k^{\prime}}$ and from Lemma 26 we have $T_{D}=$ $R_{D, 1 / 6}(\tau / 3) \bar{\pi}^{-2 / 3} \omega^{k_{0}}$ for $k_{0}=k+k^{\prime}$. As $T_{D}=(-1)^{\sigma} \overline{T_{D}}$ from Proposition 27, we get immediately from Lemma 30 :

Corollary $31 T_{D} \in \mathbb{Q}$ when $\sigma(D)$ even and $T_{D} / \sqrt{-3} \in \mathbb{Q}$ when $\sigma(D)$ odd and we have the formula:

$$
T_{D}=\frac{\omega^{k_{0}}}{\varphi(D)} \operatorname{Tr}_{H_{\mathscr{O}} / K}\left(f_{1,1 / 6}(\tau) \bar{\pi}^{-2 / 3}\right)
$$

Proof of Lemma 30: We can write explicitly $M_{D}=\sum_{r \in \underset{r \equiv 1(6)}{(\mathbb{Z} / D \mathbb{Z}} \times} f_{r, 1 / 6}(\tau) \chi_{\pi}(r) \bar{\pi}^{-2 / 3}$. For an ideal $\mathscr{A}=\left[a, \frac{-b+\sqrt{-3}}{2}\right]_{\mathbb{Z}}$ with generator $k_{a}=\left(n_{a} a+m_{a} \frac{-b+\sqrt{-3}}{2}\right)$ with $6 \mid m_{a}, n_{a} \equiv 1(6)$, we are going to have the Galois transformation:

$$
\left(f_{r, 1 / 6}(\tau) \chi_{\pi}(r) \bar{\pi}^{-2 / 3}\right)^{\sigma_{\mathscr{A}}^{-1}}=f_{n_{a} r, 1 / 6}(\tau) \chi_{\pi}\left(n_{a} r\right) \bar{\pi}^{-2 / 3} .
$$

To show this, note that from Lemma 20 we have $f_{r, 1 / 6}(\tau)^{\sigma_{\mathscr{A}}^{-1}}=f_{r n_{a}, 1 / 6}(\tau)$. We compute $\left(\bar{\pi}^{1 / 3}\right)_{\mathscr{A}}^{\sigma^{-1}}=\chi_{\bar{\pi}}(\mathscr{A}) \bar{\pi}^{1 / 3}$. Furthermore, $\chi_{\bar{\pi}}(\mathscr{A})=\overline{\left(\frac{\bar{\pi}}{\mathscr{A}}\right)_{3}}=\left(\frac{\pi}{\mathscr{A}}\right)_{3}=$ $\left(\frac{\pi}{n_{a} a+m_{a} b}\right)_{3}$ and $n_{a}\left(n_{a} a+m_{a} b\right)^{2} \equiv a \bmod \pi$, so we have $\left(\frac{\pi}{n_{a} a+m_{a} b}\right)_{3}=\left(\frac{\pi}{n_{a}^{-1}}\right)_{3}=$ $\overline{\left(\frac{\pi}{n_{a}}\right)_{3}}=\chi_{\pi}\left(n_{a}\right)$.

Moreover, taking the ideals $\mathscr{A}_{r}^{\circ}=\left(1+b^{*}\left(1-r^{*}\right) \frac{-b+\sqrt{-3}}{2}\right)$, where $b^{*} \equiv b^{-1}$ $\bmod D$ and $r^{*} \equiv r^{-1} \bmod D$, we have $\operatorname{Nm} \mathscr{A}_{r}^{\circ}=a_{\mathscr{A}_{r}^{\circ}} \equiv r^{-1} \bmod 3 D$ and $n_{\mathscr{A}_{r}^{\circ}} \equiv$ $r \bmod 3 D$, and then:

$$
M_{D}=\sum_{\substack{r \in(\mathbb{Z} / D \mathbb{Z})^{\times} \\ r \equiv 1(6)}}\left(f_{1,1 / 6}(\tau) \bar{\pi}^{-2 / 3}\right)_{\mathscr{A}_{r}^{\circ}}^{\sigma^{-1}}
$$

We will show in the next section in Proposition 40 that $f_{1,1 / 6}(\tau) \in H_{\mathscr{O}}$. Define the group $G_{0}=\left\{\left[\mathscr{A}_{r}^{\circ}\right], r \in(\mathbb{Z} / D \mathbb{Z})^{\times}, r \equiv 1 \bmod 6\right\}$. It is a subgroup of $\operatorname{Gal}\left(H_{\mathscr{O}} / K\right)$ and $G_{0}$ is isomorphic to $(\mathbb{Z} / D \mathbb{Z})^{\times}$. We define the fixed field of $G_{0}$ in $\operatorname{Gal}\left(H_{\mathscr{O}} / K\right)$ to be $H_{0}=\left\{h \in H_{\mathscr{O}}: \sigma(h)=h, \forall \sigma \in G_{0}\right\}$ and Galois theory implies $\operatorname{Gal}\left(H_{\mathscr{O}} / H_{0}\right) \cong G_{0}$. Then we can rewrite the relation above as

$$
M_{D}=\operatorname{Tr}_{H_{\mathscr{O}} / H_{0}}\left(f_{1,1 / 6}(\tau) \bar{\pi}^{-2 / 3}\right)
$$

As we will show in the next section in Proposition 40, $f_{r, 1 / 6}(\tau)$ with $r \equiv 1 \bmod 6$ are all the Galois conjugates of $f_{1,1 / 6}(\tau)$, thus $M_{D} \in K$. Then we can take the trace 
further to $K$ to get

$$
\operatorname{Tr}_{H_{\mathscr{O}} / K}\left(f_{1}(\tau) \bar{\pi}^{-2 / 3}\right)=\# \operatorname{Gal}\left(H_{0} / K\right) \operatorname{Tr}_{H_{\mathscr{O}} / H_{0}}\left(f_{1,1 / 6}(\tau) \bar{\pi}^{-2 / 3}\right)
$$

and this equals $\prod_{p \mid D}(p-1) \operatorname{Tr}_{H_{\mathscr{O}} / H_{0}}\left(f_{1,1 / 6}(\tau) \bar{\pi}^{-2 / 3}\right)$.

Remark 32 Using the notation from the proof of Lemma 30, one can actually show similarly that $\kappa=R_{D, 1 / 6} D^{-1 / 3}$ equals:

$$
\kappa=\operatorname{Tr}_{H_{\mathscr{O}} / H_{0}}\left(f_{1}(\tau) D^{-1 / 3}\right)
$$

and $\kappa^{3} \in K$. However, $\kappa \notin K$.

\subsection{Integrality}

In Sect. 3 we have showed that $S_{D} \in \mathbb{Q}$. We will show below that $S_{1 / 6} \in \mathbb{Z}$, thus $3 c_{3 D} S_{D} \in \mathbb{Z}$.

Recall that $S_{1 / 6}=3 c_{3 D} S_{D}=\operatorname{Tr}_{H_{3 D} / K} \frac{\Theta(D \omega)}{\Theta(\omega)} D^{1 / 3}$. Note that it is enough to show that $D^{1 / 3} \Theta(D \omega) / \Theta(\omega)$ is an algebraic integer, as its trace would be a rational number as well as an algebraic integer, thus an integer. Moreover, it is enough to show that $\Theta(D \omega) / \Theta(\omega)$ is an algebraic integer.

We cite the following standard result (see for example [19]):

Lemma 33 Let $f(z)$ be a modular function for $\Gamma(N)$ such that for all $\gamma \in \mathrm{SL}_{2}(\mathbb{Z})$ we have $f \circ \gamma$ holomorphic on the upper half plane $\mathscr{H}$ and $f$ has Fourier coefficients at $\infty$ that are algebraic integers. Then, for $\tau$ a CM point, $f(\tau)$ is an algebraic integer.

First we will show that $2 \frac{\Theta(D \omega)}{\Theta(\omega)}$ is an algebraic integer. We have showed that $\frac{3}{2} \Theta(\omega)=\sqrt[4]{3}\left|\theta_{0}(\tau)\right|^{2}$. Then $2 \frac{\Theta(D \omega)}{\Theta(\omega)}=3^{3 / 4} \frac{\Theta(D \omega)}{\left|\theta_{0}(\tau)\right|^{2}}=3^{3 / 4} e^{-2 \pi i / 24} \frac{\Theta(-D \bar{\tau})}{\theta_{0}(-\bar{\tau})^{2}}$.

Since $e^{-2 \pi i / 24} 3^{3 / 4}$ is an algebraic integer, it is enough to show that $\frac{\Theta(-D \bar{\tau})}{\theta_{0}(-\bar{\tau})^{2}}$ is one as well. Recall that $\theta_{0}(z)=\eta(z / 3)$ and take $f_{0}(z)=\frac{\Theta(D z)}{\eta(z / 3)^{2}}$.

Note that:

- $f_{0}$ is a modular function for $\Gamma(36 D)$;

- $f_{0}(\gamma z)$ is holomorphic on $\mathscr{H}$ for all $\gamma \in \mathrm{SL}_{2}(\mathbb{Z})$;

- $f_{0}(\gamma z)$ has Fourier coefficients that are algebraic integers in its Fourier expansion at $\infty$ for all $\gamma \in \mathrm{SL}_{2}(\mathbb{Z})$.

These properties can be checked using the properties of $\Theta_{K}$ from the Appendix $((27)$ and Lemma 41), as well as the properties of $\eta(z)$. Note that we are in the conditions of Lemma 33, thus $f(\tau)$ is an algebraic integer. This implies that $2 \frac{\Theta(D \omega)}{\Theta(\omega)}$ is an algebraic integer, hence $2 S_{1 / 6}$ is an integer.

Now we will show that $D S_{1 / 6}$ is an integer as well by showing that $D \frac{2}{3} \frac{\Theta_{1 / 6}(D \omega)}{\Theta(\omega)}$ is an algebraic integer. Using Lemma 16 for $\mu \in\{-1 / 2,-1 / 6\}, v=1 / 2, a=1$, 
$z=D \frac{-3+\sqrt{-3}}{2}$, we can rewrite:

$$
\Theta_{1 / 6}(D \tau)=\frac{\sqrt[4]{3}}{D} \sum_{r=0}^{D-1}\left|\theta_{r, 1 / 6}(\tau)\right|^{2}, \tau=\frac{-3+\sqrt{-3}}{2} .
$$

Taking the quotient by $\frac{3}{2} \Theta(\tau)=\sqrt[4]{3}\left|\theta_{0}(\tau)\right|^{2}$, we get:

$$
\frac{2}{3} \frac{\Theta_{1 / 6}(D \omega)}{\Theta(\omega)}=\frac{1}{D} \sum_{r=0}^{D-1}\left|\frac{\theta_{r, 1 / 6}(\tau)}{\theta_{0}(\tau)}\right|^{2}
$$

Recall $f_{r, \mu}(z)=\frac{\theta_{r, \mu}(z)}{\theta_{0}(z)}$ for $\mu \in\{1 / 2,1 / 6\}$ and note that:

- $f_{r, \mu}$ is a modular function for $\Gamma\left(18 D^{2}\right)$;

- $f_{r, \mu}(\gamma z)$ is holomorphic on $\mathscr{H}$ for all $\gamma \in \mathrm{SL}_{2}(\mathbb{Z})$;

- $f_{r, \mu}(\gamma z)$ has Fourier coefficients that are algebraic integers in its Fourier expansion at $\infty$ for all $\gamma \in \mathrm{SL}_{2}(\mathbb{Z})$.

To see the last property, we can either compute explicitly the transformations of $\theta_{r, \mu}(\gamma z)$ and $\theta_{0}(\gamma z)$ and get a Fourier expansion with coefficients in $\mathscr{O}_{K}\left[\zeta_{24}, \zeta_{D^{2}}\right]$, or interpret the theta functions as generating series attached to counting points on certain lattices and get the Fourier coefficients of their ratio to be in $\overline{\mathbb{Z}}$.

Thus we are in the conditions of Lemma 33, hence $f_{r, \mu}(\tau)$ is an algebraic integer. This implies that $D \frac{\Theta_{1 / 6}(D \omega)}{\Theta(\omega)}$ is an algebraic integer, and thus the trace $D S_{1 / 6}=$ $D \operatorname{Tr}_{H_{3 D} / K} \frac{\Theta_{1 / 6}(D \omega)}{\Theta(\omega)} D^{1 / 3}$, which is a rational number, is indeed an integer. Since we already showed that $2 S_{1 / 6}$ is an integer, we get $S_{1 / 6} \in \mathbb{Z}$ when $D$ is odd.

If we note that $\left|\theta_{0}(\tau)\right|^{2}=|\eta(\tau / 3)|^{2}=\sqrt{3}|\eta(\tau)|^{2}$, then we can also show similarly that $\frac{\theta_{r, \mu}(\tau)}{\eta(\tau)}$ is an algebraic integer, and thus $D S_{1 / 6} / \sqrt{3}$ is an algebraic integer. Since $S_{1 / 6} \in \mathbb{Z}$, this implies 3 divides $S_{1 / 6}$.

Finally, since $S_{1 / 6}=(-1)^{\sigma_{D}} T_{D}^{2}$ and $S_{1 / 6}$ is an integer, from Corollary 31 we get:

Corollary $34 T_{D} / 3 \in \mathbb{Z}$ when $\sigma(D)$ even and $T_{D} / \sqrt{-3} \in \mathbb{Z}$ when $\sigma(D)$ odd.

Note that this implies that $\omega^{k_{0}}$ is the unique choice for a cube root of unity such that $T_{D}$ or $T_{D} / \sqrt{-3}$ is an integer.

\subsection{Case of $D$ not square free}

We present below the case of $D$ not square free. We write $D=D_{1} D_{2}^{2}$ such that $D_{1} D_{2}$ square free. Note that if we try to apply the steps before directly to (9) for $D$ not square-free some of the details of Proposition 22 no longer hold. Instead we use the formula (13):

$$
S_{D}=\frac{1}{3} \sum_{\mathscr{A} \in \operatorname{Cl}\left(\mathscr{O}_{3 D_{0}}\right)} \frac{\Theta_{K}\left(D_{0} \tau_{\mathscr{A}}\right)}{\Theta_{K}\left(\tau_{\mathscr{A}}\right)} \chi_{D}(\mathscr{A}) D^{1 / 3} .
$$


for $D_{0}=D_{1} D_{2}$. All the details of the proof for the square-free case will follow through and we only briefly mention the steps.

We apply the factorization formula and obtain the factorization from Corollary 19:

$$
\frac{\Theta_{\mu}\left(D_{0} \tau_{\mathscr{A}}\right)}{\Theta\left(\tau_{\mathscr{A}}\right)}=\frac{3 / 2}{D_{0}} \sum_{r \in \mathbb{Z} / D_{0} \mathbb{Z}} f_{a r, \mu}\left(\tau_{\mathscr{A}^{2} \mathscr{A}_{1}}\right) \overline{f_{r, \mu}\left(\tau_{\mathscr{A}_{1}}\right)}
$$

We use this to show similarly to the proof of Proposition 24 that:

$$
S_{D}=\frac{D_{2}^{1 / 3} D_{1}^{-2 / 3}}{3 c_{3 D}}\left|\sum_{\substack{s \in\left(\mathbb{Z} / D_{0} \mathbb{Z}\right)^{\times} \\ s \equiv 1(6)}} f_{s, 1 / 6}(\tau) \chi_{D}\left(\mathscr{A}_{s}\right)\right|^{2},
$$

where $\mathscr{A}_{s}=\left[a_{s}, \frac{-b+\sqrt{-3}}{2}\right]$ with $\operatorname{Nm}\left(a_{s}\right) \equiv s \bmod D_{1} D_{2}$. Take $\pi_{1}$ a generator of $D_{1}$ and $\pi_{2}$ is the generator of $D_{2}$ such that $\left(\frac{-b+\sqrt{-3}}{2}\right)$ is divisible by $\left(\pi_{1} \pi_{2}\right)^{2}$. Then $\chi_{D}\left(\mathscr{A}_{S}\right)=\chi_{\pi_{1}}(s) \overline{\chi_{\pi_{2}}(s)}$.

The main difference is when we compute the complex conjugate of

$$
R_{D, 1 / 6}(\tau)=\sum_{\substack{r \in\left(\mathbb{Z} / D_{0} \mathbb{Z}\right)^{\times} \\ r \equiv 1(6)}} f_{r, 1 / 6}(\tau) \chi_{\pi_{1}}(s) \overline{\chi_{\pi_{2}}(s)},
$$

as we get $R_{D, 1 / 6}(\tau)=\frac{G\left(\chi_{\pi_{1}} \chi_{\bar{\pi}}\right)}{\pi_{1} \pi_{2}} \overline{R_{D, 1 / 6}(\tau)}$ and this equals

$$
R_{D, 1 / 6}(\tau)=(-1)^{\sigma(D)} \omega^{k^{\prime}} \frac{\bar{\pi}_{1}^{1 / 3} \bar{\pi}_{2}^{2 / 3}}{\pi_{1}^{1 / 3} \pi_{2}^{1 / 3}} \overline{R_{D, 1 / 6}(\tau)}
$$

for a cubic root of unity $\omega^{k^{\prime}}$. Then we can rewrite:

$$
S_{D}=\frac{(-1)^{\sigma(D)}}{3 c_{3 D}} T_{D}^{2}
$$

where $T_{D}=\sum_{\substack{s \in\left(\mathbb{Z} / D_{1} D_{2} \mathbb{Z}\right)^{\times} \\ s \equiv 1(6)}} f_{s, 1 / 6}(\tau) \chi_{\pi_{1}}(s) \overline{\chi_{\pi_{2}}(s)} \bar{\pi}_{1}-2 / 3 \pi_{2}^{1 / 3} \omega^{k_{0}}$, and we can show that this is the trace:

$$
T_{D}=\frac{1}{\varphi\left(D_{0}\right)} \operatorname{Tr}_{H_{\mathscr{O}} / K} f_{1}(\tau) \bar{\pi}_{1}-2 / 3 \pi_{2}^{1 / 3} \omega^{k_{0}}
$$

where $H_{\mathscr{O}}$ is the ray class field for the modulus $D_{0}$ and $\omega^{k_{0}}$ is a cubic root of unity. Moreover, we can further show as in Sect. 4.5 that $T_{D} / 3 \in \mathbb{Z}$ when $\sigma(D)$ even and $T_{D} / \sqrt{-3} \in \mathbb{Z}$ when $\sigma(D)$ odd.

Finally, we have $S_{D} \neq 0$ for $D$ split only for $D \equiv 1 \bmod 9$ and in this case we can compute the Tamagawa number $c_{3 D}$ to be equal to $3^{1+\sigma(D)}$ using Tate's algorithm 
(see [28, Chapter IV.9]). Thus $S_{D}$ is an integer square up to an even power of 3 and it equals:

$$
S_{D}=\left(\frac{T_{D}}{(\sqrt{-3})^{2+\sigma(D)}}\right)^{2}
$$

\section{Shimura reciprocity law}

We present below some background on Shimura's reciprocity law following the exposition of Stevenhagen [30]. For more details also see Gee [7].

Let $\mathscr{F}$ be the field of modular functions over $\mathbb{Q}$. From CM theory (see for example [30]), it is known that if $\tau \in K \cap \mathscr{H}$ and $f \in \mathscr{F}$, then we have $f(\tau) \in K^{a b}$, where $K^{a b}$ is the maximal abelian extension of $K$. Shimura's reciprocity law gives us a way to compute the Galois conjugates $f(\tau)^{\sigma}$ of $f(\tau)$ when acting with $\sigma \in \operatorname{Gal}\left(K^{a b} / K\right)$. We recall that $\mathscr{F}=\bigcup_{N \geq 1} \mathscr{F}_{N}$, where $\mathscr{F}_{N}$ is the space of modular functions of level $N$.

As the restriction maps between the fields $\mathscr{F}_{N}$ are in correspondence with the natural maps between the groups $\mathrm{GL}_{2}(\mathbb{Z} / N \mathbb{Z}) /\{ \pm 1\}$, we can take the projective limit to get the isomorphism $\operatorname{Gal}\left(\mathscr{F} / \mathscr{F}_{1}\right) \cong \mathrm{GL}_{2}(\widehat{\mathbb{Z}}) /\{ \pm 1\}$.

When working over $\mathbb{Q}$, one has an isomorphism $\operatorname{Gal}\left(\mathscr{F}_{N} / \mathscr{F}_{1}\right) \cong \mathrm{GL}_{2}(\mathbb{Z} / N \mathbb{Z}) /\{ \pm 1\}$ and the maps on $\mathscr{F}_{N}$ are given by projecting $\mathrm{GL}_{2}(\widehat{\mathbb{Z}}) /\{ \pm 1\} \rightarrow \mathrm{GL}_{2}(\mathbb{Z} / N \mathbb{Z}) /\{ \pm 1\}$. We define the actions of $\mathrm{GL}_{2}(\mathbb{Z} / N \mathbb{Z}) /\{ \pm 1\}$ explicitly:

- Action of $\alpha \in \mathrm{SL}_{2}(\mathbb{Z} / N \mathbb{Z})$ on $\mathscr{F}_{N}$. We have $(f(\tau))^{\sigma_{\alpha}}=f^{\alpha}(\tau)=f(\alpha \tau)$, where $\alpha$ is acting on the upper half plane via fractional linear transformations.

- Action of $\left(\begin{array}{ll}1 & 0 \\ 0 & d\end{array}\right) \in(\mathbb{Z} / N \mathbb{Z})^{\times}$on $\mathscr{F}_{N}$. Note that for $f \in \mathscr{F}_{N}$ we have a Fourier expansion $f(z)=\sum_{n \geq 0} a_{n} q^{n / N}$ with coefficients $a_{n} \in \mathbb{Q}\left(\zeta_{N}\right), q=e^{2 \pi i z}$. If we denote $u_{d}=\left(\begin{array}{ll}1 & 0 \\ 0 & d\end{array}\right)$, then the action of $\sigma_{u_{d}}$ is given by $(f(\tau))^{\sigma_{u_{d}}}=f^{u_{d}}(\tau)=$ $\sum_{n \geq 0} a_{n}^{\sigma_{d}} q^{n / N}$,

where $\sigma_{d}$ is the Galois action in $\operatorname{Gal}\left(\mathbb{Q}\left(\zeta_{N}\right) / \mathbb{Q}\right)$ that sends $\zeta_{N} \rightarrow \zeta_{N}^{d}$.

To further get all the automorphisms of $\mathscr{F}$ we need to consider the action of $\mathrm{GL}_{2}\left(\mathbb{A}_{\mathbb{Q}, f}\right)$ and for that it is enough to define the action of $\mathrm{GL}_{2}(\mathbb{Q})^{+}$on $\mathscr{F}$ :

- Action of $\alpha \in \mathrm{GL}_{2}(\mathbb{Q})^{+}$on $\mathscr{F}$. We define $f^{\alpha}(\tau)=f(\alpha \tau)$, where $\alpha$ acts by fractional linear transformations.

Let $\mathscr{O}$ be the order of $K$ generated by $\tau$ i.e. $\mathscr{O}=\mathbb{Z}[\tau]$. We define the matrix $g_{\tau}(x)$ to be the unique matrix in $\mathrm{GL}_{2}\left(\mathbb{A}_{\mathbb{Q}}\right)$ such that $x\left(\begin{array}{l}\tau \\ 1\end{array}\right)=g_{\tau}(x)\left(\begin{array}{l}\tau \\ 1\end{array}\right)$. We can compute it explicitly. To do that, consider the minimal polynomial of $\tau$ to be $p(X)=X^{2}+B X+C$. Then if we write $x_{p} \in \mathbb{Q}_{p}^{\times}$in the form $x_{p}=s_{p} \tau+t_{p} \in \mathbb{Q}_{p}^{\times}$with $s_{p}, t_{p} \in \mathbb{Q}_{p}$, we can compute $g_{\tau}\left(x_{p}\right)=\left(\begin{array}{cc}t_{p}-s_{p} B & -s_{p} C \\ s_{p} & t_{p}\end{array}\right)$.

Using the map $g_{\tau}$ above, we have: 
Theorem 35 (Shimura's reciprocity law) For $f \in \mathscr{F}$ and $x \in \mathbb{A}_{K, f}^{\times}$, we have:

$$
(f(\tau))^{\sigma_{x}}=f^{g_{\tau}\left(x^{-1}\right)}(\tau),
$$

where $\sigma_{x}$ is the Galois action corresponding to the idele $x$ via the Artin map, $g_{\tau}$ is defined above and the action of $g_{\tau}(x)$ is the action in $\mathrm{GL}_{2}\left(\mathbb{A}_{\mathbb{Q}, f}\right)$.

Note that the elements of $K^{\times}$have trivial action. This can be easily seen by embed$\operatorname{ding} K^{\times} \hookrightarrow \mathrm{GL}_{2}(\mathbb{Q})^{+}$via $k \hookrightarrow g_{\tau}(k)$. Noting that $\tau$ is fixed by the action of the torus $K^{\times}$, we have $f^{g_{\tau}\left(k^{-1}\right)}(\tau)=f\left(g_{\tau}\left(k^{-1}\right) \tau\right)=f(\tau)$.

We can also rewrite the theorem for ideals in $K$. Let $f \in \mathscr{F}_{N}$ and $\mathscr{O}=\mathbb{Z}[\tau]$ of conductor $M$. Going through the Artin map, we can restate Shimura's reciprocity in this case in the form:

$$
f(\tau)^{\sigma_{\mathscr{A}}}=f^{g_{\tau}(\mathscr{A})^{-1}}(\tau)
$$

where $\mathscr{A}$ is an ideal prime to $M N, \sigma_{\mathscr{A}}$ is the Galois action corresponding to the ideal $\mathscr{A}$ through the Artin map, and $g_{\tau}(\mathscr{A})=g_{\tau}\left((\alpha)_{p \mid \operatorname{Nm}(\mathscr{A})}\right)$. Note that $g_{\tau}(\mathscr{A})$ is unique up to multiplication by roots of unity in $K$. However, these have trivial action on $f$ at the unramified places $p \mid \mathrm{Nm}(\mathscr{A})$.

\subsection{Galois conjugates of $f(\omega)$}

We denote $f(z)=\frac{\Theta_{K}(D z)}{\Theta_{K}(z)}$. We are interested in finding the Galois conjugates of $f(\omega)$. By checking directly computationally and using the fact that both $\Theta_{K}(D z)$ and $\Theta_{K}(z)$ have Fourier expansions in $(\mathbb{Z} \llbracket q \rrbracket)^{\times}$, where $q=e^{2 \pi i z}$, we get the standard result:

Lemma 36 The function $f(z)$ is a modular function of level $3 D$ with integer Fourier coefficients at the cusp $\infty$.

From CM-theory, if $f \in \mathscr{F}_{3 D}$ and $\tau$ a generator of $\mathscr{O}_{K}$, we have $f(\tau) \in H_{\mathscr{O}}$ the ray class field of modulus $3 D$. Recall $H_{3 D}$ is the ring class field for the order $\mathscr{O}_{3 D}=$ $\mathbb{Z}+3 D \mathscr{O}_{K}$, and we actually have:

Lemma $37 f(\omega) \in H_{3 D}$.

We give below a computational proof. However this can also be seen via Katz's modular interpretation of algebraic modular forms by thinking of $\omega \in \mathscr{H}$ corresponding to a point $[(E, C)] \in Y_{0}(3 D)\left[H_{3 D}\right]$ (where $E$ is an elliptic curve and $C$ is a torsion subgroup of $E(\mathbb{C})$ of degree $3 D)$, via the projection map $\mathscr{H} \rightarrow Y_{0}(3 D)=\mathscr{H} / \Gamma_{0}(3 D)$ (see Katz [15] for more details).

Proof We need to show that $f(\omega)$ is invariant under $\operatorname{Gal}\left(K^{a b} / H_{3 D}\right)$. Via the Artin map, we can pick as representatives the ideal classes $\mathscr{A}=\left[a, \frac{-b+\sqrt{-3}}{2}\right]_{\mathbb{Z}}$, where $a=\mathrm{Nm} \mathscr{A}$ and $b^{2} \equiv-3 \bmod 4 a$, corresponding to the Galois classes of $\operatorname{Gal}\left(K^{a b} / H_{3 D}\right)$. Then we can pick a generator of the ideal $\mathscr{A}$ written in the form $t a+s \frac{-b+\sqrt{-3}}{2}$ where $t, s \in \mathbb{Z}$ 
and $3 D \mid s$. As $f(\omega)=f(\tau)$, where $\tau=\frac{-b+\sqrt{-3}}{2}$, from Shimura's reciprocity law, we have:

$$
(f(\tau))^{\sigma_{\mathscr{A}}-1}=f^{g_{\tau}(\mathscr{A})}(\tau) .
$$

Here $g_{\tau}(\mathscr{A})=\left(\begin{array}{cc}t a-s b & -s c a \\ s & t a\end{array}\right)_{p \mid 3 D}$, where $c a=\frac{b^{2}+3}{4}$. Then we can rewrite the action of $g_{\tau}(\mathscr{A})$ explicitly as:

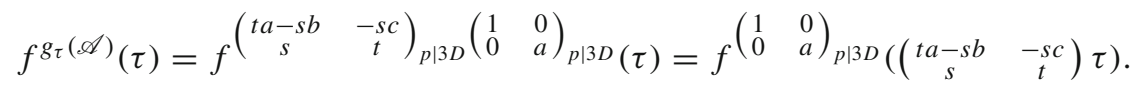

Since $3 D \mid s$, the matrix $\left(\begin{array}{cc}t a-s b & -s c \\ s & t\end{array}\right) \in \Gamma_{0}(3 D)$ and $f(z)$ is invariant under its action, while, since $(a, 3 D)=1$ and $f$ has rational Fourier coefficients at $\infty$, the action of $\left(\begin{array}{ll}1 & 0 \\ 0 & a\end{array}\right)_{p \mid 3 D}$ is trivial. Thus $f(\omega)$ is invariant under the Galois action coming from $U(3 D)$ and this finishes the proof.

Now we would like to compute the Galois conjugates of $f(\omega)$ under the action of $\operatorname{Gal}\left(H_{3 D} / K\right)$. We will first show the following general result:

Lemma 38 Let $F \in \mathscr{F}_{N}$ be a modular function of level $N$ with rational Fourier coefficients in its Fourier expansion at $\infty$. Let $\tau=\frac{-b+\sqrt{-3}}{2}$ be a CM point and let $\mathscr{A}=\left[a, \frac{-b+\sqrt{-3}}{2}\right]_{\mathbb{Z}}$ be a primitive ideal prime to $N$. Then we have the Galois action:

$$
F(\tau)^{\sigma_{\mathscr{A}}^{-1}}=F(\tau / a)
$$

Proof From Shimura's reciprocity law, we have $F(\tau)^{\sigma_{\mathscr{A}}^{-1}}=F^{g_{\tau}(\mathscr{A})}(\tau)$. Note that the minimal polynomial of $\tau$ is $p_{\tau}(X)=X^{2}+b X+\frac{b^{2}+3}{4}$. Let $\alpha$ be a generator of $\mathscr{A}$. Then we can write $\alpha$ in the form $\alpha=t a+s \tau$ and we have $g_{\tau}(\mathscr{A})=\left(\begin{array}{cc}t a-s b & -s \frac{b^{2}+3}{4} \\ -s & t a\end{array}\right)_{p \mid a}$. We can rewrite the matrix in the form $g_{\tau}(\mathscr{A})=\left(\begin{array}{cc}t a-s b & \frac{b^{2}+3}{4 a} \\ -s & t\end{array}\right)_{p \mid a}\left(\begin{array}{ll}1 & 0 \\ 0 & a\end{array}\right)_{p \mid a}$. As the matrix $\left(\begin{array}{cc}t a-s b & -\frac{b^{2}+3}{4 a} \\ -s & t\end{array}\right)_{p \mid a}$ is an element of $\operatorname{SL}_{2}\left(\mathbb{Z}_{p}\right)$ for $p \nmid N$, it has trivial action.

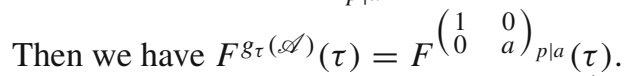

We rewrite the matrix $\left(\begin{array}{ll}1 & 0 \\ 0 & a\end{array}\right)_{p \mid a}=\left(\begin{array}{cc}1 & 0 \\ 0 & 1 / a\end{array}\right)_{p \nmid a}\left(\begin{array}{ll}1 & 0 \\ 0 & a\end{array}\right)_{\mathbb{Q}}$, where $\left(\begin{array}{cc}1 & 0 \\ 0 & 1 / a\end{array}\right)_{p \nmid a} \in$ $\mathrm{GL}_{2}(\widehat{\mathbb{Z}})$ and $\left(\begin{array}{ll}1 & 0 \\ 0 & a\end{array}\right)_{\mathbb{Q}} \in \mathrm{GL}_{2}(\mathbb{Q})^{+}$. Note that the action of $\left(\begin{array}{cc}1 & 0 \\ 0 & 1 / a\end{array}\right)_{p \nmid a}$ is the same as the action of $\left(\begin{array}{cc}1 & 0 \\ 0 & 1 / a\end{array}\right)_{p \mid N}$. However, since $F$ has rational Fourier coefficients in its Fourier expansion, this action is trivial. Thus we are left with $F^{g_{\tau}(\mathscr{A})}(\tau)=$

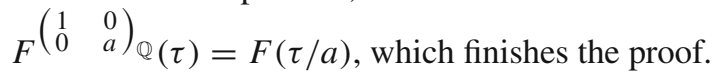

We apply the lemma above to our case: 
Proposition 39 Take the primitive ideals $\mathscr{A}=\left[a, \frac{-b+\sqrt{-3}}{2}\right]_{\mathbb{Z}}$ to be the representatives of the classes of the ring class group $\mathrm{Cl}\left(\mathscr{O}_{3 D}\right)$ such that all norms a $=\mathrm{Nm} \mathscr{A}$ are relatively prime to each other and $b^{2} \equiv-3 \bmod 4 a$ for all the norms $a$.

Then the Galois conjugates of $f(\omega)=\frac{\Theta_{K}(D \omega)}{\Theta_{K}(\omega)}$ are the terms $\left(\frac{\Theta_{K}(D \omega)}{\Theta_{K}(\omega)}\right)^{\sigma_{\mathscr{A}}^{-1}}=$ $\frac{\Theta_{K}\left(D \tau_{\mathscr{A}}\right)}{\Theta_{K}\left(\tau_{\mathscr{A}}\right)}$.

Proof We note that $\frac{\Theta_{K}(D \omega)}{\Theta_{K}(\omega)}=\frac{\Theta_{K}(D \tau)}{\Theta_{K}(\tau)}$ and apply Lemma 38 to $\tau=\frac{-b+\sqrt{-3}}{2}$ and $f(z)=\frac{\Theta_{K}(D z)}{\Theta_{K}(z)}$. These are the only Galois conjugates, as we showed that $f(\tau) \in H_{3 D}$.

\subsection{Galois conjugates of $f_{r, \mu}(\tau)$}

Recall that we defined $f_{r, \mu}(z)=\frac{\theta_{r, \mu}(z)}{\theta_{0}(z)}$. This is a modular function for $\Gamma\left(18 D^{2}\right)$ when $\mu=1 / 2$ and for $\Gamma\left(9 D^{2}\right)$ when $\mu=1 / 6$ (see Lemma 46 in the Appendix). From CM-theory then $f_{r, \mu}(\tau) \in H_{\mathscr{O}^{\prime}}$, where $H_{\mathscr{O}^{\prime}}$ is the ray class field of modulus $18 D^{2}$ for $\mu=1 / 2$ and of modulus $9 D^{2}$ for $\mu=1 / 6$. Note that this also follows from the moduli interpretation of algebraic modular forms (see [15]). However, we want to descend to the ray class field $H_{\mathscr{O}}$ of modulus $3 D$.

In order to do that we compute the Galois conjugates of $f_{r, \mu}(\tau)$ over $K$ using Shimura's reciprocity law for $\tau=\frac{-b+\sqrt{-3}}{2}$, with $b^{2} \equiv-3 \bmod 12 D^{2}$. Note that $\tau$ has the minimum polynomial $X^{2}+b X+\frac{b^{2}+3}{4}$. Thus we have to compute the action of all $g_{\tau}\left(\left(x_{p}\right)_{p}\right)=\prod_{p}\left(\begin{array}{cc}t_{p}-s_{p} b & -s_{p} \frac{b^{2}+3}{4} \\ s_{p} & t_{p}\end{array}\right)_{p}$ on $f_{r, \mu}(\tau)$. We will prove that the Galois action from Shimura's reciprocity law is given by the following:

In our case we want to compute the Galois conjugates of $f_{r, \mu}(\tau)$

Proposition 40 For $\mathscr{A}=\left[a, \frac{-b+\sqrt{-3}}{2}\right]_{\mathbb{Z}}$ an ideal prime to $6 D$ such that $b^{2} \equiv-3$ $\bmod 12 D a^{2}$. Let $k_{\mathscr{A}}=n_{a} a+m_{a} \frac{-b+\sqrt{-3}}{2}$ be the generator of $\mathscr{A}$ such that $3 \mid m_{a}$ and $n_{a} \equiv 1 \bmod 3$, then for $\tau=\frac{-b+\sqrt{-3}}{2}$ we have:

$$
f_{r, \mu}(\tau)^{\sigma_{\mathscr{A}}^{-1}}=f_{n_{a}^{\prime} r, \mu}(\tau)
$$

where $n_{a} \equiv n_{a}^{\prime} \bmod 3 D$ and $n_{a^{\prime}}$ odd. Moreover, these are all the Galois conjugates of $f_{r, \mu}(\tau)$ and $f_{r, \mu}(\tau)$ is in $H_{\mathscr{O}}$ the ray class field of modulus $3 D$.

Proof We will compute the Galois conjugates of $f_{r, \mu}(\tau)$ using Shimura's reciprocity law adelically first. As the action of $K^{\times} \prod_{v \nmid 6 D} \mathscr{O}_{K_{v}}^{\times}$is trivial, we need to compute the action of $\prod_{v \mid 6 D} \mathscr{O}_{K_{v}}^{\times}$. We project the action of $\left(g_{\tau}\left(x_{v}\right)\right)_{v \mid 6 D}$ to $g_{\tau}\left(x^{\prime}\right) \in$ $\mathrm{GL}_{2}\left(\mathbb{Z} / 18 D^{2} \mathbb{Z}\right)$. From the Chinese remainder theorem, we can find $k_{0} \in K$ such that $k_{0} \equiv x_{p} \bmod 72 D^{2} \mathbb{Z}_{p}$ for all $p \mid 6 D$. Note that $k_{0}$ is independent of the choice of $\tau$ and $g_{\tau}(x) \equiv g_{\tau}\left(k_{0}\right)$ in $\mathrm{GL}_{2}\left(\mathbb{Z} / 18 D^{2} \mathbb{Z}\right)$. 
Moreover, $\left( \pm \omega^{i}\right)_{p} \hookrightarrow \mathbb{A}_{K_{f}}^{\times}$acts trivially, thus we can consider the action of $k_{0}\left( \pm \omega^{j}\right)$ instead, for $j \in\{0,1,2\}$. We pick $k_{0}=A+B \omega$ such that $v_{3}(B) \geq 1$, $A \equiv 1(3)$. By possibly changing $b$ to $b:=b+18 D^{2}$ above (this does not affect the value of $\left.f_{r, \mu}(\tau)\right)$ as noted in Remark 47 in the Appendix), we can find $b^{\prime} \equiv b$ $\bmod 18 D^{2} \mathrm{Nm} k_{0}$ such that $k_{0}=t a+s \frac{-b^{\prime}+\sqrt{-3}}{2}$ with $t a-s b^{\prime} \equiv 1 \bmod 6$. Let $\tau^{\prime}=\frac{-b^{\prime}+\sqrt{-3}}{2}$ and we have:

$$
\left(f_{r, \mu}(\tau)\right)^{\sigma_{\left(k_{0}\right)_{6 D}}}=\left(f_{r, \mu}\left(\tau^{\prime}\right)\right)^{\sigma_{\left(k_{0}\right)_{6 D}}}=f_{r, \mu}^{g_{\tau^{\prime}}\left(\left(k_{0}\right)_{6 D}\right)}\left(\tau^{\prime}\right),
$$

where $g_{\tau^{\prime}}\left(\left(k_{0}\right)_{6 D}\right)=\left(\begin{array}{cc}t a-s b^{\prime} & -s c^{\prime} a \\ s & t a\end{array}\right)$, with $c^{\prime} a=\frac{b^{\prime 2}+3}{4}$.

We will now compute $f_{r, \mu}^{g_{\tau}(x)_{p \mid 6 D}}(\tau)$. We write the matrix $g_{\tau^{\prime}}\left(\left(k_{0}\right)_{6 D}\right)$ as a product $g_{\tau^{\prime}}\left(\left(k_{0}\right)_{6 D}\right)=\left(\begin{array}{cc}t a-s b^{\prime} & -s c^{\prime} \\ s & t\end{array}\right)_{p \mid 6 D}\left(\begin{array}{ll}1 & 0 \\ 0 & a\end{array}\right)_{p \mid 6 D}$. Note that $\left(\begin{array}{ll}1 & 0 \\ 0 & a\end{array}\right)_{p \mid 6 D}$ acts trivially on $f_{r, \mu}$ as the functions $\theta_{r, \mu}(z)$ and $\theta_{0}(z)$ have rational Fourier coefficients in its Fourier expansion at $\infty$, and thus so does $f_{r, \mu}(z)$. Thus we need to compute the action

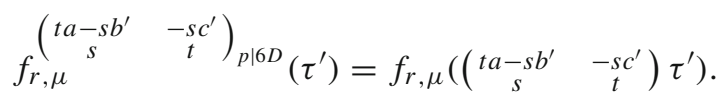

Using the transformation of Lemma 45 from the Appendix for both $\theta_{r, \mu}$ and $\theta_{0}$, as we have $3 D^{2} \mid s$ and $t a-s b \equiv 1 \bmod 6$, and further noting that $9 \mid s c^{\prime}$, we get precisely:

$$
f_{r, \mu}\left(\left(\begin{array}{cc}
t a-s b^{\prime} & -s c^{\prime} \\
s & t
\end{array}\right) \tau^{\prime}\right)=\frac{\theta_{r}\left(\left(\begin{array}{cc}
t a-s b^{\prime} & -s c^{\prime} \\
s & t
\end{array}\right) \tau^{\prime}\right)}{\theta_{0}\left(\left(\begin{array}{cc}
t a-s b^{\prime} & -s c^{\prime} \\
s & t
\end{array}\right) \tau^{\prime}\right)}=f_{\left(t a-s b^{\prime}\right) r, \mu}\left(\tau^{\prime}\right) .
$$

Since $\left(t a-s b^{\prime}\right) t \equiv 1 \bmod D^{2}$, we can rewrite this as $f_{t^{\prime-1} r, \mu}\left(\tau^{\prime}\right)$ for $t \equiv t \bmod D$ and $t^{\prime} \equiv 1 \bmod 6$. Note that $t$ is prime to $D$. Thus we have showed so far that the Galois conjugates of $f_{r, \mu}(\tau)$ are the terms $f_{s, \mu}(\tau)$, where $\operatorname{gcd}(s, D)=\operatorname{gcd}(r, D)$. Moreover, we have nontrivial Galois action only for $k_{0}=t a+s \frac{-b+\sqrt{-3}}{2}$ with $t \not \equiv 1$ mod $D$. Furthermore, it implies that $f(\tau) \in H_{\mathscr{O}}$, the ray class field of modulus $3 D$.

Finally, we would like to express the Galois action using ideals. For a primitive ideal $\mathscr{A}=\left[a, \frac{-b+\sqrt{-3}}{2}\right]_{\mathbb{Z}}$ prime to $6 D$ with a generator $\left(k_{\mathscr{A}}\right)=\left(n_{a} a+m_{a} \frac{-b+\sqrt{-3}}{2}\right)$ with $n_{a} \equiv 1 \bmod 6$ and $3 \mid m_{a}$, we have the correspondence map between ideles and ideals given by $x=\left(k_{\mathscr{A}}\right)_{p \nmid 6 D} \leftrightarrow \mathscr{A}=\left(k_{\mathscr{A}}\right)$. Picking the representatives $k_{\mathscr{A}}$ as above, we have:

$$
f_{r, \mu}(\tau)^{\sigma_{\mathscr{A}}}=f_{r, \mu}^{g_{\tau}^{-1}\left(k_{\mathscr{A}}\right)_{p \nmid 6 D}}(\tau)=f_{r, \mu}^{g_{\tau}\left(k_{\mathscr{A}}\right)_{p \mid 6 D}}(\tau)=f_{n_{a}^{\prime-1} r, \mu}(\tau),
$$

where $n_{a} \equiv n_{a}^{\prime}$ mod $3 D$ and $n_{a}^{\prime}$ odd. After changing $r \rightarrow n_{a}^{\prime-1} r$, we get the result of the Galois action from the proposition.

Acknowledgements Open access funding provided by Projekt DEAL. The author thanks Xinyi Yuan for suggesting the problem and for valuable insights. We also thank Don Zagier for encouragement to finish the result in the current form, very helpful discussions and for help with using PARI for computational 
purposes. We thank the anonymous referee for the careful feedback that helped improve the paper. We also thank the Max Planck Institute for Mathematics in Bonn and Tsinghua University in Beijing for their hospitality.

Open Access This article is licensed under a Creative Commons Attribution 4.0 International License, which permits use, sharing, adaptation, distribution and reproduction in any medium or format, as long as you give appropriate credit to the original author(s) and the source, provide a link to the Creative Commons licence, and indicate if changes were made. The images or other third party material in this article are included in the article's Creative Commons licence, unless indicated otherwise in a credit line to the material. If material is not included in the article's Creative Commons licence and your intended use is not permitted by statutory regulation or exceeds the permitted use, you will need to obtain permission directly from the copyright holder. To view a copy of this licence, visit http://creativecommons.org/licenses/by/4.0/.

\section{Appendix: Properties of theta functions}

\subsection{Properties of $\Theta_{K}$ and $\eta$}

We have a functional equation for the theta function (see [16, Section 11.1]):

$$
\Theta_{K}(-1 / 3 z)=\frac{3}{\sqrt{-3}} z \Theta_{K}(z) .
$$

Furthermore, we can compute the transformation of $\Theta_{K}(z \pm 1 / 3)$ in the lemma below:

Lemma $41 \Theta(z+k / 3)=\left(1-\omega^{2 k}\right) \Theta(3 z)+\omega^{2 k} \Theta(z)$ for $k \in \mathbb{Z}$.

Proof For $k=1$, we can split the sum $\Theta\left(z+\frac{1}{3}\right)=\sum_{m, n \in \mathbb{Z}} e^{2 \pi i\left(m^{2}+n^{2}-m n\right)\left(z+\frac{1}{3}\right)}$ in two parts, depending on whether or not the ideal $(m+n \omega)$ is prime to $(\sqrt{-3})$. The part of the sum for which $(\sqrt{-3}) \mid(m+n \omega)$ gives us $\sum_{m, n \in \mathbb{Z}} e^{2 \pi i\left(m^{2}+n^{2}-m n\right)(3 z+1)}=$ $\Theta(3 z+1)=\Theta(3 z)$.

The part of the sum for which $(\sqrt{-3}) \nmid(m+n \omega)$ can be rewritten as $\omega \sum_{\substack{m, n \in \mathbb{Z},(\sqrt{-3}) \nmid(m+n \omega)}} e^{2 \pi i\left(m^{2}+n^{2}-m n\right) z}$ since $m^{2}+n^{2}-m n \equiv 1(3)$. We rewrite this sum as the sum of two terms $\omega \sum_{m, n \in \mathbb{Z}} e^{2 \pi i\left(m^{2}+n^{2}-m n\right) z}-\omega \sum_{\substack{m, n \in \mathbb{Z},(\sqrt{-3}) \mid(m+n \omega)}} e^{2 \pi i\left(m^{2}+n^{2}-m n\right) z}$. Finally we recognize the two terms as $\omega \Theta(z)-\omega \Theta(3 z)$.

Going back to our initial computation, we get $\Theta(z+1 / 3)=\Theta(3 z)+\omega \Theta(z)-$ $\omega \Theta(3 z)=(1-\omega) \Theta(3 z)+\omega \Theta(z)$, and this finishes the proof of the first formula. We can show the case $k=2$ by applying the equality for $k=1$ and plugging in $z-1 / 3$ for $z$.

By applying the functional equation (27) for $z=\frac{-3+\sqrt{-3}}{6}$ we get the following easy lemma:

Lemma $42 \Theta_{K}\left(\frac{-3+\sqrt{-3}}{6}\right)=0$.

Using the lemma above, we get the following result that we use in the proof of Corollary 18: 
Lemma 43 For a primitive ideal $\mathscr{A}=\left[a, \frac{-b+\sqrt{-3}}{2}\right]_{\mathbb{Z}}$ prime to 3 such that $b^{2} \equiv-3$ $\bmod 12 a$, we have $\Theta_{K}\left(\frac{-b+\sqrt{-3}}{6 a}\right)=0$.

Proof The proof is similar to that of Lemma 12. We can write the generator of primitive ideal $\mathscr{A}=\left[a, \frac{-b+\sqrt{-3}}{2}\right]$ in the form $k_{\mathscr{A}}=m a+n \frac{-b+\sqrt{-3}}{2}$ for some integers $m, n$ and following similar steps as in Lemma 12 , we get $\Theta_{K}\left(\frac{-b+\sqrt{-3}}{6}\right)=(m-$ $\left.n \frac{-b+\sqrt{-3}}{2 a}\right) \Theta_{K}\left(\frac{-b+\sqrt{-3}}{6 a}\right)$. From the previous lemma, we know the LHS equals 0 , thus $\Theta_{K}\left(\frac{-b+\sqrt{-3}}{6 a}\right)=0$ as well.

We also use the following lemma in the proof of Lemma 28:

Lemma 44 For $b^{2} \equiv-3 \bmod 12 D^{2}$ and $\pi$ of norm $D$ such that the ideal $(\pi)^{2}$ divides $\left(\frac{-b+\sqrt{-3}}{2}\right)$, we have for $\tau=\frac{-b+\sqrt{-3}}{2}$ :

$$
\frac{\eta\left(\tau / D^{2}\right)}{\eta(\tau)}=(-1)^{\frac{D-1}{6}} \bar{\pi}
$$

Proof We apply Corollary 18 twice to get: $(-1)^{\frac{D-1}{6}} \frac{\theta_{0}\left(\tau / D^{2}\right)}{\theta_{0}(\tau)} \frac{\theta_{0}(\bar{\tau})}{\theta_{0}(\bar{\tau})}=\frac{\Theta_{K}(\tau / D)}{\Theta_{K}(\tau)}$.

Then from Lemma 12, we have the RHS equal to $\bar{\pi}$. Furthermore, we can pick $b \equiv b^{\prime} \bmod 8 D^{2}, b \equiv 0 \bmod 3$ and $b^{\prime} \equiv 1 \bmod 3$. Denote $\tau^{\prime}=\frac{-b^{\prime}+\sqrt{-3}}{2}$. We can pick without loss of generality $b, b^{\prime}$ such that $\left(b^{2}+3\right) / D^{2}$ and $\left(b^{\prime 2}+3\right) / D^{2}$ are prime to $D$. Then we can find ideals $\mathscr{A}, \mathscr{A}^{\prime}$ prime to $D$ such that $\mathscr{A}(\pi)^{2}(\sqrt{-3})=(\tau)$ and $\mathscr{A}^{\prime}(\pi)^{2}=\left(\tau^{\prime}\right)$. Let $a=\mathrm{Nm} \mathscr{A}, a^{\prime}=\mathrm{Nm} \mathscr{A}^{\prime}$ and then we have:

$$
\frac{\theta_{0}\left(\tau / D^{2}\right)}{\theta_{0}(\tau)}=\left(\frac{\theta_{0}\left(\tau / D^{2}\right)}{\theta_{0}(\tau)}\right)^{\sigma_{\mathscr{A}}^{-1}}=\frac{\theta_{0}\left(\frac{\tau}{a D^{2}}\right)}{\theta_{0}(\tau / a)}=\frac{\eta\left(\frac{\tau}{3 a D^{2}}\right)}{\eta\left(\frac{\tau}{3 a}\right)}=\frac{\eta(\bar{\tau})}{\eta\left(\bar{\tau} / D^{2}\right)}
$$

Similarly we compute $\frac{\eta(\bar{\tau})}{\eta\left(\bar{\tau} / D^{2}\right)}=\frac{\eta\left(\overline{\tau^{\prime}}\right)}{\eta\left(\overline{\tau^{\prime}} / D^{2}\right)}=\frac{\eta\left(\frac{\tau^{\prime}}{a^{\prime} D^{2}}\right)}{\eta\left(\frac{\tau^{\prime}}{a^{\prime}}\right)}=\left(\frac{\eta\left(\tau^{\prime} / D^{2}\right)}{\eta\left(\tau^{\prime}\right)}\right)^{\sigma_{\mathscr{A}^{\prime}}^{-1}}$. Note that we also have $\frac{\eta\left(\frac{\tau^{\prime}}{D^{2}}\right)}{\eta\left(\tau^{\prime}\right)}=\frac{\eta\left(\frac{\tau}{D^{2}}\right)}{\eta(\tau)}$, and thus we have $\frac{\eta\left(\frac{\tau}{D^{2}}\right)}{\eta(\tau)}=(-1)^{\frac{D-1}{6}} \bar{\pi}$.

\subsection{Properties of $\theta_{r, \mu}$}

Recall that for $r \in \mathbb{Z}, \mu \in\{1 / 2,1 / 6\}$, we have defined the theta function

$$
\theta_{r, \mu}(z)=\sum_{n \in \mathbb{Z}} e^{\pi i(n+r / D-\mu)^{2} z}(-1)^{n}
$$


We will write $\theta_{r, \mu}$ as an automorphic theta function (see for example [10]). Using the standard notation (see [33]):

$$
m(a)=\left(\begin{array}{cc}
a & 0 \\
0 & a^{-1}
\end{array}\right), n(b)=\left(\begin{array}{ll}
1 & b \\
0 & 1
\end{array}\right), w=\left(\begin{array}{cc}
0 & 1 \\
-1 & 0
\end{array}\right),
$$

for $\phi \in \mathscr{S}\left(\mathbb{A}_{\mathbb{Q}}\right)$ a Schwartz-Bruhat function, the Weil representation $r$ for $\operatorname{SL}_{2}\left(\mathbb{A}_{\mathbb{Q}}\right)$ is defined by:

$-r(m(a)) \phi(x)=\chi_{0}(a)|a|^{1 / 2} \phi(a x)$

$-r(n(b)) \phi(x)=\psi\left(b x^{2}\right) \phi(x)$

$-r(w) \phi(x)=\gamma \widehat{\phi}(x)$,

where $\psi_{p}(x)=e^{-2 \pi i \operatorname{Frac}_{p}(x)}$ and $\psi_{\infty}(x)=e^{2 \pi i x}, \gamma$ is an 8th root of unity, and $\chi_{0}$ is a quadratic character. Here we choose the self-dual Haar measure such that $\widehat{\widehat{\phi}}(-x)=\phi(x)$. Note that this is the same Haar measure as chosen in Tate's thesis for the global field $\mathbb{Q}$ (see [1, Section 3.1]).

We define the Schwartz-Bruhat functions $\phi^{r, \mu}=\prod_{v} \phi_{v}^{r, \mu}$ for $\theta_{r, \mu}$ by taking $\phi_{\infty}^{r, \mu}(x)=e^{-2 \pi x^{2}}, \phi_{p}^{r, \mu}=\operatorname{char}_{\mathbb{Z}_{p}+\frac{r}{D}-\mu}$ for $p \neq 2$ and $\phi_{2}^{r, \mu}(x)=$ $e^{\pi i \operatorname{Frac}_{2}(x)} \operatorname{char}_{\mathbb{Z}_{2}-1 / 2}(x)$. Then for the theta function

$$
\theta\left(g, \phi^{r, \mu}\right)=\sum_{x \in \mathbb{Q}} r(g) \phi^{r, \mu}(x),
$$

for $g_{z}=\left(\begin{array}{cc}y^{1 / 2} & y^{-1 / 2} x \\ 0 & y^{-1 / 2}\end{array}\right)$, we can compute $\theta\left(g_{z}, \phi^{r, \mu}\right)=e^{-\pi i \operatorname{Frac}_{2} \mu}(-1)^{r} y^{1 / 4} \theta_{r, \mu}(2 z)$. For this type of standard computation see for example [10].

Using the properties of the Weil representation and the definition above for $\theta_{r, \mu}$, after a straightforward computation we get the following lemma that we use in the proof of Proposition 40, as well as below for Lemma 46 that is used in Sect. 5.2:

Lemma 45 For $\left(\begin{array}{ll}a & b \\ c & d\end{array}\right) \in \mathrm{SL}_{2}(\mathbb{Z})$ such that $3 D^{2} \mid b$ and $a \equiv 1 \bmod 6$, we have the transformation:

$$
\theta_{r, \mu}\left(\frac{a z+b}{c z+d}\right)=\operatorname{sgn}(d) e^{\pi i(a-1) / 2} \chi_{0,6}(a) e^{2 \pi i \operatorname{Frac}_{2}\left(\frac{b a-c / a}{8}\right)} e^{2 \pi i \operatorname{Frac}_{3}\left(t_{\mu} \frac{b a / 2}{9}\right)} \sqrt{c z+d} \theta_{a r, \mu}(z) .
$$

where $t_{1 / 2}=0$ and $t_{1 / 6}=1$.

Proof Let $\phi=\phi^{\mu, r}$. Recall that $\theta\left(g_{z / 2}, \phi\right)=e^{-\pi i \operatorname{Frac}_{2} \mu}(-1)^{r}(y / 2)^{1 / 2} \theta_{r, \mu}(z)$. We have

$$
\theta\left(m(\sqrt{2})^{-1}\left(\begin{array}{ll}
a & b \\
c & d
\end{array}\right) g_{z}, 1_{f}, \phi\right)=\theta\left(m(\sqrt{2})^{-1} g_{z},\left(\begin{array}{cc}
a & b / 2 \\
2 c & d
\end{array}\right)_{f}^{-1}, \phi\right)
$$

and we will compute separately the LHS and the RHS using the definition of the Weil representation. To compute the RHS, we rewrite the matrix $\left(\begin{array}{cc}d & -b / 2 \\ -2 c & a\end{array}\right)$ as $m\left(a^{-1}\right) n(-b a / 2) m(-1) w \cdot n(2 c / a) \cdot w$. 
At $p \nmid 6 D$, the action of $\left(\begin{array}{cc}d & -b / 2 \\ -2 c & a\end{array}\right)$ is trivial. For $p \mid D$, we can easily compute the Fourier transform $\widehat{\phi_{p}}(x)=e^{-2 \pi i \operatorname{Frac}_{p}(2 r x / D)} \operatorname{char}_{\mathbb{Z}_{p}}(x)$. Using $v_{p}(c / a) \geq 0$ and $v_{p}(b a / 2) \geq 2$, we get the action of $\left(\begin{array}{cc}d & -b / 2 \\ -2 c & a\end{array}\right)$ on $\phi_{p}$ to equal char $\mathbb{Z}_{p}+\frac{a r}{D}(x)$.

For $p=3$, the computation is similar. For $\mu=1 / 2$ we get $\chi_{0,3}(a) \phi_{3}(x)$, while for $\mu=1 / 6$ we get $\chi_{0,3}(a) e^{-2 \pi i \operatorname{Frac}_{3}\left(\frac{b a / 2}{9}\right)} \phi_{3}(x)$.

For $p=2$, we have the Fourier transform $\widehat{\phi_{2}}(x)=e^{\pi i / 2} e^{-2 \pi i \operatorname{Frac}_{2}(x)} \operatorname{char}_{\frac{1}{2}\left(\mathbb{Z}_{2}+1 / 2\right)}(x)$.

Using $v_{2}(2 c / a) \geq 1$ and $v_{2}(b a) \geq 0$ we get $\chi_{0,2}(a) e^{\pi i(a-1) / 2} e^{2 \pi i \operatorname{Frac}_{2}\left(\frac{b a-c / a}{8}\right)} \phi_{2}(x)$.

This finishes the computation, as we $\operatorname{got} \theta\left(m(\sqrt{2})^{-1} g_{z},\left(\begin{array}{cc}a & b / 2 \\ 2 c & d\end{array}\right)_{f}^{-1}, \phi\right)$ to equal:

$$
c_{0}(-1)^{r} e^{-\pi i \operatorname{Frac}_{2} \mu}(y / 2)^{1 / 4} \theta_{a r, \mu}(z)
$$

where $c_{0}=\chi_{0,6}(a) e^{\pi i(a-1) / 2} e^{2 \pi i \operatorname{Frac}_{2}\left(\frac{b a-c / a}{8}\right)} e^{2 \pi i t_{\mu} \operatorname{Frac}_{3}\left(\frac{b a / 2}{9}\right)}$.

For the LHS of (28) we have $r\left(g_{z}\right) \phi_{\infty}(m)=y^{1 / 4} e^{2 \pi i z m^{2}}$. Using the Weil representation action we get

$$
r\left(m(\sqrt{2})^{-1}\right) r\left(\begin{array}{ll}
a & b \\
c & d
\end{array}\right) r\left(g_{z}\right) \phi_{\infty}(x)=(y / 2)^{1 / 4} \operatorname{sgn}(d) \sqrt{\frac{1}{c z+d}} e^{\pi i\left(\frac{a z+b}{c z+d}\right) x^{2}},
$$

and thus combining with (29) we get the result of the lemma.

It follows immediately by applying the lemma above for $\theta_{r, \mu}$ and $\theta_{0}$ that:

Lemma $46 f_{r, 1 / 2}$ is a modular function for $\Gamma\left(18 D^{2}\right)$ and $f_{r, 1 / 6}$ is a modular function for $\Gamma\left(6 D^{2}\right)$.

Remark 47 Also from Lemma 45 it is easy to see that $f_{r, \mu}\left(z+9 D^{2}\right)=f_{r, \mu}(z)$.

We can also straightforwardly compute the transformation under $w=\left(\begin{array}{cc}0 & 1 \\ -1 & 0\end{array}\right)$ of $\theta_{r, \mu}$ and get the Lemma 48 below. This lemma is essential for the proof of Lemma 26.

Lemma 48 We have the transformation:

$$
\begin{aligned}
& \theta_{r, 1 / 6}(3 z) \\
& =(-1)^{r} \frac{\omega e^{\pi i \frac{D-1}{6}}}{\sqrt{-3} \sqrt{-i z}}\left(\theta^{(-3 r), 1 / 6}\left(\frac{-3}{z}\right)-\omega \theta^{(3 r), 1 / 6}\left(\frac{-3}{z}\right)-\omega^{2} \theta^{(-3 r), 1 / 2}\left(\frac{-3}{z}\right)\right),
\end{aligned}
$$

where $\theta^{(r), \mu}(z)=\sum_{n \in \mathbb{Z}} e^{\pi i(n-\mu)^{2} z}(-1)^{n} e^{2 \pi i n r / D}$.

Proof Denote $\phi=\phi^{r, 1 / 6}$. Then for $g_{Z / 2}=\left(\begin{array}{cc}(Y / 2)^{1 / 2} & (Y / 2)^{-1 / 2}(X / 2) \\ 0 & (Y / 2)^{-1 / 2}\end{array}\right)$ and $w=$ $\left(\begin{array}{cc}0 & 1 \\ -1 & 0\end{array}\right)$ we have $\theta\left(g_{Z / 2}, \phi\right)=\theta\left(w g_{Z / 2}, w \phi\right)$. We compute $\theta\left(w g_{Z / 2}, w \phi\right)$ below:

- At $\infty: r\left(w g_{Z / 2}\right) \phi_{\infty}(x)=\frac{\gamma_{\infty}(Y / 2)^{1 / 2} e^{4 \pi i x^{2}(-1 / Z)}}{\sqrt{-i Z}}$.

- At $p \mid D: r\left(w \phi_{p}\right)=\gamma_{p} e^{-2 \pi i \operatorname{Frac}_{p}(2 x r / D)} \operatorname{char}_{\mathbb{Z}_{p}}(x)$. 
- At $p=3: r\left(w \phi_{3}\right)=\gamma_{3} e^{-2 \pi i \operatorname{Frac}_{3}(2 x / 3)} \operatorname{char}_{\mathbb{Z}_{3}}(x)$.

- At $p=2: r\left(w \phi_{2}\right)=\gamma_{2} e^{-\pi i / 2} e^{2 \pi i \operatorname{Frac}_{2}(x)} \operatorname{char}_{\frac{1}{2}\left(\mathbb{Z}_{2}+1 / 2\right)}(x)$.

Writing all these together, we get:

$$
\theta\left(w g_{Z / 2}, w \phi\right)=\omega \frac{(Y / 2)^{1 / 4}}{\sqrt{-i Z}} \sum_{n \in \mathbb{Z}} e^{\pi i(n+1 / 2)^{2}(-1 / Z)}(-1)^{n} e^{-2 \pi i \operatorname{Frac}_{3}(n / 3)} e^{2 \pi i \operatorname{Frac}_{D}((n+1 / 2) r / D)}
$$

Changing $n \rightarrow-n-\frac{D-1}{2}$ we get on the RHS:

$e^{2 \pi i \frac{D-1}{3}}(-1)^{\frac{D-1}{2}} \omega \frac{(Y / 2)^{1 / 4}}{\sqrt{-i Z}} \sum_{n \in \mathbb{Z}} e^{\pi i(n-D / 2)^{2}(-1 / Z)}(-1)^{n} e^{2 \pi i \operatorname{Frac}_{3}(n / 3)} e^{-2 \pi i \operatorname{Frac}_{D}(n r / D)}$.

Taking the separate sums depending on $n \bmod 3$ we get a sum:

$$
\begin{aligned}
& \sum_{n \in \mathbb{Z}} e^{\pi i(n-D / 2)^{2}(-1 / Z)}(-1)^{n} e^{2 \pi i \operatorname{Frac}_{3}(n / 3)} e^{-2 \pi i \operatorname{Frac}_{D}(n r / D)} \\
& \quad=\theta^{(-3 r), 1 / 6}(9(-1 / Z))-\omega \theta^{(3 r), 1 / 6}(9(-1 / Z))-\omega^{2} \theta^{(-3 r), 1 / 2}(9(-1 / Z))
\end{aligned}
$$

Thus we got $e^{2 \pi i \frac{D-1}{3}}(-1)^{\frac{D-1}{2}} \omega \frac{(Y / 2)^{1 / 4}}{\sqrt{-i Z}}\left(\theta^{(-3 r), 1 / 6}\left(9\left(-\frac{1}{Z}\right)\right)-\omega \theta^{(3 r), 1 / 6}\left(9\left(-\frac{1}{Z}\right)\right)-\right.$ $\left.\omega^{2} \theta^{(-3 r), 1 / 2}\left(9\left(-\frac{1}{Z}\right)\right)\right)$. Taking $Z=3 z$ and using

$$
\theta\left(w g_{Z / 2}, w \phi\right)=\theta\left(g_{Z / 2}, \phi\right)=i(-1)^{r}(Y / 2)^{1 / 4} \theta_{r}(Z)
$$

we get the result of the lemma.

\section{References}

1. Bump, D.: Automorphic Forms and Representations, vol. 55. Cambridge University Press, Cambridge (1998)

2. Cassels, J.W.S.: Arithmetic on curves of genus 1, IV. Proof of the Hauptvermutung. J. Reine Angew. Math. 211, 95-112 (1962)

3. Coates, J., Wiles, A.: On the conjecture of Birch and Swinnerton-Dyer. Invent. Math. 39, 233-251 (1977)

4. Cohn, H.: Introduction to the Construction of Class Fields. Dover Publications, New York (1985)

5. Cox, D.A.: Primes of the form $x^{2}+n y^{2}$ : Fermat, class field theory, and complex multiplication. Wiley, New York (2011)

6. Dasgupta, S., Voight, J.: Heegner Points and Sylvester's Conjecture, Arithmetic Geometry (2009)

7. Gee, A.: Class fields by Shimura reciprocity. Universiteit van Amsterdam, PhD diss. (2001)

8. Gelbart, S.: Weil's Representation and the Spectrum of the Metaplectic Group, vol. 530. Springer, Berlin (2006)

9. Gross, B., Zagier, D.: Heegner points and derivatives of $L$-series. Invent. Math. 84(2), 225-320 (1986)

10. Hanke, J.: Quadratic Forms and Automorphic Forms. Quadratic and Higher Degree Forms, pp. 109168. Springer, New York (2013)

11. Hecke, E.: Mathematische Werke, Auftrage der Akademie der Wissenschaften zu Gottingen Vandenhoeck \& Ruprecht (1959) 
12. Hida, H.: Elliptic Curves and Arithmetic Invariants. Springer, New York (2013)

13. Ireland, K., Rosen, M.: A Classical Introduction to Modern Number Theory, vol. 84. Springer Science and Business Media, New York (2013)

14. Jetchev, D., Skinner, C., Wan, X.: The Birch and Swinnerton-Dyer formula for elliptic curves of analytic rank one (preprint) (2015). arXiv:1512.06894

15. Katz, N.M.: p-adic interpolation of real analytic Eisenstein series. Ann. Math. (2) 104(3), 459-571 (1976)

16. Köhler, G.: Eta Products and Theta Series Identities. Springer Science and Business Media, New York (2011)

17. Kolyvagin, V.A.: Finiteness of $E(\mathbb{Q})$ and $\amalg(E ; \mathbb{Q})$ for a subclass of Weil curves. Math. USSR Izvestiya 32(3) (1989)

18. Kowalski, E.: Automorphic forms, L-functions and number theory (March 12-16), Three introductory lectures

19. Lang, S.: Elliptic Functions. Springer, New York (1987)

20. Pacetti, A.: A formula for the central value of certain Hecke $L$-functions. J. Number Theory 113(2), 339-379 (2005)

21. Prasad, D.: A Brief Survey on the Theta Correspondence, Number Theory, January 3-6, 1996. Ramanujan Mathematical Society, Tiruchirapalli (1998)

22. Rodriguez-Villegas, F.: On the square root of special values of certain $L$-series. Invent. Math. 106(1), 549-573 (1991)

23. Rodriguez-Villegas, F., Zagier, D.: Square root of central values of Hecke $L$-functions. In: Gouvea, F.Q., Yui, N. (eds.) Advances in Number Theory, pp. 81-99. Clarendon Press, Oxford (1993)

24. Rodriguez-Villegas, F., Zagier, D.: Which primes are sums of two cubes?, Number theory (Halifax, NS, 1994), CMS Conf. Proc., vol. 15. American Mathematical Society, Providence, pp. 295-306 (1995)

25. Rubin, K.: The "main conjectures" of Iwasawa theory for imaginary quadratic fields. Invent. Math. 103, 25-68 (1991)

26. Selmer, E.S.: The diophantine equation $a x^{3}+b y^{3}+c z^{3}=0$. Acta Math. 85, 203-362 (1951)

27. Silverman, J.: The Arithmetic of Elliptic Curves, Graduate Texts in Mathematics, vol. 106. Springer, New York (1986)

28. Silverman, J.: Advanced Topics in the Arithmetic of Elliptic Curves, vol. 151. Springer Science and Business Media, New York (2013)

29. Shimura, G.: On the holomorphy of certain Dirichlet series. Proc. Lond. Math. Soc. 31(3), 79-98 (1975)

30. Stevenhagen, P.: Hilbert's 12th Problem, Complex Multiplication and Shimura Reciprocity. Department of Mathematics, Computer Science, Physics and Astronomy, University of Amsterdam, Amsterdam (1998)

31. Tunnell, J.B.: A classical diophantine problem and modular forms of weight $3 / 2$. Invent. Math. 72, 323-334 (1983)

32. Waldspurger, J.L.: Sur les coefficients de Fourier des formes modulaires de poids demi-entier. J. Math. Pures Appl. 60, 375-484 (1981)

33. Yuan, X., Zhang, S.-W., Zhang, W.: The Gross-Zagier Formula on Shimura Curves, vol. 184. Princeton University Press, Princeton (2013)

Publisher's Note Springer Nature remains neutral with regard to jurisdictional claims in published maps and institutional affiliations. 